\title{
Frozen Jacobian Multistep Iterative Method for Solving Nonlinear IVPs and BVPs
}

\author{
Fayyaz Ahmad, ${ }^{1,2,3}$ Shafiq Ur Rehman, ${ }^{4}$ Malik Zaka Ullah, ${ }^{2,5}$ \\ Hani Moaiteq Aljahdali, ${ }^{6}$ Shahid Ahmad, ${ }^{7}$ Ali Saleh Alshomrani, ${ }^{5}$ Juan A. Carrasco, ${ }^{8}$ \\ Shamshad Ahmad, ${ }^{9}$ and Sivanandam Sivasankaran ${ }^{10}$ \\ ${ }^{1}$ Departament de Física i Enginyeria Nuclear, Universitat Politècnica de Catalunya, Eduard Maristanu 10, \\ 08019 Barcelona, Spain \\ ${ }^{2}$ Dipartimento di Scienza e Alta Tecnologia, Universita dell'Insubria, Via Valleggio 11, 22100 Como, Italy \\ ${ }^{3}$ UCERD, Islamabad, Pakistan \\ ${ }^{4}$ Department of Mathematics, University of Engineering and Technology, Lahore, Pakistan \\ ${ }^{5}$ Department of Mathematics, King Abdulaziz University, Jeddah 21589, Saudi Arabia \\ ${ }^{6}$ Department of Information Systems, Faculty of Computing and Information Technology (Rabigh), King Abdulaziz University, \\ Rabigh 21911, Saudi Arabia \\ ${ }^{7}$ Department of Mathematics, Government College University Lahore, Lahore, Pakistan \\ ${ }^{8}$ Departament d'Enginyeria Electronica, Universitat Politècnica de Catalunya, Diagonal 647, Planta 9, 08028 Barcelona, Spain \\ ${ }^{9}$ Heat and Mass Transfer Technological Center, Universitat Politècnica de Catalunya, Colom 11, 08222 Terrassa, Spain \\ ${ }^{10}$ Institute of Mathematical Sciences, University of Malaya, Kuala Lumpur 50603, Malaysia
}

Correspondence should be addressed to Fayyaz Ahmad; fayyaz.ahmad@upc.edu

Received 30 July 2016; Revised 10 November 2016; Accepted 21 November 2016; Published 3 May 2017

Academic Editor: Alicia Cordero

Copyright (c) 2017 Fayyaz Ahmad et al. This is an open access article distributed under the Creative Commons Attribution License, which permits unrestricted use, distribution, and reproduction in any medium, provided the original work is properly cited.

In this paper, we present and illustrate a frozen Jacobian multistep iterative method to solve systems of nonlinear equations associated with initial value problems (IVPs) and boundary value problems (BVPs). We have used Jacobi-Gauss-Lobatto collocation (J-GL-C) methods to discretize the IVPs and BVPs. Frozen Jacobian multistep iterative methods are computationally very efficient. They require only one inversion of the Jacobian in the form of LU-factorization. The LU factors can then be used repeatedly in the multistep part to solve other linear systems. The convergence order of the proposed iterative method is $5 m-11$, where $m$ is the number of steps. The validity, accuracy, and efficiency of our proposed frozen Jacobian multistep iterative method is illustrated by solving fifteen IVPs and BVPs. It has been observed that, in all the test problems, with one exception in this paper, a single application of the proposed method is enough to obtain highly accurate numerical solutions. In addition, we present a comprehensive comparison of J-GL-C methods on a collection of test problems.

\section{Introduction}

Iterative methods for solving systems of nonlinear equations associated with IVPs and BVPs are important because, in general, it is hard to find a closed form solution. Generally, nonlinear IVPs and BVPs are solved in two steps. For the first step, we discretize the nonlinear problem to obtain a system of nonlinear equations. In the second step, we use some iterative method to solve the system of nonlinear equations.

Pseudospectral collocation methods offer excellent accuracy, and we use them for the discretization of IVPs and
BVPs. These methods convert the targeted partial or ordinary differential equations into a system of algebraic equations. Recently, Doha et al. [1] used a J-GL-C method for the discretization of a nonlinear 1+1 Schrödinger equation to obtain a system of ordinary differential equations and solved it by using an implicit Runge-Kutta method. Highly accurate results for four different kinds of nonlinear $1+1$ Schrödinger equations were obtained. In [2], nonlinear reaction-diffusion equations were solved by using a J-GL-C method. In [3], a J-GL-C method was also used to solve nonlinear complex generalized Zakharov systems of equations. Many authors 
(see, e.g., [4-7]) have used pseudospectral collocation method to solve nonlinear IVPs and BVPs. J-GL-C methods are attractive because they depend on two parameters and by changing the values of these parameters we can obtain different pseudospectral collocation methods, for example, the Legendre, Chebyshev, and Gegenbauer pseudospectral collocation methods. J-GL-C methods are based on Jacobi polynomials. The Jacobi polynomials are the eigenfunctions of the singular Sturm-Liouville problem [8],

$$
\begin{gathered}
\left(1-x^{2}\right) \vartheta^{\prime \prime}(x)+(\theta-\phi+(\theta+\phi+2) x) \vartheta^{\prime}(x) \\
+n(n+\theta+\phi+1) \vartheta(x)=0,
\end{gathered}
$$

and can be generated by using the following recurrence relation:

$$
\begin{aligned}
& J_{k+1}^{(\theta, \phi)}(x)=\left(a_{k}^{(\theta, \phi)}-b_{k}^{(\theta, \phi)}\right) J_{k}^{(\theta, \phi)}(x),-c_{k}^{(\theta, \phi)} J_{k-1}^{(\theta, \phi)}(x), \\
& k \geq 1, \\
& J_{0}^{(\theta, \phi)}(x)=1, \\
& J_{1}^{(\theta, \phi)}(x)=\frac{1}{2}(\theta+\phi+2) x+\frac{1}{2}(\phi-\theta),
\end{aligned}
$$

where

$$
\begin{aligned}
& a_{k}^{(\theta, \phi)}=\frac{(2 k+\theta+\phi+1)(2 k+\theta+\phi+2)}{2(k+1)(k+\theta+\phi+1)}, \\
& b_{k}^{(\theta, \phi)}=\frac{\left(\theta^{2}-\phi^{2}\right)(2 k+\theta+\phi+1)}{2(k+1)(k+\theta+\phi-1)(2 k+\theta+\phi)}, \\
& c_{k}^{(\theta, \phi)}=\frac{(k+\theta)(k+\phi)(2 k+\theta+\phi+2)}{(k+1)(k+\theta+\phi-1)(2 k+\theta+\phi)} .
\end{aligned}
$$

MNR
The $p$ th derivative of the $k$ th-degree Jacobi polynomial $J_{k}^{(\theta, \phi)}$ is given by

$$
D^{(p)} J_{k}^{(\theta, \phi)}(x)=\frac{\Gamma(j+\theta+\phi+p+1)}{2^{p} \Gamma(j+\theta+\phi+1)} J_{k-p}^{(\theta+p, \phi+p)}(x) .
$$

It should be noticed that Jacobi polynomials are orthogonal over the domain $[-1,1]$ with the weight function $(1+x)^{\theta}(1-$ $x)^{\phi}$. We are interested in approximating the differential operators by using J-GL-C methods. The easiest way to work with differential operators of different orders is to construct the differentiation matrix; for details on the construction of numerical differentiation matrix on Jacobi polynomials over the domain $[-1,1]$, see [9]. Suppose $Q$ is the Jacobi-GaussLobatto differentiation matrix for the first-order derivative operator over the domain $[-1,1]$. Then, a derivative of order $p$ can be approximated over the interval $[a, b]$ as

$$
\frac{d^{p}}{d x^{p}} \approx\left(\frac{2}{b-a} Q\right)^{p}
$$

After having a setup for the discretization of IVPs and BVPs by using J-GL-C methods, we are looking for an efficient iterative method for the solution of the associated system of nonlinear equations. The first iterative method that comes into mind to solve a system of nonlinear equations $\mathbf{F}(\mathbf{y})=\mathbf{0}$ is the Newton-Raphson (NR) method [10, 11], which can be written as

$$
\mathrm{NR}=\left\{\begin{array}{l}
\mathbf{y}_{0}=\text { initial guess } \\
\mathbf{y}_{n+1}=\mathbf{y}_{n}-\mathbf{F}^{\prime}\left(\mathbf{y}_{n}\right)^{-1} \mathbf{F}\left(\mathbf{y}_{n}\right),
\end{array}\right.
$$

where $\operatorname{det}\left(\mathbf{F}^{\prime}\left(\mathbf{y}_{n}\right)\right) \neq 0$. The multistep frozen Jacobian version of the Newton-Raphson method (MNR) can be written as

$$
=\left\{\begin{array}{l}
\text { Number of steps }=m \geq 1 \\
\text { Convergence order }=m+1 \\
\text { Function evaluations }=m \\
\text { Jacobian evaluations }=1 \\
\text { Number of LU-factorization }=1 \\
\text { Number of lower and upper triangular systems }=m
\end{array}\right.
$$

Frozen Jacobian methods have the advantage of computing LU decomposition of the Jacobian, because this makes the solution of linear systems, having the Jacobian as their matrix, inexpensive. However, the convergence order of MNR as a function of the number of function evaluations is relatively $\begin{aligned} & \left\{\begin{array}{l}\mathbf{F}^{\prime}\left(\mathbf{y}_{0}\right) \phi_{1}=\mathbf{F}\left(\mathbf{y}_{0}\right) \\ \mathbf{y}_{1}=\mathbf{y}_{0}-\phi_{1}\end{array}\right. \\ \text { Multi-step part } \longrightarrow & \left\{\begin{array}{r}\text { for } s=1, m-1 \\ \mathbf{F}^{\prime}\left(\mathbf{y}_{0}\right) \phi_{s+1}=\mathbf{F}\left(\mathbf{y}_{s}\right) \\ \mathbf{y}_{s+1}=\mathbf{y}_{s}-\phi_{s+1} \\ \text { end } \\ \mathbf{y}_{0}=\mathbf{y}_{m} .\end{array}\right.\end{aligned}$

low, and many researchers [12-23] have obtained frozen Jacobian multistep iterative methods with higher convergence orders for the same number of function evaluations. For example, HJ, FTUC, and MSF are presented in [22], [17], and [18], respectively. These methods can be describe as 
$\mathrm{HJ}=\left\{\begin{array}{l}\text { Number of steps }=m \geq 2 \\ \text { Convergence order }=2 m \\ \text { Function evaluations }=m-1 \\ \text { Jacobian evaluations }=2 \\ \mathrm{LU} \text {-factorization }=1 \\ \text { Matrix-vector multiplications }=m \\ \text { Vector-vector multiplications }=2 m \\ \text { Number of solutions of lower and upper triangular systems of equations }=2 m-1\end{array}\right.$

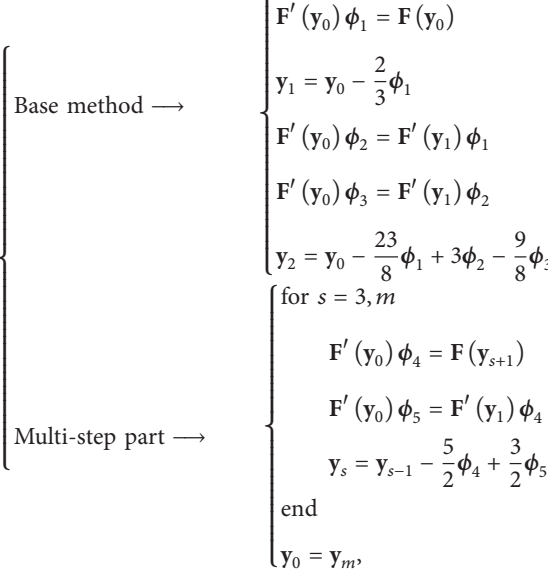

$\begin{cases}\text { Base method } \longrightarrow & \left\{\begin{array}{l}\mathbf{F}^{\prime}\left(\mathbf{y}_{0}\right) \phi_{1}=\mathbf{F}\left(\mathbf{y}_{0}\right) \\ \mathbf{y}_{1}=\mathbf{y}_{0}-\phi_{1} \\ \mathbf{F}^{\prime}\left(\mathbf{y}_{0}\right) \phi_{2}=\mathbf{F}\left(\mathbf{y}_{1}\right) \\ \mathbf{y}_{2}=\mathbf{y}_{1}-3 \phi_{2} \\ \mathbf{F}^{\prime}\left(\mathbf{y}_{0}\right) \phi_{3}=\mathbf{F}^{\prime}\left(\mathbf{y}_{2}\right) \phi_{2} \\ \mathbf{F}^{\prime}\left(\mathbf{y}_{0}\right) \phi_{4}=\mathbf{F}^{\prime}\left(\mathbf{y}_{2}\right) \phi_{3} \\ \mathbf{y}_{3}=\mathbf{y}_{1}-\frac{7}{4} \phi_{2}+\frac{1}{2} \phi_{3}+\frac{1}{4} \phi_{4}\end{array}\right. \\ \text { Multi-step part } \longrightarrow & \begin{array}{r}\text { for } s=4, m \\ \mathbf{F}^{\prime}\left(\mathbf{y}_{0}\right) \phi_{5}=\mathbf{F}\left(\mathbf{y}_{s}\right) \\ \mathbf{F}^{\prime}\left(\mathbf{y}_{0}\right) \phi_{6}=\mathbf{F}^{\prime}\left(\mathbf{y}_{2}\right) \phi_{5} \\ \mathbf{y}_{s}=\mathbf{y}_{s-1}-2 \phi_{5}+\phi_{6} \\ \text { end } \\ \mathbf{y}_{0}=\mathbf{y}_{m},\end{array}\end{cases}$

MSF
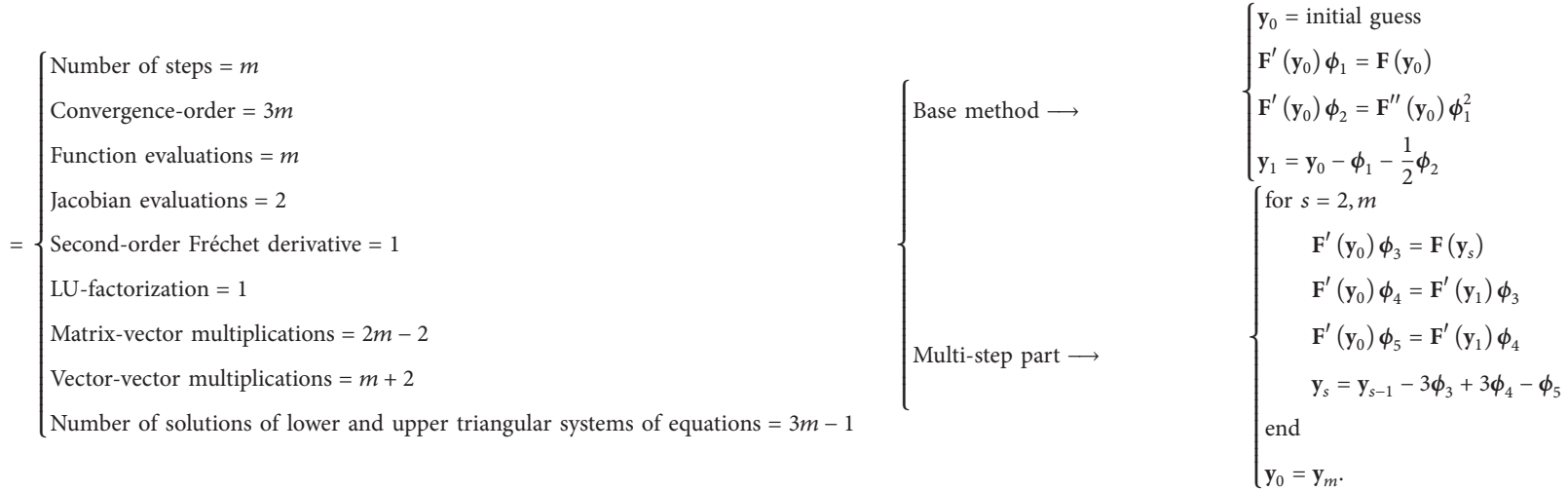

In this paper, we propose another frozen Jacobian multistep iterative method for solving systems of nonlinear equations with higher convergence order than the previously proposed frozen Jacobian methods for the same number of function evaluations. Another goal of the paper is to compare different pseudospectral collocation methods generated by JGL-C methods by choosing different values for the parameters $\theta$ and $\phi$. 


\section{New Multistep Iterative Method}

To construct the higher order frozen Jacobian multistep iterative method we first construct method with unknown coefficients as

$$
\text { for } i=1,3
$$

$$
\begin{aligned}
& \mathbf{F}^{\prime}\left(\mathbf{y}_{0}\right) \phi_{i}=\mathbf{F}\left(\mathbf{y}_{i-1}\right) \\
& \mathbf{y}_{i}=\mathbf{y}_{i-1}-\alpha_{i, 1} \phi_{i}
\end{aligned}
$$

end

$\mathbf{F}^{\prime}\left(\mathrm{y}_{0}\right) \phi_{4}=\mathbf{F}\left(\mathrm{y}_{3}\right)$

for $j=5, q$

$$
\mathbf{F}^{\prime}\left(\mathbf{y}_{0}\right) \phi_{j}=\mathbf{F}^{\prime}\left(\mathbf{y}_{3}\right) \phi_{j-1}
$$

end

$$
\begin{aligned}
& \mathbf{y}_{4}=\mathbf{y}_{3}+\sum_{k=1}^{q} \alpha_{4, k} \phi_{k} \\
& \mathbf{F}^{\prime}\left(\mathbf{y}_{0}\right) \phi_{q+1}=\mathbf{F}\left(\mathbf{y}_{4}\right) \\
& \text { for } j=q+2, r \\
& \mathbf{F}^{\prime}\left(\mathbf{y}_{0}\right) \phi_{j}=\mathbf{F}^{\prime}\left(\mathbf{y}_{3}\right) \phi_{j-1} \\
& \text { end } \\
& \mathbf{y}_{5}=\mathbf{y}_{4}+\sum_{k=q+1}^{r} \alpha_{5, k} \phi_{k} .
\end{aligned}
$$

NMIM
We have $r+3$ unknowns: namely, $\alpha_{1,1}, \alpha_{2,1}, \alpha_{3,1}, \alpha_{4,1}, \ldots$, $\alpha_{4, q}, \alpha_{5, q+1}, \ldots, \alpha_{5, r}$. To develop our method we find $q, r$, and these unknowns to obtain the maximum convergence order. Once we have solved that problem we write down the multistep part as

$$
\begin{aligned}
& \text { for } s=5, m \\
& \qquad \begin{array}{l}
\mathbf{F}^{\prime}\left(\mathbf{y}_{0}\right) \phi_{q+1}=\mathbf{F}\left(\mathbf{y}_{s-1}\right) \\
\text { for } j=q+2, r \\
\mathbf{F}^{\prime}\left(\mathbf{y}_{0}\right) \phi_{j}=\mathbf{F}^{\prime}\left(\mathbf{y}_{3}\right) \phi_{j-1}
\end{array}
\end{aligned}
$$

end

$$
\mathbf{y}_{s}=\mathbf{y}_{s-1}+\sum_{k=q+1}^{r} \alpha_{5, k} \phi_{k}
$$

end.

Notice that $\alpha_{5, q+1}, \ldots, \alpha_{5, r}$ are already computed in the base method part and remain fixed in the multistep part. After finding the values of unknowns, we establish our proposed new multistep iterative method (NMIM) as

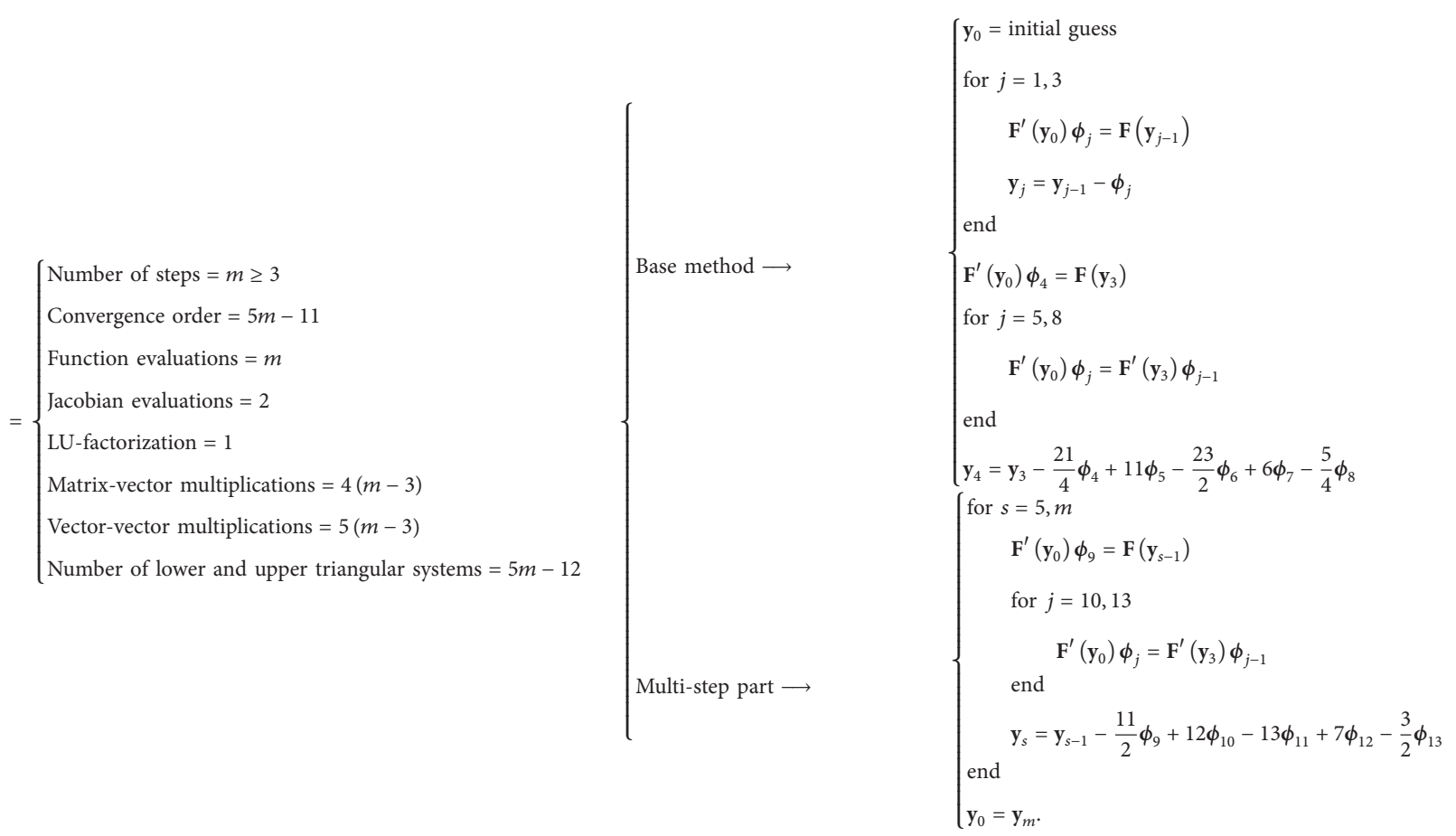

In NMIM, the convergence order of the base method is nine, and we get an increment of five in the convergence order per additional function evaluation. The LU factors of $\mathbf{F}^{\prime}\left(\mathbf{y}_{0}\right)$ are used in the multistep part to solve five lower and upper 
triangular systems of equations. NMIM requires only two Jacobian evaluations and the LU-factorization of one of the Jacobians.

\section{Convergence Analysis}

We use the method of mathematical induction to prove the convergence order of NMIM. We prove the convergence order for $m=4$ and, then, using induction, we will prove the convergence order for $m>4$. In our analysis, we expand the system of nonlinear equations around its simple root by using Taylor's series and use higher order Fréchet derivatives in the expansion. We use the symbolic algebra of Maple software to deal with symbolic computations. The Fréchet differentiability of the system of nonlinear equations is an essential constraint of our proof. Gateaux differentiability would not be enough, but that differentiability is neither enough to ensure that $\mathbf{F}(\mathbf{x})$ has a good local linear approximation, which is in soul of Newton-like methods. A function $\mathbf{F}(\cdot)$ is said to be Fréchet differentiable at a point $\mathbf{y}$ if there exists a linear operator $\mathbf{A} \in \mathbb{L}\left(\mathbb{R}^{n}, \mathbb{R}^{q}\right)$ such that

$$
\lim _{\mathbf{h} \rightarrow \mathbf{0}} \frac{\|\mathbf{F}(\mathbf{y}+\mathbf{h})-\mathbf{F}(\mathbf{y})-\mathbf{A h}\|}{\|\mathbf{h}\|}=0
$$

The linear operator $\mathbf{A}$ is called the first-order Fréchet derivative and we denote it by $\mathbf{F}^{\prime}(\mathbf{y})$. The higher order Fréchet derivatives can be computed recursively using

$$
\begin{aligned}
\mathbf{F}^{\prime}(\mathbf{y}) & =\operatorname{Jacobian}(\mathbf{F}(\mathbf{y})), \\
\mathbf{F}^{j}(\mathbf{y}) \mathbf{v}^{j-1} & =\operatorname{Jacobian}\left(\mathbf{F}^{j-1}(\mathbf{y}) \mathbf{v}^{j-1}\right), \quad j \geq 2,
\end{aligned}
$$

where $\mathbf{v}$ is a vector independent from $\mathbf{y}$.

Theorem 1. Let $\mathbf{F}: \Gamma \subseteq \mathbb{R}^{n} \rightarrow \mathbb{R}^{n}$ be a sufficiently Fréchet differentiable function on an open convex neighborhood $\Gamma$ of $\mathbf{y}^{*} \in \mathbb{R}^{n}$ with $\mathbf{F}\left(\mathbf{y}^{*}\right)=0$ and $\operatorname{det}\left(\mathbf{F}^{\prime}\left(\mathbf{y}^{*}\right)\right) \neq 0$, where $\mathbf{F}^{\prime}(\mathbf{y})$ denotes the Fréchet derivative of $\mathbf{F}(\mathbf{y})$. Let $\mathbf{A}_{1}=\mathbf{F}^{\prime}\left(\mathbf{y}^{*}\right)$ and $\mathbf{A}_{j}=(1 / j !) \mathbf{F}^{\prime}\left(\mathbf{y}^{*}\right)^{-1} \mathbf{F}^{(j)}\left(\mathbf{y}^{*}\right)$, for $j \geq 2$, where $\mathbf{F}^{(j)}(\mathbf{y})$ denotes the $j$-order Fréchet derivative of $\mathbf{F}(\mathbf{y})$. Then, for $m=4$, with an initial guess in the neighborhood of $\mathbf{y}^{*}$, the sequence $\left\{\mathbf{y}_{m}\right\}$ generated by NMIM converges to $\mathbf{y}^{*}$ with at least local order of convergence nine and error equation

$$
\mathbf{e}_{4}=\mathbf{L} \mathbf{e}_{0}{ }^{9}+O\left(\mathbf{e}_{0}{ }^{10}\right),
$$

where $\mathbf{e}_{0}=\mathbf{y}_{0}-\mathbf{y}^{*}, \mathbf{e}_{0}{ }^{p}=\overbrace{\left(\mathbf{e}_{0}, \mathbf{e}_{0}, \ldots, \mathbf{e}_{0}\right)}^{\text {ptimes }}$, and $\mathbf{L}=184 A_{2}^{8}+$ $8 A_{2}^{3} A_{3} A_{2}^{3}-24 A_{3} A_{2}^{6}$ is a 9-linear function: that is, $\mathbf{L} \in$ $\mathbb{L} \overbrace{\left(\mathbb{R}^{n}, \mathbb{R}^{n}, \mathbb{R}^{n}, \ldots, \mathbb{R}^{n}\right)}$ with $\mathbf{L e}_{0}{ }^{9} \in \mathbb{R}^{n}$.
Proof. We define the error at the $n$th step as $\mathbf{e}_{n}=\mathbf{y}_{n}-\mathbf{y}^{*}$. To complete the convergence proof, we perform detailed computations using Maple with the following results:

$$
\begin{aligned}
& \mathbf{F}\left(\mathbf{y}_{0}\right)=\mathbf{A}_{1}\left(\mathbf{e}_{0}+\mathbf{A}_{2} \mathbf{e}_{0}^{2}+\mathbf{A}_{3} \mathbf{e}_{0}^{3}+\mathbf{A}_{4} \mathbf{e}_{0}^{4}+\mathbf{A}_{5} \mathbf{e}_{0}^{5}+\mathbf{A}_{6} \mathbf{e}_{0}^{\mathbf{6}}\right. \\
& \left.+\mathbf{A}_{7} \mathbf{e}_{0}^{7}+\mathbf{A}_{8} \mathbf{e}_{0}^{8}+O\left(\mathbf{e}_{0}^{10}\right)\right), \\
& \mathbf{F}^{\prime}\left(\mathbf{y}_{0}\right)^{-1}=\left(\mathbf{I}-2 \mathbf{A}_{2} \mathbf{e}_{0}+\left(-3 \mathbf{A}_{3}+4 \mathbf{A}_{2}^{2}\right) \mathbf{e}_{0}^{2}+\left(-4 \mathbf{A}_{4}\right.\right. \\
& \left.+6 \mathbf{A}_{3} \mathbf{A}_{2}+6 \mathbf{A}_{2} \mathbf{A}_{3}-8 \mathbf{A}_{2}^{3}\right) \mathbf{e}_{0}^{3}+\left(-5 \mathbf{A}_{5}-12 \mathbf{A}_{2}^{2} \mathbf{A}_{3}\right. \\
& -12 \mathbf{A}_{3} \mathbf{A}_{2}^{2}-12 \mathbf{A}_{2} \mathbf{A}_{3} \mathbf{A}_{2}+8 \mathbf{A}_{4} \mathbf{A}_{2}+9 \mathbf{A}_{3}^{2}+8 \mathbf{A}_{2} \mathbf{A}_{4} \\
& \left.+16 \mathbf{A}_{2}^{4}\right) \mathbf{e}_{0}^{4}+\left(-6 \mathbf{A}_{6}-16 \mathbf{A}_{2}^{2} \mathbf{A}_{4}-32 \mathbf{A}_{2}^{5}-16 \mathbf{A}_{4} \mathbf{A}_{2}^{2}\right. \\
& -18 \mathbf{A}_{3}^{2} \mathbf{A}_{2}-16 \mathbf{A}_{2} \mathbf{A}_{4} \mathbf{A}_{2}-18 \mathbf{A}_{3} \mathbf{A}_{2} \mathbf{A}_{3}-18 \mathbf{A}_{2} \mathbf{A}_{3}^{2} \\
& +24 \mathbf{A}_{3} \mathbf{A}_{2}^{3}+24 \mathbf{A}_{2} \mathbf{A}_{3} \mathbf{A}_{2}^{2}+24 \mathbf{A}_{2}^{2} \mathbf{A}_{3} \mathbf{A}_{2}+10 \mathbf{A}_{5} \mathbf{A}_{2} \\
& \left.+12 \mathbf{A}_{4} \mathbf{A}_{3}+12 \mathbf{A}_{3} \mathbf{A}_{4}+10 \mathbf{A}_{2} \mathbf{A}_{5}+24 \mathbf{A}_{2}^{3} \mathbf{A}_{3}\right) \mathbf{e}_{0}^{5}+\cdots \\
& \left.+O\left(\mathbf{e}_{0}^{10}\right)\right) \mathbf{A}_{1}^{-1}, \\
& \phi_{1}=\mathbf{e}_{0}-\mathbf{A}_{2} \mathbf{e}_{0}^{2}+\left(-2 \mathbf{A}_{3}+2 \mathbf{A}_{2}^{2}\right) \mathbf{e}_{0}^{3}+\left(-3 \mathbf{A}_{4}+4 \mathbf{A}_{2} \mathbf{A}_{3}\right. \\
& \left.+3 \mathbf{A}_{3} \mathbf{A}_{2}-4 \mathbf{A}_{2}^{3}\right) \mathbf{e}_{0}^{4}+\left(-4 \mathbf{A}_{5}+6 \mathbf{A}_{2} \mathbf{A}_{4}+4 \mathbf{A}_{4} \mathbf{A}_{2}\right. \\
& \left.-6 \mathbf{A}_{2} \mathbf{A}_{3} \mathbf{A}_{2}+6 \mathbf{A}_{3}^{2}+8 \mathbf{A}_{2}^{4}-6 \mathbf{A}_{3} \mathbf{A}_{2}^{2}-8 \mathbf{A}_{2}^{2} \mathbf{A}_{3}\right) \mathbf{e}_{0}^{5} \\
& +\left(-5 \mathbf{A}_{6}-12 \mathbf{A}_{3} \mathbf{A}_{2} \mathbf{A}_{3}-8 \mathbf{A}_{2} \mathbf{A}_{4} \mathbf{A}_{2}+8 \mathbf{A}_{2} \mathbf{A}_{5}\right. \\
& +9 \mathbf{A}_{3} \mathbf{A}_{4}+8 \mathbf{A}_{4} \mathbf{A}_{3}+5 \mathbf{A}_{5} \mathbf{A}_{2}+12 \mathbf{A}_{2}^{2} \mathbf{A}_{3} \mathbf{A}_{2} \\
& +12 \mathbf{A}_{2} \mathbf{A}_{3} \mathbf{A}_{2}^{2}+12 \mathbf{A}_{3} \mathbf{A}_{2}^{3}-9 \mathbf{A}_{3}^{2} \mathbf{A}_{2}-12 \mathbf{A}_{2} \mathbf{A}_{3}^{2} \\
& \left.-16 \mathbf{A}_{2}^{5}-12 \mathbf{A}_{2}^{2} \mathbf{A}_{4}-8 \mathbf{A}_{4} \mathbf{A}_{2}^{2}+16 \mathbf{A}_{2}^{3} \mathbf{A}_{3}\right) \mathbf{e}_{0}^{6}+\cdots \\
& +O\left(\mathbf{e}_{0}^{10}\right) \text {, } \\
& \mathbf{e}_{1}=\mathbf{A}_{2} \mathbf{e}_{0}^{2}+\left(2 \mathbf{A}_{3}-2 \mathbf{A}_{2}^{2}\right) \mathbf{e}_{0}^{3}+\left(3 \mathbf{A}_{4}-4 \mathbf{A}_{2} \mathbf{A}_{3}\right. \\
& \left.-3 \mathbf{A}_{3} \mathbf{A}_{2}+4 \mathbf{A}_{2}^{3}\right) \mathbf{e}_{0}^{4}+\left(4 \mathbf{A}_{5}-6 \mathbf{A}_{2} \mathbf{A}_{4}-4 \mathbf{A}_{4} \mathbf{A}_{2}\right. \\
& \left.+6 \mathbf{A}_{2} \mathbf{A}_{3} \mathbf{A}_{2}-6 \mathbf{A}_{3}^{2}-8 \mathbf{A}_{2}^{4}+6 \mathbf{A}_{3} \mathbf{A}_{2}^{2}+8 \mathbf{A}_{2}^{2} \mathbf{A}_{3}\right) \mathbf{e}_{0}^{5} \\
& +\left(5 \mathbf{A}_{6}+12 \mathbf{A}_{3} \mathbf{A}_{2} \mathbf{A}_{3}+8 \mathbf{A}_{2} \mathbf{A}_{4} \mathbf{A}_{2}-8 \mathbf{A}_{2} \mathbf{A}_{5}\right. \\
& -9 \mathbf{A}_{3} \mathbf{A}_{4}-8 \mathbf{A}_{4} \mathbf{A}_{3}-5 \mathbf{A}_{5} \mathbf{A}_{2}-12 \mathbf{A}_{2}^{2} \mathbf{A}_{3} \mathbf{A}_{2} \\
& -12 \mathbf{A}_{2} \mathbf{A}_{3} \mathbf{A}_{2}^{2}-12 \mathbf{A}_{3} \mathbf{A}_{2}^{3}+9 \mathbf{A}_{3}^{2} \mathbf{A}_{2}+12 \mathbf{A}_{2} \mathbf{A}_{3}^{2} \\
& \left.+16 \mathbf{A}_{2}^{5}+12 \mathbf{A}_{2}^{2} \mathbf{A}_{4}+8 \mathbf{A}_{4} \mathbf{A}_{2}^{2}-16 \mathbf{A}_{2}^{3} \mathbf{A}_{3}\right) \mathbf{e}_{0}^{6}+\cdots \\
& +O\left(\mathbf{e}_{0}^{10}\right), \\
& \mathbf{F}\left(\mathbf{y}_{1}\right)=\mathbf{A}_{1}\left(\mathbf{A}_{2} \mathbf{e}_{0}^{2}+\left(2 \mathbf{A}_{3}-2 \mathbf{A}_{2}^{2}\right) \mathbf{e}_{0}^{3}+\left(3 \mathbf{A}_{4}-4 \mathbf{A}_{2} \mathbf{A}_{3}\right.\right. \\
& \left.-3 \mathbf{A}_{3} \mathbf{A}_{2}+5 \mathbf{A}_{2}^{3}\right) \mathbf{e}_{0}^{4}+\left(4 \mathbf{A}_{5}-6 \mathbf{A}_{2} \mathbf{A}_{4}-6 \mathbf{A}_{3}^{2}\right. \\
& \left.-4 \mathbf{A}_{4} \mathbf{A}_{2}-12 \mathbf{A}_{2}^{4}+8 \mathbf{A}_{2} \mathbf{A}_{3} \mathbf{A}_{2}+6 \mathbf{A}_{3} \mathbf{A}_{2}^{2}+10 \mathbf{A}_{2}^{2} \mathbf{A}_{3}\right)
\end{aligned}
$$




$$
\begin{aligned}
& -\mathbf{e}_{0}^{5}+\left(5 \mathbf{A}_{6}+8 \mathbf{A}_{4} \mathbf{A}_{2}^{2}+12 \mathbf{A}_{3} \mathbf{A}_{2} \mathbf{A}_{3}-24 \mathbf{A}_{2}^{3} \mathbf{A}_{3}\right. \\
& -19 \mathbf{A}_{2}^{2} \mathbf{A}_{3} \mathbf{A}_{2}-19 \mathbf{A}_{2} \mathbf{A}_{3} \mathbf{A}_{2}^{2}-11 \mathbf{A}_{3} \mathbf{A}_{2}^{3}+9 \mathbf{A}_{3}^{2} \mathbf{A}_{2} \\
& +11 \mathbf{A}_{2} \mathbf{A}_{4} \mathbf{A}_{2}+16 \mathbf{A}_{2} \mathbf{A}_{3}^{2}+28 \mathbf{A}_{2}^{5}-8 \mathbf{A}_{2} \mathbf{A}_{5}-9 \mathbf{A}_{3} \mathbf{A}_{4} \\
& \left.\left.-8 \mathbf{A}_{4} \mathbf{A}_{3}-5 \mathbf{A}_{5} \mathbf{A}_{2}+15 \mathbf{A}_{2}^{2} \mathbf{A}_{4}\right) \mathbf{e}_{0}^{6}+\cdots+O\left(\mathbf{e}_{0}^{10}\right)\right) \\
\boldsymbol{\phi}_{2} & =\mathbf{A}_{2} \mathbf{e}_{0}^{2}+\left(-4 \mathbf{A}_{2}^{2}+2 \mathbf{A}_{3}\right) \mathbf{e}_{0}^{3}+\left(3 \mathbf{A}_{4}+13 \mathbf{A}_{2}^{3}\right. \\
& \left.-8 \mathbf{A}_{2} \mathbf{A}_{3}-6 \mathbf{A}_{3} \mathbf{A}_{2}\right) \mathbf{e}_{0}^{4}+\left(4 \mathbf{A}_{5}-12 \mathbf{A}_{2} \mathbf{A}_{4}-12 \mathbf{A}_{3}^{2}\right. \\
& -8 \mathbf{A}_{4} \mathbf{A}_{2}-38 \mathbf{A}_{2}^{4}+20 \mathbf{A}_{2} \mathbf{A}_{3} \mathbf{A}_{2}+18 \mathbf{A}_{3} \mathbf{A}_{2}^{2} \\
& \left.+26 \mathbf{A}_{2}^{2} \mathbf{A}_{3}\right) \mathbf{e}_{0}^{5}+\left(5 \mathbf{A}_{6}+24 \mathbf{A}_{4} \mathbf{A}_{2}^{2}+36 \mathbf{A}_{3} \mathbf{A}_{2} \mathbf{A}_{3}\right. \\
& -76 \mathbf{A}_{2}^{3} \mathbf{A}_{3}-59 \mathbf{A}_{2}^{2} \mathbf{A}_{3} \mathbf{A}_{2}-55 \mathbf{A}_{2} \mathbf{A}_{3} \mathbf{A}_{2}^{2}-50 \mathbf{A}_{3} \mathbf{A}_{2}^{3} \\
& +27 \mathbf{A}_{3}^{2} \mathbf{A}_{2}+27 \mathbf{A}_{2} \mathbf{A}_{4} \mathbf{A}_{2}+40 \mathbf{A}_{2} \mathbf{A}_{3}^{2}+104 \mathbf{A}_{2}^{5} \\
& +16 \mathbf{A}_{2} \mathbf{A}_{5}-18 \mathbf{A}_{3} \mathbf{A}_{4}-16 \mathbf{A}_{4} \mathbf{A}_{3}-10 \mathbf{A}_{5} \mathbf{A}_{2} \\
& \left.+8 \mathbf{A}_{2} \mathbf{A}_{5}+9 \mathbf{A}_{3} \mathbf{A}_{4}+8 \mathbf{A}_{4} \mathbf{A}_{3}+5 \mathbf{A}_{5} \mathbf{A}_{2}-27 \mathbf{A}_{2}^{2} \mathbf{A}_{4}\right) \\
& \left.+39 \mathbf{A}_{2}^{2} \mathbf{A}_{4}\right) \mathbf{e}_{0}^{6}+\cdots+O\left(\mathbf{e}_{0}^{10}\right) \\
& \left.+18 \mathbf{A}_{2}^{2} \mathbf{A}_{3}\right) \mathbf{e}_{0}^{5}+\left(47 \mathbf{A}_{2}^{2} \mathbf{A}_{3} \mathbf{A}_{2}+43 \mathbf{A}_{2}^{3}-16 \mathbf{A}_{4} \mathbf{A}_{2}^{2}-18 \mathbf{A}_{3}^{2} \mathbf{A}_{2}^{2}-19 \mathbf{A}_{2} \mathbf{A}_{4} \mathbf{A}_{2}\right. \\
& +2 \mathbf{A}_{3} \mathbf{A}_{2} \mathbf{A}_{3}-28 \mathbf{A}_{2} \mathbf{A}_{3}^{2}+60 \mathbf{A}_{2}^{3} \mathbf{A}_{3}-88 \mathbf{A}_{2}^{5} \\
& =2 \mathbf{e}_{0}^{3} \mathbf{A}_{2}^{2}+\left(-9 \mathbf{A}_{2}^{3}+4 \mathbf{A}_{2} \mathbf{A}_{3}+3 \mathbf{A}_{3} \mathbf{A}_{2}\right) \mathbf{e}_{0}^{4}+\left(30 \mathbf{A}_{2}^{4}\right. \\
& +6 \mathbf{A}_{3}^{2}+4 \mathbf{A}_{4} \mathbf{A}_{2}-14 \mathbf{A}_{2} \mathbf{A}_{3} \mathbf{A}_{2}-12 \mathbf{A}_{3} \mathbf{A}_{2}^{2} \\
& +3 \mathbf{A}_{2}
\end{aligned}
$$$$
\mathbf{F}\left(\mathbf{y}_{2}\right)=\mathbf{A}_{1}\left(2 \mathbf{A}_{2}^{2} \mathbf{e}_{0}^{3}+\left(-9 \mathbf{A}_{2}^{3}+4 \mathbf{A}_{2} \mathbf{A}_{3}+3 \mathbf{A}_{3} \mathbf{A}_{2}\right) \mathbf{e}_{0}^{4}\right.
$$$$
+\left(30 \mathbf{A}_{2}^{4}+6 \mathbf{A}_{2} \mathbf{A}_{4}+6 \mathbf{A}_{3}^{2}+4 \mathbf{A}_{4} \mathbf{A}_{2}-14 \mathbf{A}_{2} \mathbf{A}_{3} \mathbf{A}_{2}\right.
$$$$
\left.-12 \mathbf{A}_{3} \mathbf{A}_{2}^{2}-18 \mathbf{A}_{2}^{2} \mathbf{A}_{3}\right) \mathbf{e}_{0}^{5}+\left(47 \mathbf{A}_{2}^{2} \mathbf{A}_{3} \mathbf{A}_{2}\right.
$$$$
+43 \mathbf{A}_{2} \mathbf{A}_{3} \mathbf{A}_{2}^{2}+38 \mathbf{A}_{3} \mathbf{A}_{2}^{3}-16 \mathbf{A}_{4} \mathbf{A}_{2}^{2}-18 \mathbf{A}_{3}^{2} \mathbf{A}_{2}
$$$$
-19 \mathbf{A}_{2} \mathbf{A}_{4} \mathbf{A}_{2}-24 \mathbf{A}_{3} \mathbf{A}_{2} \mathbf{A}_{3}-28 \mathbf{A}_{2} \mathbf{A}_{3}^{2}+60 \mathbf{A}_{2}^{3} \mathbf{A}_{3}
$$$$
-84 \mathbf{A}_{2}^{5}+8 \mathbf{A}_{2} \mathbf{A}_{5}+9 \mathbf{A}_{3} \mathbf{A}_{4}+8 \mathbf{A}_{4} \mathbf{A}_{3}+5 \mathbf{A}_{5} \mathbf{A}_{2}
$$$$
\left.\left.-27 \mathbf{A}_{2}^{2} \mathbf{A}_{4}\right) \mathbf{e}_{0}^{6}+\cdots+O\left(\mathbf{e}_{0}^{10}\right)\right)
$$$$
\phi_{3}=2 \mathbf{A}_{2}^{2} \mathbf{e}_{0}^{3}+\left(-13 \mathbf{A}_{2}^{3}+4 \mathbf{A}_{2} \mathbf{A}_{3}+3 \mathbf{A}_{3} \mathbf{A}_{2}\right) \mathbf{e}_{0}^{4}
$$$$
+\left(6 \mathbf{A}_{2} \mathbf{A}_{4}+4 \mathbf{A}_{4} \mathbf{A}_{2}-20 \mathbf{A}_{2} \mathbf{A}_{3} \mathbf{A}_{2}+56 \mathbf{A}_{2}^{4}+6 \mathbf{A}_{3}^{2}\right.
$$$$
\left.-18 \mathbf{A}_{3} \mathbf{A}_{2}^{2}-26 \mathbf{A}_{2}^{2} \mathbf{A}_{3}\right) \mathbf{e}_{0}^{5}+\left(-27 \mathbf{A}_{2} \mathbf{A}_{4} \mathbf{A}_{2}\right.
$$$$
-36 \mathbf{A}_{3} \mathbf{A}_{2} \mathbf{A}_{3}+8 \mathbf{A}_{2} \mathbf{A}_{5}+9 \mathbf{A}_{3} \mathbf{A}_{4}+8 \mathbf{A}_{4} \mathbf{A}_{3}+5 \mathbf{A}_{5} \mathbf{A}_{2}
$$$$
+87 \mathbf{A}_{2}^{2} \mathbf{A}_{3} \mathbf{A}_{2}+79 \mathbf{A}_{2} \mathbf{A}_{3} \mathbf{A}_{2}^{2}+77 \mathbf{A}_{3} \mathbf{A}_{2}^{3}-24 \mathbf{A}_{4} \mathbf{A}_{2}^{2}
$$

$$
\begin{aligned}
& -27 \mathbf{A}_{3}^{2} \mathbf{A}_{2}-40 \mathbf{A}_{2} \mathbf{A}_{3}^{2}+112 \mathbf{A}_{2}^{3} \mathbf{A}_{3}-196 \mathbf{A}_{2}^{5} \\
& \left.-39 \mathbf{A}_{2}^{2} \mathbf{A}_{4}\right) \mathbf{e}_{0}^{6}+\cdots+O\left(\mathbf{e}_{0}^{10}\right) \\
\mathbf{e}_{3} & =4 \mathbf{A}_{2}^{3} \mathbf{e}_{0}^{4}+\left(-26 \mathbf{A}_{2}^{4}+6 \mathbf{A}_{2} \mathbf{A}_{3} \mathbf{A}_{2}+6 \mathbf{A}_{3} \mathbf{A}_{2}^{2}\right. \\
& \left.+8 \mathbf{A}_{2}^{2} \mathbf{A}_{3}\right) \mathbf{e}_{0}^{5}+\left(-40 \mathbf{A}_{2}^{2} \mathbf{A}_{3} \mathbf{A}_{2}-36 \mathbf{A}_{2} \mathbf{A}_{3} \mathbf{A}_{2}^{2}\right. \\
& -39 \mathbf{A}_{3} \mathbf{A}_{2}^{3}+8 \mathbf{A}_{4} \mathbf{A}_{2}^{2}+9 \mathbf{A}_{3}^{2} \mathbf{A}_{2}+8 \mathbf{A}_{2} \mathbf{A}_{4} \mathbf{A}_{2} \\
& +12 \mathbf{A}_{3} \mathbf{A}_{2} \mathbf{A}_{3}+12 \mathbf{A}_{2} \mathbf{A}_{3}^{2}-52 \mathbf{A}_{2}^{3} \mathbf{A}_{3}+108 \mathbf{A}_{2}^{5} \\
& \left.+12 \mathbf{A}_{2}^{2} \mathbf{A}_{4}\right) \mathbf{e}_{0}^{6}+\left(-80 \mathbf{A}_{2}^{2} \mathbf{A}_{3}^{2}-72 \mathbf{A}_{2} \mathbf{A}_{3} \mathbf{A}_{2} \mathbf{A}_{3}\right. \\
& -78 \mathbf{A}_{3} \mathbf{A}_{2}^{2} \mathbf{A}_{3}+16 \mathbf{A}_{4} \mathbf{A}_{2} \mathbf{A}_{3}+18 \mathbf{A}_{3}^{3}+16 \mathbf{A}_{2} \mathbf{A}_{4} \mathbf{A}_{3} \\
& +18 \mathbf{A}_{2} \mathbf{A}_{3} \mathbf{A}_{4}+18 \mathbf{A}_{3} \mathbf{A}_{2} \mathbf{A}_{4}+216 \mathbf{A}_{2}^{4} \mathbf{A}_{3}-78 \mathbf{A}_{2}^{3} \mathbf{A}_{4} \\
& -356 \mathbf{A}_{2}^{6}+10 \mathbf{A}_{5} \mathbf{A}_{2}^{2}+12 \mathbf{A}_{4} \mathbf{A}_{3} \mathbf{A}_{2}+12 \mathbf{A}_{3} \mathbf{A}_{4} \mathbf{A}_{2} \\
& +10 \mathbf{A}_{2} \mathbf{A}_{5} \mathbf{A}_{2}+168 \mathbf{A}_{2}^{3} \mathbf{A}_{3} \mathbf{A}_{2}+150 \mathbf{A}_{2}^{2} \mathbf{A}_{3} \mathbf{A}_{2}^{2} \\
+ & 148 \mathbf{A}_{2} \mathbf{A}_{3} \mathbf{A}_{2}^{3}+168 \mathbf{A}_{3} \mathbf{A}_{2}^{4}+16 \mathbf{A}_{2}^{2} \mathbf{A}_{5}-52 \mathbf{A}_{4} \mathbf{A}_{2}^{3} \\
& -54 \mathbf{A}_{3}^{2} \mathbf{A}_{2}^{2}-48 \mathbf{A}_{2} \mathbf{A}_{4} \mathbf{A}_{2}^{2}-54 \mathbf{A}_{2} \mathbf{A}_{3}^{2} \mathbf{A}_{2} \\
& \left.-60 \mathbf{A}_{3} \mathbf{A}_{2} \mathbf{A}_{3} \mathbf{A}_{2}-54 \mathbf{A}_{2}^{2} \mathbf{A}_{4} \mathbf{A}_{2}\right) \mathbf{e}_{0}^{7}+\cdots+O\left(\mathbf{e}_{0}^{10}\right)
\end{aligned}
$$$$
\mathbf{F}\left(\mathbf{y}_{3}\right)=\mathbf{A}_{1}\left(4 \mathbf{A}_{2}^{3} \mathbf{e}_{0}^{4}+\left(-26 \mathbf{A}_{2}^{4}+6 \mathbf{A}_{2} \mathbf{A}_{3} \mathbf{A}_{2}+6 \mathbf{A}_{3} \mathbf{A}_{2}^{2}\right.\right.
$$$$
\left.+8 \mathbf{A}_{2}^{2} \mathbf{A}_{3}\right) \mathbf{e}_{0}^{5}+\left(-40 \mathbf{A}_{2}^{2} \mathbf{A}_{3} \mathbf{A}_{2}-36 \mathbf{A}_{2} \mathbf{A}_{3} \mathbf{A}_{2}^{2}\right.
$$$$
-39 \mathbf{A}_{3} \mathbf{A}_{2}^{3}+8 \mathbf{A}_{4} \mathbf{A}_{2}^{2}+9 \mathbf{A}_{3}^{2} \mathbf{A}_{2}+8 \mathbf{A}_{2} \mathbf{A}_{4} \mathbf{A}_{2}
$$$$
+12 \mathbf{A}_{3} \mathbf{A}_{2} \mathbf{A}_{3}+12 \mathbf{A}_{2} \mathbf{A}_{3}^{2}-52 \mathbf{A}_{2}^{3} \mathbf{A}_{3}+108 \mathbf{A}_{2}^{5}
$$$$
\left.+12 \mathbf{A}_{2}^{2} \mathbf{A}_{4}\right) \mathbf{e}_{0}^{6}+\left(-80 \mathbf{A}_{2}^{2} \mathbf{A}_{3}^{2}-72 \mathbf{A}_{2} \mathbf{A}_{3} \mathbf{A}_{2} \mathbf{A}_{3}\right.
$$$$
-78 \mathbf{A}_{3} \mathbf{A}_{2}^{2} \mathbf{A}_{3}+16 \mathbf{A}_{4} \mathbf{A}_{2} \mathbf{A}_{3}+18 \mathbf{A}_{3}^{3}+16 \mathbf{A}_{2} \mathbf{A}_{4} \mathbf{A}_{3}
$$$$
+18 \mathbf{A}_{2} \mathbf{A}_{3} \mathbf{A}_{4}+18 \mathbf{A}_{3} \mathbf{A}_{2} \mathbf{A}_{4}+216 \mathbf{A}_{2}^{4} \mathbf{A}_{3}-78 \mathbf{A}_{2}^{3} \mathbf{A}_{4}
$$$$
-356 \mathbf{A}_{2}^{6}+10 \mathbf{A}_{5} \mathbf{A}_{2}^{2}+12 \mathbf{A}_{4} \mathbf{A}_{3} \mathbf{A}_{2}+12 \mathbf{A}_{3} \mathbf{A}_{4} \mathbf{A}_{2}
$$$$
+10 \mathbf{A}_{2} \mathbf{A}_{5} \mathbf{A}_{2}+168 \mathbf{A}_{2}^{3} \mathbf{A}_{3} \mathbf{A}_{2}+150 \mathbf{A}_{2}^{2} \mathbf{A}_{3} \mathbf{A}_{2}^{2}
$$$$
+148 \mathbf{A}_{2} \mathbf{A}_{3} \mathbf{A}_{2}^{3}+168 \mathbf{A}_{3} \mathbf{A}_{2}^{4}+16 \mathbf{A}_{2}^{2} \mathbf{A}_{5}-52 \mathbf{A}_{4} \mathbf{A}_{2}^{3}
$$$$
-54 \mathbf{A}_{3}^{2} \mathbf{A}_{2}^{2}-48 \mathbf{A}_{2} \mathbf{A}_{4} \mathbf{A}_{2}^{2}-54 \mathbf{A}_{2} \mathbf{A}_{3}^{2} \mathbf{A}_{2}
$$$$
\left.\left.-60 \mathbf{A}_{3} \mathbf{A}_{2} \mathbf{A}_{3} \mathbf{A}_{2}-54 \mathbf{A}_{2}^{2} \mathbf{A}_{4} \mathbf{A}_{2}\right) \mathbf{e}_{0}^{7}+\cdots+O\left(\mathbf{e}_{0}^{10}\right)\right) \text {, }
$$

$\mathbf{F}^{\prime}\left(\mathbf{y}_{3}\right)=\mathbf{A}_{1}\left(\mathbf{I}+8 \mathbf{A}_{2}^{4} \mathbf{e}_{0}^{4}+\left(12 \mathbf{A}_{2} \mathbf{A}_{3} \mathbf{A}_{2}^{2}+12 \mathbf{A}_{2}^{2} \mathbf{A}_{3} \mathbf{A}_{2}\right.\right.$

$\left.-52 \mathbf{A}_{2}^{5}+16 \mathbf{A}_{2}^{3} \mathbf{A}_{3}\right) \mathbf{e}_{0}^{5}+\left(24 \mathbf{A}_{2}^{2} \mathbf{A}_{3}^{2}+24 \mathbf{A}_{2} \mathbf{A}_{3} \mathbf{A}_{2} \mathbf{A}_{3}\right.$

$-80 \mathbf{A}_{2}^{3} \mathbf{A}_{3} \mathbf{A}_{2}-72 \mathbf{A}_{2}^{2} \mathbf{A}_{3} \mathbf{A}_{2}^{2}-78 \mathbf{A}_{2} \mathbf{A}_{3} \mathbf{A}_{2}^{3}$

$+16 \mathbf{A}_{2} \mathbf{A}_{4} \mathbf{A}_{2}^{2}+18 \mathbf{A}_{2} \mathbf{A}_{3}^{2} \mathbf{A}_{2}+16 \mathbf{A}_{2}^{2} \mathbf{A}_{4} \mathbf{A}_{2}+24 \mathbf{A}_{2}^{3} \mathbf{A}_{4}$

$\left.+216 \mathbf{A}_{2}^{6}-104 \mathbf{A}_{2}^{4} \mathbf{A}_{3}\right) \mathbf{e}_{0}^{6}+\left(36 \mathbf{A}_{2}^{2} \mathbf{A}_{3} \mathbf{A}_{4}\right.$ 


$$
\begin{aligned}
& +36 \mathbf{A}_{2} \mathbf{A}_{3} \mathbf{A}_{2} \mathbf{A}_{4}+296 \mathbf{A}_{2}^{2} \mathbf{A}_{3} \mathbf{A}_{2}^{3}+300 \mathbf{A}_{2}^{3} \mathbf{A}_{3} \mathbf{A}_{2}^{2} \\
& +20 \mathbf{A}_{2} \mathbf{A}_{5} \mathbf{A}_{2}^{2}+24 \mathbf{A}_{2} \mathbf{A}_{4} \mathbf{A}_{3} \mathbf{A}_{2}+24 \mathbf{A}_{2} \mathbf{A}_{3} \mathbf{A}_{4} \mathbf{A}_{2} \\
& +20 \mathbf{A}_{2}^{2} \mathbf{A}_{5} \mathbf{A}_{2}+336 \mathbf{A}_{2}^{4} \mathbf{A}_{3} \mathbf{A}_{2}-156 \mathbf{A}_{2} \mathbf{A}_{3} \mathbf{A}_{2}^{2} \mathbf{A}_{3} \\
& -144 \mathbf{A}_{2}^{2} \mathbf{A}_{3} \mathbf{A}_{2} \mathbf{A}_{3}-160 \mathbf{A}_{2}^{3} \mathbf{A}_{3}^{2}-156 \mathbf{A}_{2}^{4} \mathbf{A}_{4} \\
& +336 \mathbf{A}_{2} \mathbf{A}_{3} \mathbf{A}_{2}^{4}+36 \mathbf{A}_{2} \mathbf{A}_{3}^{3}+32 \mathbf{A}_{2} \mathbf{A}_{4} \mathbf{A}_{2} \mathbf{A}_{3} \\
& -108 \mathbf{A}_{2}^{3} \mathbf{A}_{4} \mathbf{A}_{2}+32 \mathbf{A}_{2}^{2} \mathbf{A}_{4} \mathbf{A}_{3}-108 \mathbf{A}_{2}^{2} \mathbf{A}_{3}^{2} \mathbf{A}_{2} \\
& -120 \mathbf{A}_{2} \mathbf{A}_{3} \mathbf{A}_{2} \mathbf{A}_{3} \mathbf{A}_{2}-104 \mathbf{A}_{2} \mathbf{A}_{4} \mathbf{A}_{2}^{3}-108 \mathbf{A}_{2} \mathbf{A}_{3}^{2} \mathbf{A}_{2}^{2} \\
& \left.-96 \mathbf{A}_{2}^{2} \mathbf{A}_{4} \mathbf{A}_{2}^{2}+32 \mathbf{A}_{2}^{3} \mathbf{A}_{5}+432 \mathbf{A}_{2}^{5} \mathbf{A}_{3}-712 \mathbf{A}_{2}^{7}\right) \mathbf{e}_{0}^{7} \\
& \left.+\cdots+O\left(\mathbf{e}_{0}^{10}\right)\right) \text {, } \\
& \phi_{4}=4 \mathbf{A}_{2}^{3} \mathbf{e}_{0}^{4}+\left(-34 \mathbf{A}_{2}^{4}+6 \mathbf{A}_{2} \mathbf{A}_{3} \mathbf{A}_{2}+6 \mathbf{A}_{3} \mathbf{A}_{2}^{2}\right. \\
& \left.+8 \mathbf{A}_{2}^{2} \mathbf{A}_{3}\right) \mathbf{e}_{0}^{5}+\left(12 \mathbf{A}_{2} \mathbf{A}_{3}^{2}-68 \mathbf{A}_{2}^{3} \mathbf{A}_{3}+176 \mathbf{A}_{2}^{5}\right. \\
& +12 \mathbf{A}_{2}^{2} \mathbf{A}_{4}+8 \mathbf{A}_{2} \mathbf{A}_{4} \mathbf{A}_{2}-52 \mathbf{A}_{2}^{2} \mathbf{A}_{3} \mathbf{A}_{2}-48 \mathbf{A}_{2} \mathbf{A}_{3} \mathbf{A}_{2}^{2} \\
& \left.-51 \mathbf{A}_{3} \mathbf{A}_{2}^{3}+8 \mathbf{A}_{4} \mathbf{A}_{2}^{2}+9 \mathbf{A}_{3}^{2} \mathbf{A}_{2}+12 \mathbf{A}_{3} \mathbf{A}_{2} \mathbf{A}_{3}\right) \mathbf{e}_{0}^{6} \\
& +\left(-104 \mathbf{A}_{2}^{2} \mathbf{A}_{3}^{2}-96 \mathbf{A}_{2} \mathbf{A}_{3} \mathbf{A}_{2} \mathbf{A}_{3}-102 \mathbf{A}_{3} \mathbf{A}_{2}^{2} \mathbf{A}_{3}\right. \\
& +16 \mathbf{A}_{4} \mathbf{A}_{2} \mathbf{A}_{3}+18 \mathbf{A}_{3}^{3}+16 \mathbf{A}_{2} \mathbf{A}_{4} \mathbf{A}_{3}+18 \mathbf{A}_{2} \mathbf{A}_{3} \mathbf{A}_{4} \\
& +18 \mathbf{A}_{3} \mathbf{A}_{2} \mathbf{A}_{4}+352 \mathbf{A}_{2}^{4} \mathbf{A}_{3}-102 \mathbf{A}_{2}^{3} \mathbf{A}_{4}-708 \mathbf{A}_{2}^{6} \\
& +10 \mathbf{A}_{5} \mathbf{A}_{2}^{2}+12 \mathbf{A}_{4} \mathbf{A}_{3} \mathbf{A}_{2}+12 \mathbf{A}_{3} \mathbf{A}_{4} \mathbf{A}_{2}+10 \mathbf{A}_{2} \mathbf{A}_{5} \mathbf{A}_{2} \\
& +272 \mathbf{A}_{2}^{3} \mathbf{A}_{3} \mathbf{A}_{2}+246 \mathbf{A}_{2}^{2} \mathbf{A}_{3} \mathbf{A}_{2}^{2}+250 \mathbf{A}_{2} \mathbf{A}_{3} \mathbf{A}_{2}^{3} \\
& +270 A_{3} A_{2}^{4}+16 A_{2}^{2} A_{5}-68 A_{4} A_{2}^{3}-72 A_{3}^{2} A_{2}^{2} \\
& -64 \mathbf{A}_{2} \mathbf{A}_{4} \mathbf{A}_{2}^{2}-72 \mathbf{A}_{2} \mathbf{A}_{3}^{2} \mathbf{A}_{2}-78 \mathbf{A}_{3} \mathbf{A}_{2} \mathbf{A}_{3} \mathbf{A}_{2} \\
& \left.-70 \mathbf{A}_{2}^{2} \mathbf{A}_{4} \mathbf{A}_{2}\right) \mathbf{e}_{0}^{7}+\cdots+O\left(\mathbf{e}_{0}^{10}\right), \\
& \phi_{5}=4 \mathbf{A}_{2}^{3} \mathbf{e}_{0}^{4}+\left(-42 \mathbf{A}_{2}^{4}+6 \mathbf{A}_{2} \mathbf{A}_{3} \mathbf{A}_{2}+6 \mathbf{A}_{3} \mathbf{A}_{2}^{2}\right. \\
& \left.+8 \mathbf{A}_{2}^{2} \mathbf{A}_{3}\right) \mathbf{e}_{0}^{5}+\left(8 \mathbf{A}_{2} \mathbf{A}_{4} \mathbf{A}_{2}+12 \mathbf{A}_{3} \mathbf{A}_{2} \mathbf{A}_{3}\right. \\
& -64 A_{2}^{2} A_{3} A_{2}-60 A_{2} A_{3} A_{2}^{2}-63 A_{3} A_{2}^{3}+8 A_{4} A_{2}^{2} \\
& +9 \mathbf{A}_{3}^{2} \mathbf{A}_{2}+12 \mathbf{A}_{2} \mathbf{A}_{3}^{2}-84 \mathbf{A}_{2}^{3} \mathbf{A}_{3}+260 \mathbf{A}_{2}^{5} \\
& \left.+12 \mathbf{A}_{2}^{2} \mathbf{A}_{4}\right) \mathbf{e}_{0}^{6}+\left(-128 \mathbf{A}_{2}^{2} \mathbf{A}_{3}^{2}-120 \mathbf{A}_{2} \mathbf{A}_{3} \mathbf{A}_{2} \mathbf{A}_{3}\right. \\
& -126 \mathbf{A}_{3} \mathbf{A}_{2}^{2} \mathbf{A}_{3}+16 \mathbf{A}_{4} \mathbf{A}_{2} \mathbf{A}_{3}+18 \mathbf{A}_{3}^{3}+16 \mathbf{A}_{2} \mathbf{A}_{4} \mathbf{A}_{3} \\
& +18 \mathbf{A}_{2} \mathbf{A}_{3} \mathbf{A}_{4}+18 \mathbf{A}_{3} \mathbf{A}_{2} \mathbf{A}_{4}+520 \mathbf{A}_{2}^{4} \mathbf{A}_{3}-126 \mathbf{A}_{2}^{3} \mathbf{A}_{4} \\
& -1228 \mathbf{A}_{2}^{6}+10 \mathbf{A}_{5} \mathbf{A}_{2}^{2}+12 \mathbf{A}_{4} \mathbf{A}_{3} \mathbf{A}_{2}+12 \mathbf{A}_{3} \mathbf{A}_{4} \mathbf{A}_{2} \\
& +10 \mathbf{A}_{2} \mathbf{A}_{5} \mathbf{A}_{2}+400 \mathbf{A}_{2}^{3} \mathbf{A}_{3} \mathbf{A}_{2}+366 \mathbf{A}_{2}^{2} \mathbf{A}_{3} \mathbf{A}_{2}^{2} \\
& +376 \mathbf{A}_{2} \mathbf{A}_{3} \mathbf{A}_{2}^{3}+396 \mathbf{A}_{3} \mathbf{A}_{2}^{4}+16 \mathbf{A}_{2}^{2} \mathbf{A}_{5}-84 \mathbf{A}_{4} \mathbf{A}_{2}^{3} \\
& -90 \mathbf{A}_{3}^{2} \mathbf{A}_{2}^{2}-80 \mathbf{A}_{2} \mathbf{A}_{4} \mathbf{A}_{2}^{2}-90 \mathbf{A}_{2} \mathbf{A}_{3}^{2} \mathbf{A}_{2}
\end{aligned}
$$

$$
\begin{aligned}
& \left.-96 \mathbf{A}_{3} \mathbf{A}_{2} \mathbf{A}_{3} \mathbf{A}_{2}-86 \mathbf{A}_{2}^{2} \mathbf{A}_{4} \mathbf{A}_{2}\right) \mathbf{e}_{0}^{7}+\cdots+O\left(\mathbf{e}_{0}^{10}\right) \\
& \phi_{6}=4 \mathbf{A}_{2}^{3} \mathbf{e}_{0}^{4}+\left(-50 \mathbf{A}_{2}^{4}+6 \mathbf{A}_{2} \mathbf{A}_{3} \mathbf{A}_{2}+6 \mathbf{A}_{3} \mathbf{A}_{2}^{2}\right. \\
& \left.+8 \mathbf{A}_{2}^{2} \mathbf{A}_{3}\right) \mathbf{e}_{0}^{5}+\left(-76 \mathbf{A}_{2}^{2} \mathbf{A}_{3} \mathbf{A}_{2}-72 \mathbf{A}_{2} \mathbf{A}_{3} \mathbf{A}_{2}^{2}\right. \\
& -75 \mathbf{A}_{3} \mathbf{A}_{2}^{3}+8 \mathbf{A}_{4} \mathbf{A}_{2}^{2}+9 \mathbf{A}_{3}^{2} \mathbf{A}_{2}+8 \mathbf{A}_{2} \mathbf{A}_{4} \mathbf{A}_{2} \\
& +12 \mathbf{A}_{3} \mathbf{A}_{2} \mathbf{A}_{3}+12 \mathbf{A}_{2} \mathbf{A}_{3}^{2}-100 \mathbf{A}_{2}^{3} \mathbf{A}_{3}+360 \mathbf{A}_{2}^{5} \\
& \left.+12 \mathbf{A}_{2}^{2} \mathbf{A}_{4}\right) \mathbf{e}_{0}^{6}+\left(-152 \mathbf{A}_{2}^{2} \mathbf{A}_{3}^{2}-144 \mathbf{A}_{2} \mathbf{A}_{3} \mathbf{A}_{2} \mathbf{A}_{3}\right. \\
& -150 \mathbf{A}_{3} \mathbf{A}_{2}^{2} \mathbf{A}_{3}+16 \mathbf{A}_{4} \mathbf{A}_{2} \mathbf{A}_{3}+18 \mathbf{A}_{3}^{3}+16 \mathbf{A}_{2} \mathbf{A}_{4} \mathbf{A}_{3} \\
& +18 \mathbf{A}_{2} \mathbf{A}_{3} \mathbf{A}_{4}+18 \mathbf{A}_{3} \mathbf{A}_{2} \mathbf{A}_{4}+720 \mathbf{A}_{2}^{4} \mathbf{A}_{3}-150 \mathbf{A}_{2}^{3} \mathbf{A}_{4} \\
& -1948 \mathbf{A}_{2}^{6}+10 \mathbf{A}_{5} \mathbf{A}_{2}^{2}+12 \mathbf{A}_{4} \mathbf{A}_{3} \mathbf{A}_{2}+12 \mathbf{A}_{3} \mathbf{A}_{4} \mathbf{A}_{2} \\
& +10 \mathbf{A}_{2} \mathbf{A}_{5} \mathbf{A}_{2}+552 \mathbf{A}_{2}^{3} \mathbf{A}_{3} \mathbf{A}_{2}+510 \mathbf{A}_{2}^{2} \mathbf{A}_{3} \mathbf{A}_{2}^{2} \\
& +526 \mathbf{A}_{2} \mathbf{A}_{3} \mathbf{A}_{2}^{3}+546 \mathbf{A}_{3} \mathbf{A}_{2}^{4}+16 \mathbf{A}_{2}^{2} \mathbf{A}_{5}-100 \mathbf{A}_{4} \mathbf{A}_{2}^{3} \\
& -108 \mathbf{A}_{3}^{2} \mathbf{A}_{2}^{2}-96 \mathbf{A}_{2} \mathbf{A}_{4} \mathbf{A}_{2}^{2}-108 \mathbf{A}_{2} \mathbf{A}_{3}^{2} \mathbf{A}_{2} \\
& \left.-114 \mathbf{A}_{3} \mathbf{A}_{2} \mathbf{A}_{3} \mathbf{A}_{2}-102 \mathbf{A}_{2}^{2} \mathbf{A}_{4} \mathbf{A}_{2}\right) \mathbf{e}_{0}^{7}+\cdots+O\left(\mathbf{e}_{0}^{10}\right), \\
& \phi_{7}=4 \mathbf{A}_{2}^{3} \mathbf{e}_{0}^{4}+\left(-58 \mathbf{A}_{2}^{4}+6 \mathbf{A}_{2} \mathbf{A}_{3} \mathbf{A}_{2}+6 \mathbf{A}_{3} \mathbf{A}_{2}^{2}\right. \\
& \left.+8 \mathbf{A}_{2}^{2} \mathbf{A}_{3}\right) \mathbf{e}_{0}^{5}+\left(12 \mathbf{A}_{3} \mathbf{A}_{2} \mathbf{A}_{3}+8 \mathbf{A}_{2} \mathbf{A}_{4} \mathbf{A}_{2}+476 \mathbf{A}_{2}^{5}\right. \\
& +12 \mathbf{A}_{2}^{2} \mathbf{A}_{4}+12 \mathbf{A}_{2} \mathbf{A}_{3}^{2}-116 \mathbf{A}_{2}^{3} \mathbf{A}_{3}-88 \mathbf{A}_{2}^{2} \mathbf{A}_{3} \mathbf{A}_{2} \\
& \left.-84 \mathbf{A}_{2} \mathbf{A}_{3} \mathbf{A}_{2}^{2}-87 \mathbf{A}_{3} \mathbf{A}_{2}^{3}+8 \mathbf{A}_{4} \mathbf{A}_{2}^{2}+9 \mathbf{A}_{3}^{2} \mathbf{A}_{2}\right) \mathbf{e}_{0}^{6} \\
& +\left(-176 \mathbf{A}_{2}^{2} \mathbf{A}_{3}^{2}-168 \mathbf{A}_{2} \mathbf{A}_{3} \mathbf{A}_{2} \mathbf{A}_{3}-174 \mathbf{A}_{3} \mathbf{A}_{2}^{2} \mathbf{A}_{3}\right. \\
& +16 \mathbf{A}_{4} \mathbf{A}_{2} \mathbf{A}_{3}+18 \mathbf{A}_{3}^{3}+16 \mathbf{A}_{2} \mathbf{A}_{4} \mathbf{A}_{3}+18 \mathbf{A}_{2} \mathbf{A}_{3} \mathbf{A}_{4} \\
& +18 \mathbf{A}_{3} \mathbf{A}_{2} \mathbf{A}_{4}+952 \mathbf{A}_{2}^{4} \mathbf{A}_{3}-174 \mathbf{A}_{2}^{3} \mathbf{A}_{4}-2900 \mathbf{A}_{2}^{6} \\
& +10 \mathbf{A}_{5} \mathbf{A}_{2}^{2}+12 \mathbf{A}_{4} \mathbf{A}_{3} \mathbf{A}_{2}+12 \mathbf{A}_{3} \mathbf{A}_{4} \mathbf{A}_{2}+10 \mathbf{A}_{2} \mathbf{A}_{5} \mathbf{A}_{2} \\
& +728 \mathbf{A}_{2}^{3} \mathbf{A}_{3} \mathbf{A}_{2}+678 \mathbf{A}_{2}^{2} \mathbf{A}_{3} \mathbf{A}_{2}^{2}+700 \mathbf{A}_{2} \mathbf{A}_{3} \mathbf{A}_{2}^{3} \\
& +720 \mathbf{A}_{3} \mathbf{A}_{2}^{4}+16 \mathbf{A}_{2}^{2} \mathbf{A}_{5}-116 \mathbf{A}_{4} \mathbf{A}_{2}^{3}-126 \mathbf{A}_{3}^{2} \mathbf{A}_{2}^{2} \\
& -112 \mathbf{A}_{2} \mathbf{A}_{4} \mathbf{A}_{2}^{2}-126 \mathbf{A}_{2} \mathbf{A}_{3}^{2} \mathbf{A}_{2}-132 \mathbf{A}_{3} \mathbf{A}_{2} \mathbf{A}_{3} \mathbf{A}_{2} \\
& \left.-118 \mathbf{A}_{2}^{2} \mathbf{A}_{4} \mathbf{A}_{2}\right) \mathbf{e}_{0}^{7}+\cdots+O\left(\mathbf{e}_{0}^{10}\right), \\
& \phi_{8}=4 \mathbf{A}_{2}^{3} \mathbf{e}_{0}^{4}+\left(-66 \mathbf{A}_{2}^{4}+6 \mathbf{A}_{2} \mathbf{A}_{3} \mathbf{A}_{2}+6 \mathbf{A}_{3} \mathbf{A}_{2}^{2}\right. \\
& \left.+8 \mathbf{A}_{2}^{2} \mathbf{A}_{3}\right) \mathbf{e}_{0}^{5}+\left(12 \mathbf{A}_{3} \mathbf{A}_{2} \mathbf{A}_{3}+12 \mathbf{A}_{2} \mathbf{A}_{3}^{2}-132 \mathbf{A}_{2}^{3} \mathbf{A}_{3}\right. \\
& +608 \mathbf{A}_{2}^{5}+12 \mathbf{A}_{2}^{2} \mathbf{A}_{4}-100 \mathbf{A}_{2}^{2} \mathbf{A}_{3} \mathbf{A}_{2}-96 \mathbf{A}_{2} \mathbf{A}_{3} \mathbf{A}_{2}^{2} \\
& \left.-99 \mathbf{A}_{3} \mathbf{A}_{2}^{3}+8 \mathbf{A}_{4} \mathbf{A}_{2}^{2}+9 \mathbf{A}_{3}^{2} \mathbf{A}_{2}+8 \mathbf{A}_{2} \mathbf{A}_{4} \mathbf{A}_{2}\right) \mathbf{e}_{0}^{6} \\
& +\left(-200 \mathbf{A}_{2}^{2} \mathbf{A}_{3}^{2}-192 \mathbf{A}_{2} \mathbf{A}_{3} \mathbf{A}_{2} \mathbf{A}_{3}-198 \mathbf{A}_{3} \mathbf{A}_{2}^{2} \mathbf{A}_{3}\right. \\
& +16 \mathbf{A}_{4} \mathbf{A}_{2} \mathbf{A}_{3}+18 \mathbf{A}_{3}^{3}+16 \mathbf{A}_{2} \mathbf{A}_{4} \mathbf{A}_{3}+18 \mathbf{A}_{2} \mathbf{A}_{3} \mathbf{A}_{4} \\
& +18 \mathbf{A}_{3} \mathbf{A}_{2} \mathbf{A}_{4}+1216 \mathbf{A}_{2}^{4} \mathbf{A}_{3}-198 \mathbf{A}_{2}^{3} \mathbf{A}_{4}-4116 \mathbf{A}_{2}^{6}
\end{aligned}
$$




$$
\begin{aligned}
& +10 \mathbf{A}_{5} \mathbf{A}_{2}^{2}+12 \mathbf{A}_{4} \mathbf{A}_{3} \mathbf{A}_{2}+12 \mathbf{A}_{3} \mathbf{A}_{4} \mathbf{A}_{2}+10 \mathbf{A}_{2} \mathbf{A}_{5} \mathbf{A}_{2} \\
& +928 \mathbf{A}_{2}^{3} \mathbf{A}_{3} \mathbf{A}_{2}+870 \mathbf{A}_{2}^{2} \mathbf{A}_{3} \mathbf{A}_{2}^{2}+898 \mathbf{A}_{2} \mathbf{A}_{3} \mathbf{A}_{2}^{3} \\
& +918 \mathbf{A}_{3} \mathbf{A}_{2}^{4}+16 \mathbf{A}_{2}^{2} \mathbf{A}_{5}-132 \mathbf{A}_{4} \mathbf{A}_{2}^{3}-144 \mathbf{A}_{3}^{2} \mathbf{A}_{2}^{2} \\
& -128 \mathbf{A}_{2} \mathbf{A}_{4} \mathbf{A}_{2}^{2}-144 \mathbf{A}_{2} \mathbf{A}_{3}^{2} \mathbf{A}_{2}-150 \mathbf{A}_{3} \mathbf{A}_{2} \mathbf{A}_{3} \mathbf{A}_{2} \\
& \left.-134 \mathbf{A}_{2}^{2} \mathbf{A}_{4} \mathbf{A}_{2}\right) \mathbf{e}_{0}^{7}+\cdots+O\left(\mathbf{e}_{0}^{10}\right) \\
\mathbf{e}_{4} & =\left(184 \mathbf{A}_{2}^{8}+8 \mathbf{A}_{2}^{3} \mathbf{A}_{3} \mathbf{A}_{2}^{3}-24 \mathbf{A}_{3} \mathbf{A}_{2}^{6}\right) \mathbf{e}_{0}^{9}+O\left(\mathbf{e}_{0}^{10}\right)
\end{aligned}
$$

Now, we present the proof of convergence of NMIM via induction.

Theorem 2. The convergence order of NMIM for $m \geq 4$ is $5 m-11$.

Proof. All the computations are made under the assumption of Theorem 1 . We know from Theorem 1 that the convergence order of NMIM method is nine for $m=4$. By performing the computations in a similar manner to that of Theorem 1, we get the following error equation for $m=5$ :

$$
\begin{aligned}
\mathbf{e}_{5}= & \left(4 \mathbf{A}_{2}^{3} \mathbf{A}_{3}-12 \mathbf{A}_{3} \mathbf{A}_{2}^{3}+60 \mathbf{A}_{2}^{5}\right) \\
& \cdot\left(184 \mathbf{A}_{2}^{8}+8 \mathbf{A}_{2}^{3} \mathbf{A}_{3} \mathbf{A}_{2}^{3}-24 \mathbf{A}_{3} \mathbf{A}_{2}^{6}\right) \mathbf{e}_{0}^{14}+O\left(\mathbf{e}_{0}^{15}\right) .
\end{aligned}
$$

This proves that the convergence order of NMIM is $5 m-11$ for $m=5$. Now, assume that the convergence order of NMIM is $5 m-11$ for a number of steps' $m \geq 5$ and and let us prove that the convergence order of NMIM for $m$ steps is $5 m-6$ for $(m)$ th step. If the convergence order of NMIM for $m$ steps is $5 m-11$, then

$$
\mathbf{e}_{m}=\mathbf{y}_{m}-\mathbf{y}^{*} \sim \mathbf{d e}_{0}^{5 m-11},
$$

where $\mathbf{d}$ is the asymptotic constant and symbol $\sim$ means "approximately equal to." By using (17), we perform the following steps:

$$
\begin{aligned}
& \mathbf{F}\left(\mathbf{y}_{0}\right)^{-1} \sim\left(\mathbf{I}-2 \mathbf{A}_{2} \mathbf{e}_{0}\right) \mathbf{A}_{1}^{-1}, \\
& \mathbf{F}^{\prime}\left(\mathbf{y}_{3}\right) \sim \mathbf{A}_{1}\left(\mathbf{I}+8 \mathbf{A}_{2}^{4} \mathbf{e}_{0}^{4}\right), \\
& \mathbf{F}\left(\mathbf{y}_{s}\right) \sim \mathbf{A}_{1} \mathbf{d e}_{0}^{5 m-11}, \\
& \phi_{9} \sim\left(\mathbf{d}-2 \mathbf{A}_{2} \mathbf{d e} \mathbf{e}_{0}\right) \mathbf{e}_{0}^{5 m-11}, \\
& \phi_{10} \sim\left(\mathbf{d}-4 \mathbf{A}_{2} \mathbf{d e}_{0}+4 \mathbf{A}_{2}^{2} \mathbf{d e}_{0}^{2}+8 \mathbf{A}_{2}^{4} \mathbf{d e}_{0}^{4}-32 \mathbf{A}_{2}^{5} \mathbf{d e}_{0}^{5}\right) \\
& \cdot \mathbf{e}_{0}^{5 m-11} \\
& \phi_{11} \sim\left(\mathbf{d}-6 \mathbf{A}_{2} \mathbf{d e}_{0}+12 \mathbf{A}_{2}^{2} \mathbf{d e}_{0}^{2}-8 \mathbf{A}_{2}^{3} \mathbf{d e}_{0}^{3}+16 \mathbf{A}_{2}^{4} \mathbf{d e} \mathbf{e}_{0}^{4}\right. \\
& \left.-96 \mathbf{A}_{2}^{5} \mathbf{d e} \mathbf{e}_{0}^{5}\right) \mathbf{e}_{0}^{5 m-11}
\end{aligned}
$$

$$
\begin{aligned}
& \phi_{12} \sim\left(\mathbf{d}-8 \mathbf{A}_{2} \mathbf{d e}_{0}+24 \mathbf{A}_{2}^{2} \mathbf{d e}_{0}^{2}-32 \mathrm{~A}_{2}^{3} \mathbf{d e}_{0}^{3}+40 \mathbf{A}_{2}^{4} \mathbf{d e}_{0}^{4}\right. \\
& \left.-192 \mathbf{A}_{2}^{5} \mathbf{d e}_{0}^{5}\right) \mathbf{e}_{0}^{5 m-11} \\
& \phi_{13} \sim\left(\mathbf{d}-10 \mathbf{A}_{2} \mathbf{d e}_{0}+40 \mathbf{A}_{2}^{2} \mathbf{d e}_{0}^{2}-80 \mathbf{A}_{2}^{3} \mathbf{d e}_{0}^{3}\right. \\
& \left.+112 \mathbf{A}_{2}^{4} \mathbf{d e} \mathbf{e}_{0}^{4}-352 \mathbf{A}_{2}^{5} \mathbf{d e} \mathbf{e}_{0}^{5}\right) \mathbf{e}_{0}^{5 m-11}, \\
& \mathbf{e}_{m+1} \sim 48 \mathbf{A}_{2}^{5} \mathbf{d e}_{0}^{5 m-6} \text {. }
\end{aligned}
$$

This completes the proof that the convergence order of NMIM is $5 m-6$ for $m+1$ steps.

\section{Computational Cost}

The computational costs in terms of multiplications of different iterative methods are shown in Table 1 , where $m$ is the number of steps, $n$ is the number of dimensions of the problem, $l$ is the ratio between the computational cost of a division and the computational cost of a multiplication, $\alpha$ is the ratio between the computational cost of a function component evaluation and the computational cost of a multiplication, $\beta$ is the ratio between the computational cost of a component of the Jacobian and the computational cost of a multiplication, and $\gamma$ is the ratio between the computational cost of a second derivative and the computational cost of a multiplication. Table 2 displays the difference of computational costs of methods with respect to NMIM when the methods have the same convergence order as NMIM with $m$ steps. Table 3 gives the conditions on $\alpha$ and bounds on $m$ so that, for the same convergence order, the computational cost of NMIM is smaller than that of the other methods.

\section{Numerical Simulations}

In this section, we verify the convergence order of NMIM and solve fifteen nonlinear initial and boundary value problems to show the validity, accuracy, and efficiency of our proposed iterative method. For the discretization, we use four pseudospectral collocation methods. In all experiments but one, we apply NMIM once with several steps to reduce the absolute error in the computed numerical solution. To verify the convergence order computationally, we adopt the following definition of computational convergence order (CCO):

$$
\mathrm{CCO}=\frac{\log \left(\left\|\mathbf{F}\left(\mathbf{x}_{k+1}\right)\right\|_{\infty} /\left\|\mathbf{F}\left(\mathbf{x}_{k}\right)\right\|_{\infty}\right)}{\log \left(\left\|\mathbf{F}\left(\mathbf{x}_{k}\right)\right\|_{\infty} /\left\|\mathbf{F}\left(\mathbf{x}_{k-1}\right)\right\|_{\infty}\right)} .
$$

5.1. Computational Verification of Convergence Order. In general, it is hard to verify the convergence order by solving initial and boundary value problems. Therefore, we consider the following five systems of nonlinear equations $\mathbf{F}(\mathbf{x})=\mathbf{0}$. 


$$
\begin{aligned}
& \text { Problem } 1=\left\{\begin{array}{l}
F_{1}(\mathbf{x})=x_{2} x_{3}+x_{4}\left(x_{2}+x_{3}\right), \\
F_{2}(\mathbf{x})=x_{1} x_{3}+x_{4}\left(x_{1}+x_{3}\right), \\
F_{3}(\mathbf{x})=x_{1} x_{2}+x_{4}\left(x_{1}+x_{2}\right), \\
F_{4}(\mathbf{x})=x_{1} x_{2}+x_{3}\left(x_{1}+x_{2}\right)-1,
\end{array}\right. \\
& \text { Problem } 2= \begin{cases}F_{i}(\mathbf{x})=x_{i}^{2} x_{i+1}-1, & i=1,2, \ldots, n-1, \\
F_{i}(\mathbf{x})=x_{n} x_{1}-1, & i=n,\end{cases} \\
& \text { Problem } 3=\left\{\begin{array}{l}
F_{1}(\mathbf{x})=\left(3-0.5 x_{1}\right) x_{1}-2 x_{2}+1, \\
F_{2}(\mathbf{x})=\left(3-0.5 x_{n}\right) x_{n}-2 x_{n-1}+1, \\
F_{i}(\mathbf{x})=\left(3-0.5 x_{i}\right) x_{i}-x_{i-1}+2 x_{i+1}+1, \quad i=2,3, \ldots, n-1,
\end{array}\right. \\
& \text { Problem } 4=\left\{F_{i}(\mathbf{x})=2\left(n+i\left(1-\cos \left(x_{i}\right)\right)-\sin \left(x_{i}\right)-\sum_{j=1}^{n} \cos \left(x_{j}\right)\right)\left(2 \sin \left(x_{i}\right)-\cos \left(x_{i}\right)\right), i=1,2, \ldots, n,\right. \\
& \text { Problem } 5=\left\{\begin{array}{l}
F_{1}(\mathbf{x})=10 x_{1}+\sin \left(x_{1}+x_{2}\right)-1, \\
F_{2}(\mathbf{x})=8 x_{2}-\cos ^{2}\left(x_{3}-x_{2}\right)-1, \\
F_{3}(\mathbf{x})=12 x_{3}+\sin \left(x_{3}\right)-1 .
\end{array}\right.
\end{aligned}
$$

The system of nonlinear equations is solved by Mathematica using 11,000 digits. Table 4 shows that CCOs agree with theoretical convergence orders.

5.2. Solving Initial and Boundary Value Problems. In this section, we solve different initial and boundary value problems by using different J-GL-C discretization methods. In all computations, we make spatial and temporal discretizations to translate the entire IVPs and BVPs into systems of nonlinear equations. The systems of nonlinear equations are then solved by using the proposed iterative method NMIM. In most of the problems, we use vector $\mathbf{0}$ with initial and boundary conditions introduced in it as the initial guess. The inclusion of initial and boundary conditions in $\mathbf{0}$ makes the initial guess nonsmooth but it works for most of the problems. When the nonsmooth initial guess does not work, we then make it smooth to get convergence.

5.2.1. The Lane-Emden Equation. The Lane-Emden equation is

$$
\begin{aligned}
x^{\prime \prime}(t)+\frac{2}{t} x^{\prime}(t)+x(t)^{p} & =0, \\
x^{\prime}(0) & =0, \\
x(0) & =1 .
\end{aligned}
$$

J-GL-C discretization methods translate the Lane-Emden equation into the following system of nonlinear equations:

$$
\begin{aligned}
& \mathbf{F}(\mathbf{x})=\mathbf{S}_{x x} \mathbf{x}+\operatorname{diag}\left(\frac{2}{\mathbf{t}}\right) \mathbf{S}_{x} \mathbf{x}+\mathbf{x}^{p}=\mathbf{0}, \\
& \mathbf{F}^{\prime}(\mathbf{x})=\mathbf{S}_{x x}+\operatorname{diag}\left(\frac{2}{\mathbf{t}}\right) \mathbf{S}_{x}+p \operatorname{diag}\left(\mathbf{x}^{p-1}\right),
\end{aligned}
$$

where $\operatorname{diag}(\cdot)$ denotes a diagonal matrix with values in the diagonal equal to the elements of its vector argument, $\mathbf{S}_{x}=$ $2 / b \mathbf{Q}_{x}, \mathbf{S}_{x x}=\left(2 / b \mathbf{Q}_{x}\right)^{2}$, and $[0, b]$ is the domain of the problem. We have solved the Lane-Emden equation for different values of $p$ ranging from two to five and $b=3$. Figure 1 shows the initial guess and the computed numerical solutions for $\theta=1 / 2, \phi=-1 / 2$, and 50 grid points. Table 5 gives the norm of the residue in the solution of the nonlinear systems by a single iteration of NMIM with varying $m$.

5.2.2. Bratu Problem. Bratu problem is the boundary value problem:

$$
\begin{aligned}
x^{\prime \prime}(t)+\alpha e^{x(t)} & =0, \\
x(0) & =0, \\
x(1) & =0,
\end{aligned}
$$


TABLE 1: Computational costs of different iterative methods.

\begin{tabular}{lc}
\hline Methods & Computational cost \\
\hline MNR & $\frac{1}{3} n^{3}+\left(\beta+m-\frac{1}{2}+\frac{l}{2}\right) n^{2}+\left(m \alpha+l m-m+\frac{1}{6}-\frac{l}{2}\right) n$ \\
HJ & $\frac{1}{3} n^{3}+\left(2 \beta-\frac{3}{2}+\frac{l}{2}+3 m\right) n^{2}+\left(m \alpha-\alpha+\frac{7}{6}-\frac{3}{2} l+2 l m\right) n$ \\
FTUC & $\frac{1}{3} n^{3}+\left(2 \beta-\frac{7}{2}+\frac{l}{2}+3 m\right) n^{2}+\left(m \alpha-\alpha+\frac{19}{6}-\frac{5}{2} l-m+2 l m\right) n$ \\
MSF & $\frac{1}{3} n^{3}+\left(2 \beta-\frac{7}{2}+\frac{l}{2}+5 m\right) n^{2}+\left(m \alpha+\gamma+\frac{19}{6}-\frac{3}{2} l-2 m+3 l m\right) n$ \\
NMIM & $\frac{1}{3} n^{3}+\left(2 \beta-\frac{49}{2}+\frac{l}{2}+9 m\right) n^{2}+\left(\alpha m-\frac{17}{6}-\frac{25}{2} l+5 l m\right) n$ \\
\hline
\end{tabular}

TABLE 2: Difference of computational costs of different iterative methods with respect to NMIM when the methods have the same convergence order as of NMIM with $m$ steps.

\begin{tabular}{lr}
\hline Methods & Difference of computational costs \\
\hline MNR-NMIM & $4 n \alpha m-\beta n^{2}-4 n^{2} m-10 n \alpha+2 l n-5 n m+14 n^{2}+13 n$ \\
HJ-NMIM & $\frac{13}{2} n^{2}-\frac{3}{2} n^{2} m+\frac{3}{2} n \alpha m-\frac{13}{2} n \alpha+4 n$ \\
FTUC-NMIM & $14 n^{2}-4 n^{2} m+\frac{2}{3} n \alpha m-\frac{10}{3} n \alpha+\frac{25 n}{3}+\frac{16}{3} l n-\frac{5}{3} n m-\frac{5}{3} l m n$ \\
MSF-NMIM & $-\frac{2}{3} n^{2} m+\frac{8}{3} n^{2}+\frac{2}{3} n \alpha m-\frac{11}{3} n \alpha+\gamma n+\frac{40 n}{3}-\frac{10}{3} n m$ \\
\hline
\end{tabular}

TABLE 3: Conditions and bounds on $m$ so that, for the same convergence order, the computational cost of NMIM is smaller.

\begin{tabular}{lcr}
\hline Computational cost & Bounds on $m$ & Conditions \\
\hline$C_{\mathrm{MNR}}>C_{\mathrm{NMIM}}$ & $m<\frac{n \beta+10 \alpha-2 l-14 n-13}{-4 n+4 \alpha-5}$ & $\alpha<n+\frac{5}{4}$ \\
$C_{\mathrm{HJ}}>C_{\mathrm{NMIM}}$ & $m<\frac{1}{3} \frac{-13 n+13 \alpha-8}{-n+\alpha}$ & $\alpha<n$ \\
$C_{\mathrm{FTUC}}>C_{\mathrm{NMIM}}$ & $m<\frac{-42 n-16 l+10 \alpha-25}{-12 n-5 l+2 \alpha-5}$ & $\alpha<6 n+\frac{5}{2}+\frac{5}{2} l$ \\
$C_{\mathrm{MSF}}>C_{\mathrm{NMIM}}$ & $m<-\frac{1}{2} \frac{8 n-11 \alpha+3 \gamma+40}{-n+\alpha-5}$ & $\alpha<n+5$ \\
\hline
\end{tabular}

where $\alpha$ is a parameter. The discretization is carried out using J-GL-C methods. The resulting system of nonlinear equations is

$$
\begin{gathered}
\mathbf{F}(\mathbf{x})=\mathbf{S}_{x x} \mathbf{x}+\alpha e^{\mathbf{x}}=\mathbf{0}, \\
\mathbf{F}^{\prime}(\mathbf{x})=\mathbf{S}_{x x}+\alpha \operatorname{diag}\left(e^{\mathbf{x}}\right),
\end{gathered}
$$

where $\mathbf{S}_{x x}=\left(2 \mathbf{Q}_{x}\right)^{2}$. We considered several values of $\alpha$, values $\theta=-1 / 2$ and $\phi=-1 / 2$ for the parameters of J-GL-C, 50 grid points, and solved the resulting system of nonlinear equations using a single application of NMIM with varying $m$. The initial guess and computed numerical solutions are given in Figure 2. Table 6 gives the norm of the residue of the solution of the system of nonlinear equations for varying $m$.
5.2.3. Frank-Kamenetskii Problem. Frank-Kamenetskii problem is

$$
\begin{aligned}
x^{\prime \prime}(t)+\frac{1}{t} x^{\prime}(t)+\alpha e^{x(t)} & =0, \\
x^{\prime}(0) & =0, \\
x(1) & =0,
\end{aligned}
$$

where $\alpha$ is a parameter. After discretization by J-GL-C methods we get the system of nonlinear equations:

$$
\begin{gathered}
\mathbf{F}(\mathbf{x})=\mathbf{S}_{x x} \mathbf{x}+\operatorname{diag}\left(\frac{1}{\mathbf{t}}\right) \mathbf{S}_{x} \mathbf{x}+\alpha e^{\mathbf{x}}=\mathbf{0}, \\
\mathbf{F}^{\prime}(\mathbf{x})=\mathbf{S}_{x x}+\operatorname{diag}\left(\frac{1}{\mathbf{t}}\right) \mathbf{S}_{x}+\alpha \operatorname{diag}\left(e^{\mathbf{x}}\right) .
\end{gathered}
$$


TABLE 4: Verification of convergence order.

\begin{tabular}{|c|c|c|c|c|}
\hline \multicolumn{5}{|c|}{$\left\|\mathbf{F}\left(\mathbf{x}_{k}\right)\right\|_{\infty}$} \\
\hline Iter. & $m=4$ & $m=5$ & $m=6$ & $m=7$ \\
\hline \multicolumn{5}{|l|}{ Problem $1(n=4)$} \\
\hline 1 & $6.56 e-8$ & $1.09 e-11$ & $1.82 e-15$ & $3.04 e-19$ \\
\hline 2 & $6.31 e-68$ & $5.74 e-158$ & $8.66 e-286$ & $2.17 e-451$ \\
\hline 3 & $4.44 e-608$ & $6.90 e-2206$ & $6.32 e-5422$ & $6.67 e-10823$ \\
\hline $\mathrm{CCO}$ & 9 & 14 & 19 & 24 \\
\hline \multicolumn{5}{|l|}{ Problem $2(n=10)$} \\
\hline 1 & $7.31 e-3$ & $6.45 e-4$ & $5.83 e-5$ & $5.29 e-6$ \\
\hline 2 & $1.54 e-21$ & $1.38 e-47$ & $5.42 e-84$ & $8.20 e-131$ \\
\hline 3 & $1.31 e-189$ & $5.63 e-659$ & $1.35 e-1585$ & $3.06 e-3126$ \\
\hline $\mathrm{CCO}$ & 9 & 14 & 19 & 24 \\
\hline \multicolumn{5}{|l|}{ Problem $3(n=100)$} \\
\hline 1 & $7.09 e-5$ & $2.12 e-7$ & $6.36 e-10$ & $1.90 e-12$ \\
\hline 2 & $5.45 e-43$ & $1.69 e-94$ & $1.53 e-166$ & $1.64 e-258$ \\
\hline 3 & $6.87 e-348$ & $8.80 e-1143$ & $7.95 e-2673$ & $8.85 e-5184$ \\
\hline $\mathrm{CCO}$ & 8 & 12 & 16 & 20 \\
\hline \multicolumn{5}{|l|}{ Problem $4(n=5)$} \\
\hline 2 & $1.24 e-3$ & $8.47 e-6$ & $8.17 e-9$ & $9.64 e-13$ \\
\hline 3 & $2.73 e-19$ & $2.08 e-54$ & $1.45 e-120$ & $2.27 e-229$ \\
\hline 4 & $6.73 e-148$ & $1.79 e-645$ & $7.35 e-1920$ & $5.31 e-4575$ \\
\hline $\mathrm{CCO}$ & 8.21 & 12.2 & 16.1 & 20.1 \\
\hline \multicolumn{5}{|l|}{ Problem $5(n=3)$} \\
\hline 1 & $3.77 e-6$ & $8.57 e-10$ & $1.94 e-13$ & $4.39 e-17$ \\
\hline 2 & $2.90 e-67$ & $1.71 e-151$ & $6.21 e-269$ & $1.46 e-419$ \\
\hline 3 & $3.09 e-581$ & $2.79 e-1984$ & $1.71 e-4737$ & $9.99 e-9298$ \\
\hline $\mathrm{CCO}$ & 8.41 & 12.9 & 17.5 & 22.1 \\
\hline Theoretical convergence order $(5 m-11)$ & 9 & 14 & 19 & 24 \\
\hline
\end{tabular}

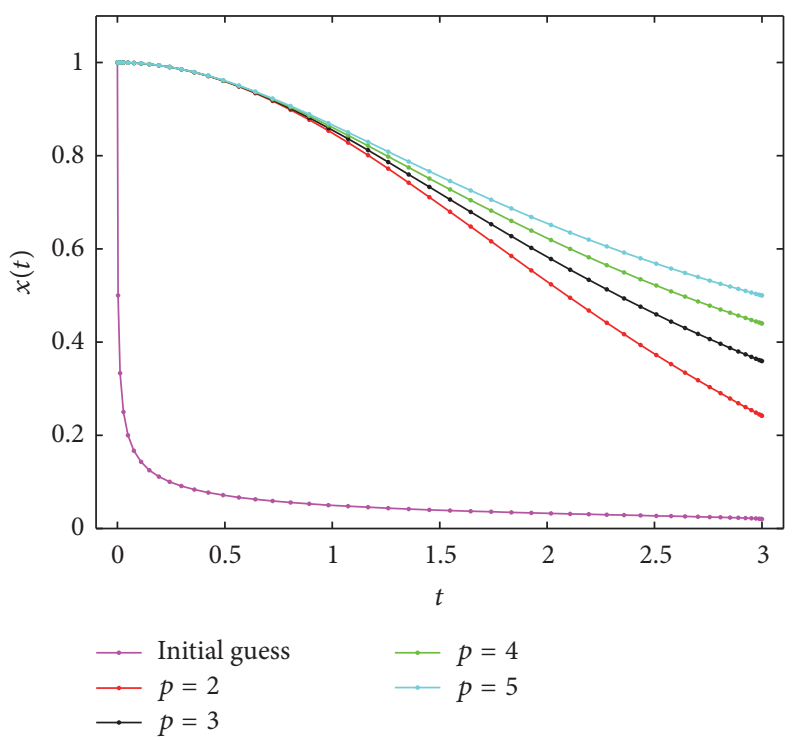

FIGURE 1: Initial guess and solutions of the Lane-Emden equation for different values of $p$. 
TABLE 5: Norm of the residue of the system of nonlinear equation resulting from Lane-Emden equation with varying $m$.

\begin{tabular}{lcccc}
\hline \multicolumn{5}{c}{$\left\|\mathbf{F}\left(\mathbf{x}_{k}\right)\right\|_{\infty}$} \\
$m$ & 2 & 3 & 4 & 5 \\
\hline 10 & $2.69 e-17$ & $3.70 e-14$ & $1.04 e-12$ & $1.03 e-11$ \\
20 & $1.36 e-46$ & $7.06 e-41$ & $9.33 e-39$ & $1.22 e-37$ \\
30 & $9.33 e-76$ & $1.68 e-67$ & $7.56 e-65$ & $1.97-63$ \\
40 & $6.16 e-105$ & $4.53 e-94$ & $6.19 e-91$ & $2.26 e-89$ \\
50 & $4.08 e-134$ & $1.21 e-120$ & $5.11 e-117$ & $2.18 e-115$ \\
60 & $2.71 e-163$ & $3.22 e-147$ & $4.24 e-143$ & $2.00 e-141$ \\
70 & $1.79 e-192$ & $8.555 e-174$ & $3.52 e-169$ & $1.83 e-167$ \\
80 & $1.19 e-221$ & $2.27 e-200$ & $2.92 e-195$ & $1.69 e-193$ \\
90 & $7.89 e-251$ & $6.03 e-227$ & $2.43 e-221$ & $1.58 e-219$ \\
100 & $5.23 e-280$ & $1.60 e-253$ & $2.02 e-247$ & $1.47 e-245$ \\
\hline
\end{tabular}

TABLE 6: Norm of the residue of the solution of the system of nonlinear equations for Bratu problem and varying $m$.

\begin{tabular}{lccc}
\hline \multicolumn{3}{c}{$\left\|\mathbf{F}\left(\mathbf{x}_{k}\right)\right\|_{\infty}$} \\
$m$ & 1 & 2 & 3 \\
\hline 10 & $7.68 e-41$ & $4.10 e-31$ & $1.97 e-28$ \\
20 & $2.20 e-93$ & $1.01 e-70$ & $9.65 e-64$ \\
30 & $7.02 e-146$ & $2.50 e-110$ & $4.72 e-99$ \\
40 & $2.23 e-198$ & $6.17 e-150$ & $2.30 e-134$ \\
50 & $7.13 e-251$ & $1.52 e-189$ & $1.12 e-169$ \\
60 & $2.27 e-303$ & $3.75 e-229$ & $5.52 e-205$ \\
70 & $7.30 e-356$ & $9.27 e-269$ & $2.69 e-240$ \\
80 & $2.30 e-408$ & $2.28 e-308$ & $1.32 e-275$ \\
90 & $7.40 e-461$ & $5.70 e-348$ & $6.50 e-311$ \\
100 & $2.30 e-513$ & $1.40 e-387$ & $3.20 e-346$ \\
\hline
\end{tabular}

TABLE 7: Norm of the residue of the solution of the system of nonlinear equations for Fran-Kamenetskii problem and varying $m$.

\begin{tabular}{lcccc}
\hline \multicolumn{5}{c}{$\left\|\mathrm{F}\left(\mathbf{x}_{k}\right)\right\|_{\infty}$} \\
$m$ & 1 & 1.1 & 1.2 & 1.3 \\
\hline 10 & $7.09 e-41$ & $1.89 e-39$ & $2.63 e-38$ & $1.80 e-37$ \\
20 & $2.46 e-82$ & $2.36 e-79$ & $6.21 e-77$ & $4.05 e-75$ \\
30 & $8.58 e-124$ & $2.95 e-119$ & $1.46 e-115$ & $9.09 e-113$ \\
40 & $2.98 e-165$ & $3.69 e-159$ & $3.45 e-154$ & $2.03 e-150$ \\
50 & $1.03 e-206$ & $4.61 e-199$ & $8.15 e-193$ & $4.57 e-188$ \\
60 & $3.61 e-248$ & $5.76 e-239$ & $1.92 e-231$ & $1.02 e-225$ \\
70 & $1.25 e-289$ & $7.19 e-279$ & $4.53 e-270$ & $2.29 e-263$ \\
80 & $4.40 e-331$ & $9.00 e-319$ & $1.10 e-308$ & $5.15 e-301$ \\
90 & $1.50 e-372$ & $1.10 e-358$ & $2.50 e-347$ & $1.20 e-338$ \\
100 & $5.30 e-414$ & $1.40 e-398$ & $6.00 e-386$ & $2.60 e-376$ \\
\hline
\end{tabular}

We considered several values of $\alpha$, values $\theta=-1 / 2$ and $\phi=-1 / 2$ for the parameters of J-GL-C, 50 grid points, and solved the resulting system of nonlinear equations by using a single application of NMIM with varying $m$. Figure 3 plots the initial guess and computed solutions and Table 7 gives the

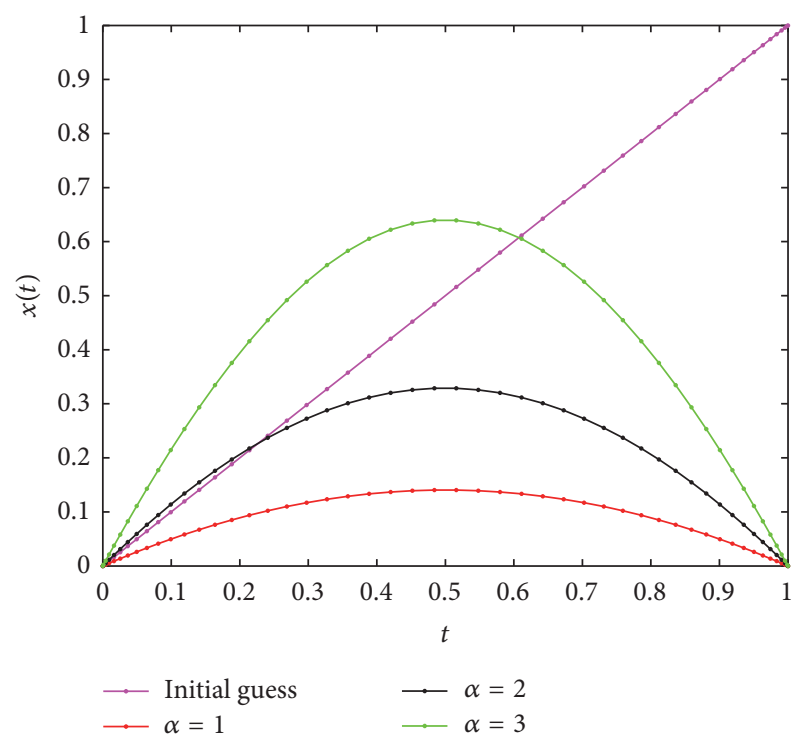

FIGURE 2: Initial guess and solutions of Bratu problem for different values of $\alpha$.

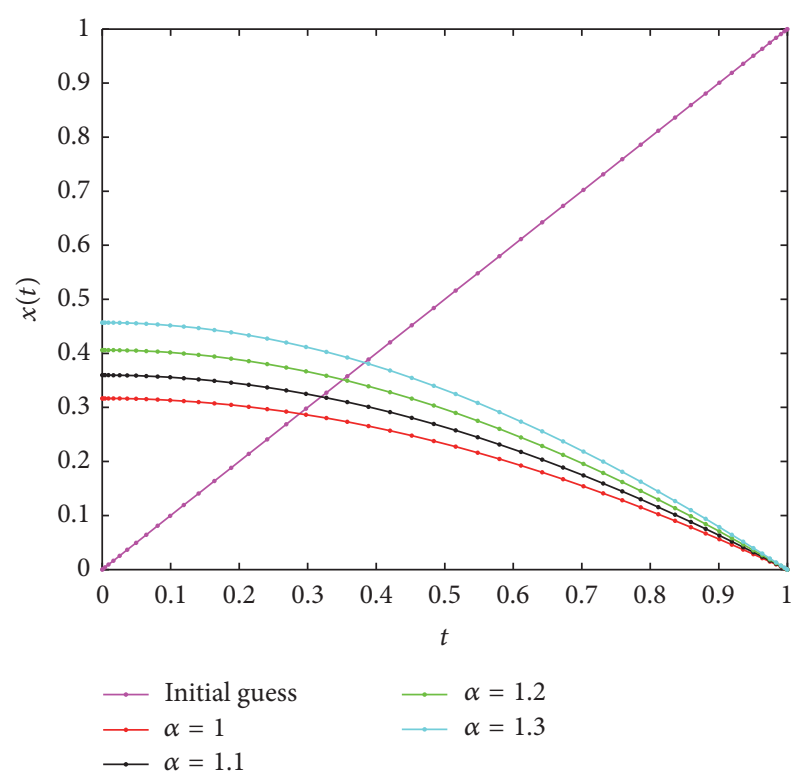

FIGURE 3: Initial guess and solutions of Frank-Kamenetskii problem with different values of $\alpha$.

norm of the residue of the solution of the system of nonlinear equations.

5.2.4. $1+1$ Nonlinear Schrödinger Equation. $1+1$ nonlinear Schrödinger equation is

$$
\begin{aligned}
& i \partial_{t} \psi(x, t)+\partial_{x x} \psi(x, t)+2 \gamma|\psi(x, t)|^{2} \psi(x, t) \\
& -2 \delta R(x, t) \psi(x, t)=0, \quad(x, t) \in D_{x} \times D_{t},
\end{aligned}
$$


TABLE 8: Nonlinear Schrödinger equations.

Schrödinger equation

$i \partial_{t} \psi+\partial_{x x} \psi+2|\psi|^{2} \psi=0$

$i \partial_{t} \psi+\partial_{x x} \psi-2|\psi|^{2} \psi=0$

$i \partial_{t} \psi+\frac{1}{2} \partial_{x x} \psi-|\psi|^{2} \psi-\cos (x)^{2} \psi=0$

$i \partial_{t} \psi+\partial_{x x} \psi+2|\psi|^{2} \psi-2 \delta \psi=0$

Analytical Solution
$\psi(x, t)=e^{i(x+t)}$
$\psi(x, t)=e^{i(x-3 t)}$
$\psi(x, t)=e^{-3 i t / 2} \sin (x)$
$\psi(x, t)=e^{i(x+(1-2 \delta) t)}$

where

$$
\begin{aligned}
& D_{x}=\left[a_{x}, b_{x}\right], \\
& D_{t}=\left[0, t_{f}\right],
\end{aligned}
$$

with the initial and boundary conditions

$$
\begin{aligned}
& \psi\left(a_{x}, t\right)=\eta_{1}(t), \\
& \psi\left(b_{x}, t\right)=\eta_{2}(t), \\
& \psi(x, 0)=\zeta(x) .
\end{aligned}
$$

The function $\psi(x, t)$ is a complex function and can be written as $\psi(x, t)=\psi_{1}(x, t)+i \psi_{2}(x, t)$. The Schrödinger equation can be rewritten in terms of the real functions $\psi_{1}(x, t)$ and $\psi_{2}(x, t)$ as

$$
\begin{gathered}
{\left[\begin{array}{cc}
\partial_{t} & \partial_{x x} \\
\partial_{x x} & -\partial_{t}
\end{array}\right]\left[\begin{array}{l}
\psi_{1} \\
\psi_{2}
\end{array}\right]-2 \delta R(x, t)\left[\begin{array}{ll}
0 & 1 \\
1 & 0
\end{array}\right]\left[\begin{array}{l}
\psi_{1} \\
\psi_{2}
\end{array}\right]} \\
+2 \gamma\left(\psi_{1}^{2}+\psi_{2}^{2}\right)\left[\begin{array}{ll}
0 & 1 \\
1 & 0
\end{array}\right]\left[\begin{array}{l}
\psi_{1} \\
\psi_{2}
\end{array}\right]=0,
\end{gathered}
$$

with initial and boundary conditions

$$
\begin{aligned}
& \psi_{1}\left(a_{x}, t\right)+i \psi_{2}\left(a_{x}, t\right)=\eta_{11}(t)+i \eta_{12}, \\
& \psi_{1}\left(b_{x}, t\right)+i \psi_{2}\left(b_{x}, t\right)=\eta_{21}(t)+i \eta_{22}(t), \\
& \psi_{1}(x, 0)+i \psi_{2}(x, 0)=\zeta_{1}(x)+i \zeta_{2}(x) .
\end{aligned}
$$

Using J-GL-C methods, we can discretize (30) as

$$
\begin{array}{r}
\mathbf{F}(\boldsymbol{\psi})=\left[\begin{array}{cc}
\mathbf{S}_{t} \otimes \mathbf{I}_{x} & \mathbf{I}_{t} \otimes \mathbf{S}_{x x} \\
\mathbf{I}_{t} \otimes \mathbf{S}_{x x} & -\mathbf{S}_{t} \otimes \mathbf{I}_{x}
\end{array}\right]\left[\begin{array}{l}
\psi_{1} \\
\psi_{2}
\end{array}\right] \\
-2 \delta\left[\begin{array}{cc}
\mathbf{O} & \operatorname{diag}(\mathbf{R}) \\
\operatorname{diag}(\mathbf{R}) & \mathbf{O}
\end{array}\right]\left[\begin{array}{l}
\psi_{1} \\
\psi_{2}
\end{array}\right]
\end{array}
$$

where $\psi_{j}=\left[\psi_{j_{(1,1)}}, \psi_{j_{(1,2)}}, \ldots, \psi_{j_{\left(1, n_{t}\right)}}, \ldots, \psi_{j_{\left(n_{x}, 1\right)}}, \psi_{\left.j_{\left(n_{x}, 2\right)}\right)}\right.$,

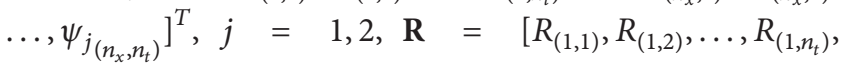
$\left.\ldots, R_{\left(n_{x}, 1\right)}, R_{\left(n_{x}, 2\right)}, \ldots, R_{\left(n_{x}, n_{t}\right)}\right]^{T}, \otimes$ is the Kronecker product, $\odot$ is the element-wise multiplication, $\mathbf{O}$ is a matrix of all zeros, $\mathbf{I}$ is the identity matrix, $\mathbf{S}_{t}=\left(2 / t_{f}\right) \mathbf{Q}_{t}, \mathbf{S}_{x x}=\left(\left(2 /\left(b_{x}-\right.\right.\right.$ $\left.\left.\left.a_{x}\right)\right) \mathbf{Q}_{x}\right)^{2}$, and $\mathbf{Q}$ is the J-GL-C operational matrix. The system of nonlinear equations (32) can be rewritten as

$$
\mathbf{F}(\psi)=\mathbf{A} \psi+g(\psi)-\mathbf{p},
$$

where $\mathbf{A}=\left[\begin{array}{cc}\mathbf{S}_{t} \otimes \mathbf{I}_{x} & \mathbf{I}_{\boldsymbol{t}} \otimes \mathbf{S}_{x x} \\ \mathbf{I}_{t} \otimes \mathbf{S}_{x x}-\mathbf{S}_{t} \otimes \mathbf{I}_{x}\end{array}\right]-2 \delta\left[\begin{array}{cc}\mathbf{0} & \operatorname{diag}(\mathbf{R}) \\ \operatorname{diag}(\mathbf{R}) & \mathbf{O}\end{array}\right], g(\psi)=$ $2 \gamma\left[\begin{array}{cc}2 \operatorname{diag}\left(\psi_{1} \odot \psi_{2}\right) & 3 \operatorname{diag}\left(\psi_{2}^{2}\right) \\ 3 \operatorname{diag}\left(\psi_{1}^{2}\right) & 2 \operatorname{diag}\left(\psi_{1} \odot \psi_{2}\right)\end{array}\right]$, and $\mathbf{p}$ is the vector incorporating the initial-boundary conditions. Four nonlinear Schrödinger equations are listed in Table 8 with their corresponding analytical solutions. We chose a domain $(x, t) \epsilon$ $[-1,1] \times[0,1]$ and solved the equations using different pair of values for the parameters $\theta$ and $\phi$ of J-GL-C and different grids for the domain. As initial guess, we used $\psi_{1}=\mathbf{0}, \psi_{2}=\mathbf{0}$ with boundary conditions set for problem 3. For problems 1,2 , and 4 NMIM does not converge with that initial guess and we made the initial guess smoother. We smoothed the initial guess by applying the iteration

$$
\psi=-\mathbf{A}^{-1}(2 g(\psi)-\mathbf{p})
$$

once for problem 1 and twice for problems 2 and 4 . Tables 9 , 10,11 , and 12 give the norm of the error in the solution when 
TABLE 9: Norm of the error in the solution for equation (17).

\begin{tabular}{|c|c|c|c|c|}
\hline & $\| \psi-\psi_{\text {analyt }}$ & & & \\
\hline$m$ & & & Grid size & \\
\hline & & $5 \times 30$ & $10 \times 30$ & $15 \times 30$ \\
\hline 1 & & $5.49 e-01$ & $6.21 e-01$ & $6.41 e-01$ \\
\hline 4 & & $5.36 e-02$ & $2.35 e-01$ & $2.44 e-01$ \\
\hline 8 & Jeoendre nolynomials $(\theta-\phi)-(0-0)$ & $2.85 e-04$ & $4.55 e-04$ & $4.97 e-04$ \\
\hline 12 & Legentire porynominds $(0, \varphi)-(0,0)$ & $2.85 e-04$ & $5.81 e-08$ & $6.38 e-08$ \\
\hline 16 & & $2.85 e-04$ & $5.69 e-10$ & $4.38 e-12$ \\
\hline 20 & & $2.85 e-04$ & $6.13 e-11$ & $5.95 e-13$ \\
\hline 1 & & $6.86 e-01$ & & $6.40 e-01$ \\
\hline 4 & & $1.96 e-01$ & & $2.48 e-01$ \\
\hline 8 & $(\theta, \phi)=(1 / 2,0)$ & $1.64 e-03$ & Divergent & $4.94 e-04$ \\
\hline 12 & & $1.59 e-03$ & & $6.16 e-08$ \\
\hline 16 & & $1.59 e-03$ & & $6.02 e-12$ \\
\hline 20 & & $1.59 e-03$ & & $2.16 e-13$ \\
\hline 1 & & $5.24 e-01$ & $6.25 e-01$ & $6.41 e-01$ \\
\hline 4 & & $5.90 e-02$ & $2.39 e-01$ & $2.46 e-01$ \\
\hline 8 & Chebyshev polynomials of second kind $(\theta, \phi)=(1 / 2,1 / 2)$ & $1.14 e-03$ & $5.05 e-04$ & $5.40 e-04$ \\
\hline 12 & 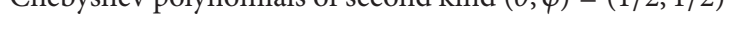 & $1.14 e-03$ & $7.73 e-08$ & $8.00 e-08$ \\
\hline 16 & & $1.14 e-03$ & $1.90 e-09$ & $5.28 e-12$ \\
\hline 20 & & $1.14 e-03$ & $1.90 e-09$ & $4.99 e-13$ \\
\hline 1 & & $5.95 e-01$ & $6.20 e-01$ & $6.41 e-01$ \\
\hline 4 & & $3.73 e-02$ & $2.37 e-01$ & $2.45 e-01$ \\
\hline 8 & Chebyshev polynomials of first kind $(\theta, \phi)=(-1 / 2,-1 / 2)$ & $1.52 e-03$ & $4.24 e-04$ & $4.81 e-04$ \\
\hline 12 & & $1.52 e-03$ & $5.22 e-08$ & $5.81 e-08$ \\
\hline 16 & & $1.52 e-03$ & $4.90 e-10$ & $1.71 e-12$ \\
\hline 20 & & $1.52 e-03$ & $4.90 e-10$ & $9.31 e-14$ \\
\hline
\end{tabular}

the nonlinear system of equations is solved using a single application of NMIM with varying $m$. Figures 4-7 display the solutions and the error in the components of $\psi_{1}$ and $\psi_{2}$ for $\theta=-4 / 2, \phi=-1 / 2$, and a grid $15 \times 30$.

\subsubsection{Klein Gordon Equation. Klein Gordon equation is}

$$
\begin{aligned}
u_{t t}(x, t)-c^{2} u_{x x}(x, t)+k u-\gamma u(x, t)^{3} & =0, \\
(x, t) & \in D_{x} \times D_{t},
\end{aligned}
$$

where

$$
\begin{aligned}
D_{x} & =\left[a_{x}, b_{x}\right], \\
D_{t} & =\left[0, t_{f}\right],
\end{aligned}
$$

with the initial and boundary conditions

$$
\begin{aligned}
& u\left(a_{x}, t\right)=\varrho_{1}(t), \\
& u\left(b_{x}, t\right)=\varrho_{2}(t),
\end{aligned}
$$

$$
\begin{aligned}
u(x, 0) & =\xi_{1}(x), \\
u_{t}(x, 0) & =\xi_{2}(x) .
\end{aligned}
$$

By discretization using J-GL-C methods we obtain

$$
\begin{gathered}
\mathbf{F}(\mathbf{u})=\mathbf{S}_{t t} \mathbf{u}-c^{2} \mathbf{S}_{x x} \mathbf{u}+k \mathbf{u}-\gamma \mathbf{u}^{3}=\mathbf{0}, \\
\mathbf{F}^{\prime}(\mathbf{u})=\mathbf{S}_{t t}-c^{2} \mathbf{S}_{x x}+k \mathbf{I}-3 \gamma \operatorname{diag}\left(\mathbf{u}^{2}\right),
\end{gathered}
$$

where $\mathbf{u}=\left[u_{(1,1)}, u_{(1,2)}, \ldots, u_{\left(1, n_{t}\right)}, \ldots, u_{\left(n_{x}, 1\right)}, u_{\left(n_{x}, 2\right)}, \ldots\right.$, $\left.u_{\left(n_{x}, n_{t}\right)}\right]^{T}, \mathbf{S}_{t t}=\left(\left(2 / t_{f}\right) \mathbf{Q}_{t}\right)^{2}$, and $\mathbf{S}_{x x}=\left(\left(2 /\left(b_{x}-a_{x}\right)\right) \mathbf{Q}_{x}\right)^{2}$. The analytical solution of $(35)$ is $u(x, t)=\delta \operatorname{sech}(\kappa(x-v t))$, where $\kappa=\sqrt{k /\left(c^{2}-v^{2}\right)}$ and $\delta=\sqrt{2 k / \gamma}$. We set the parameters $c=1, \gamma=1, v=0.5$, and $k=0.5$, picked up the domain $(x, t) \in[-10,10] \times[0,1]$, and solved the nonlinear system of equations by a single application of NMIM with initial guess $\mathbf{u}=\mathbf{0}$, with the boundary conditions introduced. We used several pairs of the $\theta, \phi$ parameters of J-GL-C. According to Table 13, the Chebyshev collocation method of first kind exhibits best accuracy when the grid is more refined. The 
TABLE 10: Norm of the error in the solution for equation (30).

\begin{tabular}{|c|c|c|c|c|}
\hline \multicolumn{5}{|c|}{$\left\|\psi-\psi_{\text {analytical }}\right\|_{\infty}$} \\
\hline$m$ & & & Grid size & \\
\hline & & $5 \times 30$ & $10 \times 30$ & $15 \times 30$ \\
\hline 1 & \multirow{6}{*}{ Legendre polynomials $(\theta, \phi)=(0,0)$} & $1.28 e+00$ & $1.34 e+00$ & $1.34 e+00$ \\
\hline 4 & & $4.27 e-01$ & $9.65 e-01$ & $9.72 e-01$ \\
\hline 8 & & $1.25 e-03$ & $2.15 e-03$ & $2.18 e-03$ \\
\hline 12 & & $1.46 e-04$ & $9.17 e-07$ & $1.05 e-06$ \\
\hline 16 & & $1.46 e-04$ & $3.32 e-10$ & $4.61 e-10$ \\
\hline 20 & & $1.46 e-04$ & $2.90 e-11$ & $1.85 e-13$ \\
\hline 1 & \multirow{6}{*}{$(\theta, \phi)=(1 / 2,0)$} & $1.27 e+00$ & $1.35 e+00$ & $1.34 e+00$ \\
\hline 4 & & $4.34 e-01$ & $9.46 e-01$ & $9.72 e-01$ \\
\hline 8 & & $1.63 e-03$ & $2.18 e-03$ & $2.18 e-03$ \\
\hline 12 & & $1.52 e-03$ & $3.89 e-07$ & $8.77 e-07$ \\
\hline 16 & & $1.52 e-03$ & $5.28 e-09$ & $1.95 e-10$ \\
\hline 20 & & $1.52 e-03$ & $3.73 e-09$ & $1.34 e-13$ \\
\hline 1 & \multirow{6}{*}{ Chebyshev polynomials of second kind $(\theta, \phi)=(1 / 2,1 / 2)$} & $1.26 e+00$ & $1.34 e+00$ & $1.34 e+00$ \\
\hline 4 & & $3.98 e-01$ & $9.24 e-01$ & $9.40 e-01$ \\
\hline 8 & & $2.63 e-03$ & $1.35 e-03$ & $1.63 e-03$ \\
\hline 12 & & $1.00 e-03$ & $2.63 e-06$ & $2.85 e-06$ \\
\hline 16 & & $1.00 e-03$ & $2.35 e-08$ & $1.65 e-08$ \\
\hline 20 & & $1.00 e-03$ & $7.88 e-10$ & $6.29 e-11$ \\
\hline 1 & \multirow{6}{*}{ Chebyshev polynomials of first kind $(\theta, \phi)=(-1 / 2,-1 / 2)$} & $1.33 e+00$ & $1.34 e+00$ & $1.34 e+00$ \\
\hline 4 & & $3.90 e-01$ & $9.80 e-01$ & $9.80 e-01$ \\
\hline 8 & & $1.03 e-03$ & $2.15 e-03$ & $2.19 e-03$ \\
\hline 12 & & $1.03 e-03$ & $6.56 e-07$ & $7.42 e-07$ \\
\hline 16 & & $1.03 e-03$ & $3.08 e-10$ & $1.57 e-10$ \\
\hline 20 & & $1.03 e-03$ & $2.95 e-10$ & $5.77 e-14$ \\
\hline
\end{tabular}
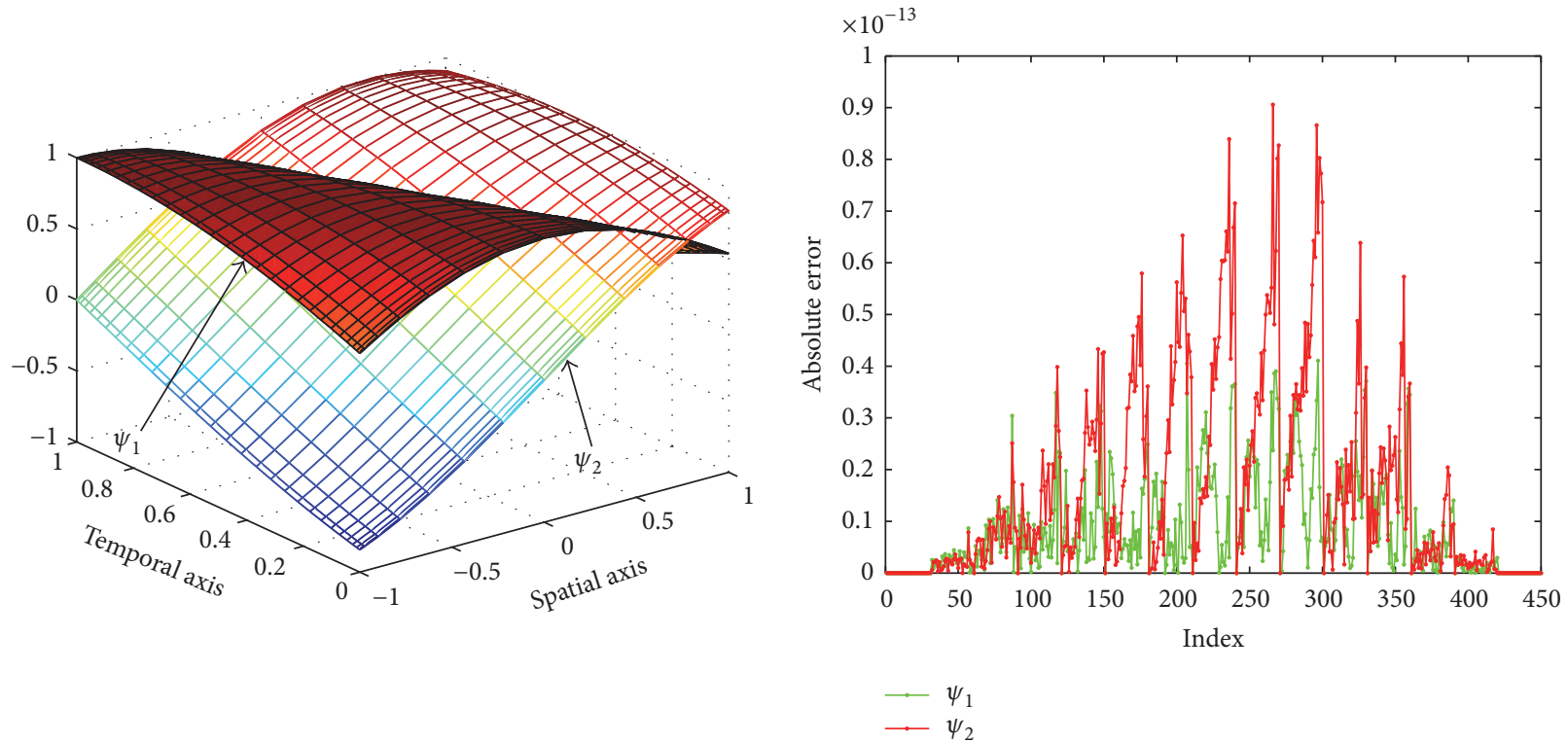

FIgURE 4: Solution and error components for (17). 
TABLE 11: Norm of the error in the solution for equation (32).

\begin{tabular}{|c|c|c|c|c|}
\hline & $\| \psi-\psi_{\text {analy }}$ & & & \\
\hline$m$ & & & Grid size & \\
\hline$m$ & & $5 \times 30$ & $10 \times 30$ & $15 \times 30$ \\
\hline 1 & & $9.98 e-02$ & $8.73 e-02$ & $9.66 e-02$ \\
\hline 4 & & $7.62 e-04$ & $3.16 e-04$ & $3.25 e-04$ \\
\hline 8 & Legendre polynomial $(\theta, \phi)=(0,0)$ & $4.27 e-05$ & $3.64 e-11$ & $3.12 e-11$ \\
\hline 12 & Legenare porynomial $(\theta, \varphi)=(0,0)$ & $4.27 e-05$ & $3.16 e-11$ & $1.89 e-13$ \\
\hline 16 & & $4.27 e-05$ & $3.16 e-11$ & $1.88 e-13$ \\
\hline 20 & & $4.27 e-05$ & $3.16 e-11$ & $1.89 e-13$ \\
\hline 1 & & $9.71 e-02$ & $9.33 e-02$ & $9.67 e-02$ \\
\hline 4 & & $1.18 e-03$ & $3.37 e-04$ & $3.36 e-04$ \\
\hline 8 & $(\theta, \phi)=(1 / 2,0)$ & $7.68 e-04$ & $3.23 e-08$ & $2.93 e-11$ \\
\hline 12 & & $7.68 e-04$ & $1.89 e-10$ & $1.07 e-13$ \\
\hline 16 & & $7.68 e-04$ & $1.87 e-10$ & $1.06 e-13$ \\
\hline 20 & & $7.68 e-04$ & $1.87 e-10$ & $1.07 e-13$ \\
\hline 1 & & $9.47 e-02$ & $9.17 e-02$ & $9.65 e-02$ \\
\hline 4 & & $9.17 e-04$ & $3.14 e-04$ & $3.38 e-04$ \\
\hline 8 & Chebvshey polvnomials of second kind $(\theta, \phi)=(1 / 2,1 / 2)$ & $6.70 e-04$ & $9.13 e-11$ & $8.39 e-11$ \\
\hline 12 & 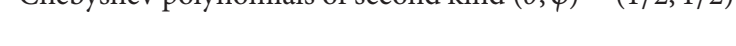 & $6.70 e-04$ & $8.92 e-11$ & $1.19 e-13$ \\
\hline 16 & & $6.70 e-04$ & $8.92 e-11$ & $1.19 e-13$ \\
\hline 20 & & $6.70 e-04$ & $8.92 e-11$ & $1.19 e-13$ \\
\hline 1 & & $1.01 e-01$ & $9.01 e-02$ & $9.61 e-02$ \\
\hline 4 & & $1.31 e-03$ & $2.99 e-04$ & $3.26 e-04$ \\
\hline 8 & Chebyshev polynomial of first kind $(\theta, \phi)=(-1 / 2,-1 / 2)$ & $8.53 e-04$ & $4.90 e-11$ & $2.27 e-11$ \\
\hline 12 & 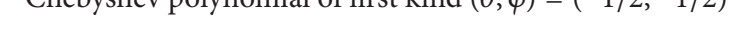 & $8.53 e-04$ & $5.59 e-11$ & $3.28 e-14$ \\
\hline 16 & & $8.53 e-04$ & $5.59 e-11$ & $3.24 e-14$ \\
\hline 20 & & $8.53 e-04$ & $5.59 e-11$ & $3.20 e-14$ \\
\hline
\end{tabular}
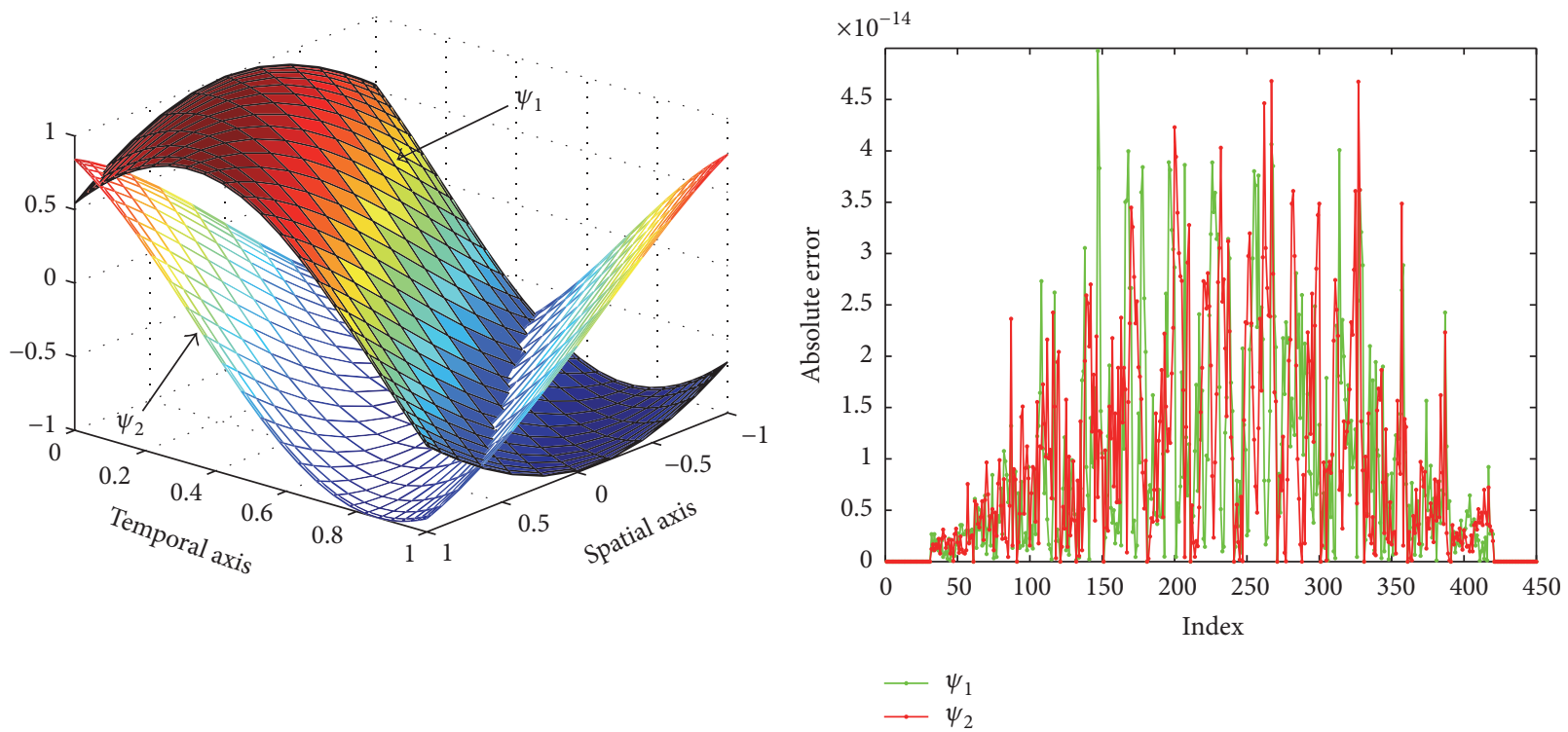

FIGURE 5: Solution and error components for (30). 
TABLE 12: Norm of the error in the solution for equation (35).

\begin{tabular}{|c|c|c|c|c|}
\hline \multicolumn{5}{|c|}{$\left\|\psi-\psi_{\text {analytical }}\right\|_{\infty}$} \\
\hline \multirow{2}{*}{$m$} & & \multicolumn{3}{|c|}{ Grid size } \\
\hline & & $5 \times 30$ & $10 \times 30$ & $15 \times 30$ \\
\hline 1 & \multirow{6}{*}{ Legendre polynomials $(\theta, \phi)=(0,0)$} & $6.44 e-01$ & $7.84 e-01$ & $8.01 e-01$ \\
\hline 4 & & $6.52 e-02$ & $4.18 e-02$ & $4.18 e-02$ \\
\hline 8 & & $2.77 e-04$ & $1.36 e-06$ & $1.33 e-06$ \\
\hline 12 & & $2.75 e-04$ & $1.70 e-10$ & $1.81 e-10$ \\
\hline 16 & & $2.75 e-04$ & $4.14 e-11$ & $4.87 e-13$ \\
\hline 20 & & $2.75 e-04$ & $4.14 e-11$ & $4.67 e-13$ \\
\hline 1 & \multirow{6}{*}{$(\theta, \phi)=(1 / 2,0)$} & $6.51 e-01$ & \multirow{6}{*}{ Divergent } & $8.08 e-01$ \\
\hline 4 & & $4.52 e-02$ & & $4.15 e-02$ \\
\hline 8 & & $1.50 e-03$ & & $1.25 e-06$ \\
\hline 12 & & $1.51 e-03$ & & $1.17 e-10$ \\
\hline 16 & & $1.51 e-03$ & & $1.94 e-13$ \\
\hline 20 & & $1.51 e-03$ & & $1.99 e-13$ \\
\hline 1 & \multirow{6}{*}{ Chebyshev polynomials of second kind $(\theta, \phi)=(1 / 2,1 / 2)$} & $7.48 e-01$ & $7.85 e-01$ & $8.04 e-01$ \\
\hline 4 & & $5.30 e-02$ & $4.24 e-02$ & $4.22 e-02$ \\
\hline 8 & & $1.02 e-03$ & $2.20 e-06$ & $2.61 e-06$ \\
\hline 12 & & $1.02 e-03$ & $2.67 e-09$ & $1.00 e-09$ \\
\hline 16 & & $1.02 e-03$ & $2.25 e-09$ & $4.86 e-13$ \\
\hline 20 & & $1.02 e-03$ & $2.25 e-09$ & $1.96 e-13$ \\
\hline 1 & \multirow{6}{*}{ Chebyshev polynomials of first kind $(\theta, \phi)=(-1 / 2,-1 / 2)$} & $7.03 e-01$ & $7.88 e-01$ & $8.02 e-01$ \\
\hline 4 & & $3.66 e-02$ & $4.20 e-02$ & $4.17 e-02$ \\
\hline 8 & & $1.46 e-03$ & $1.27 e-06$ & $1.18 e-06$ \\
\hline 12 & & $1.46 e-03$ & $5.96 e-10$ & $2.39 e-11$ \\
\hline 16 & & $1.46 e-03$ & $5.84 e-10$ & $8.56 e-14$ \\
\hline 20 & & $1.46 e-03$ & $5.84 e-10$ & $8.49 e-14$ \\
\hline
\end{tabular}
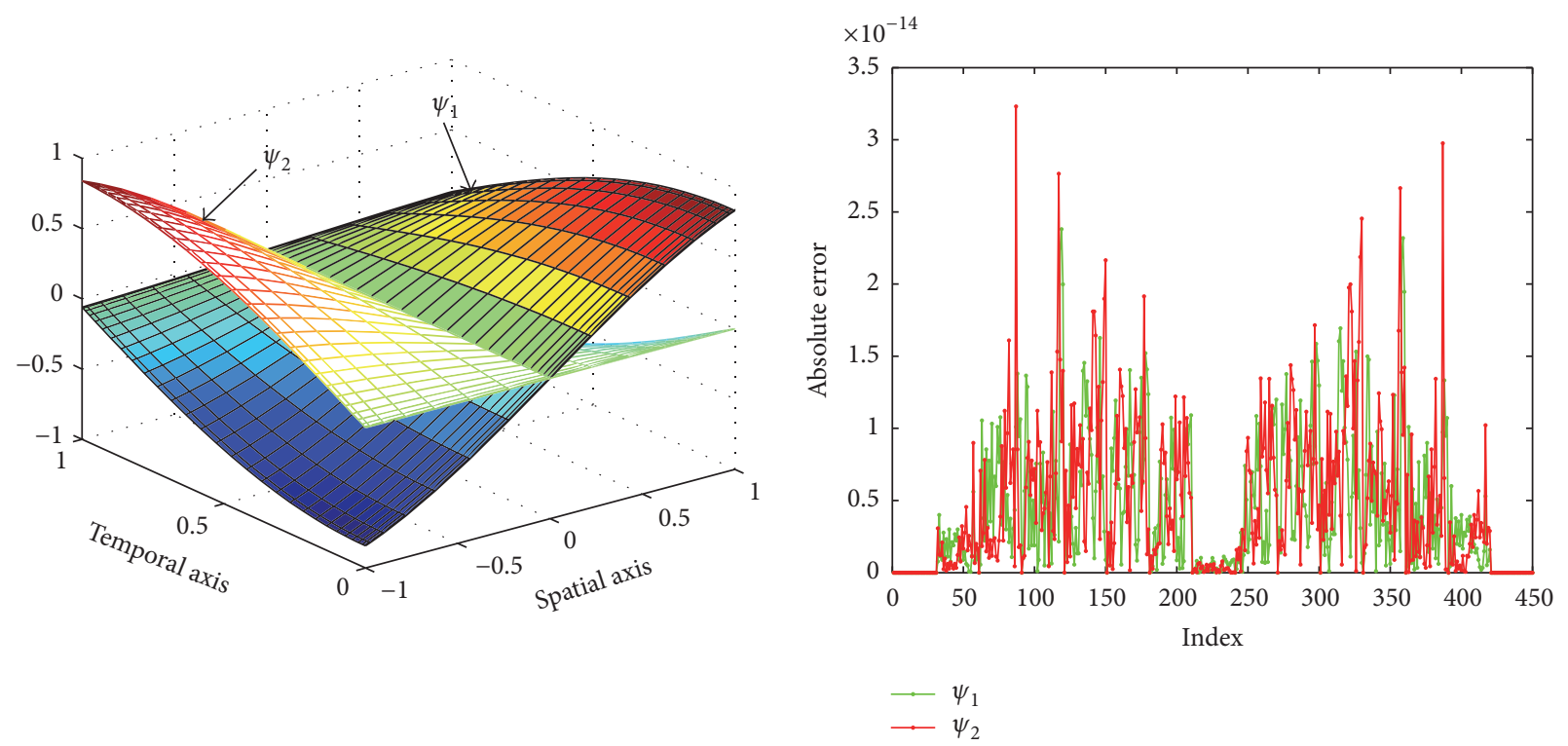

FIGURE 6: Solution and error components for (32). 

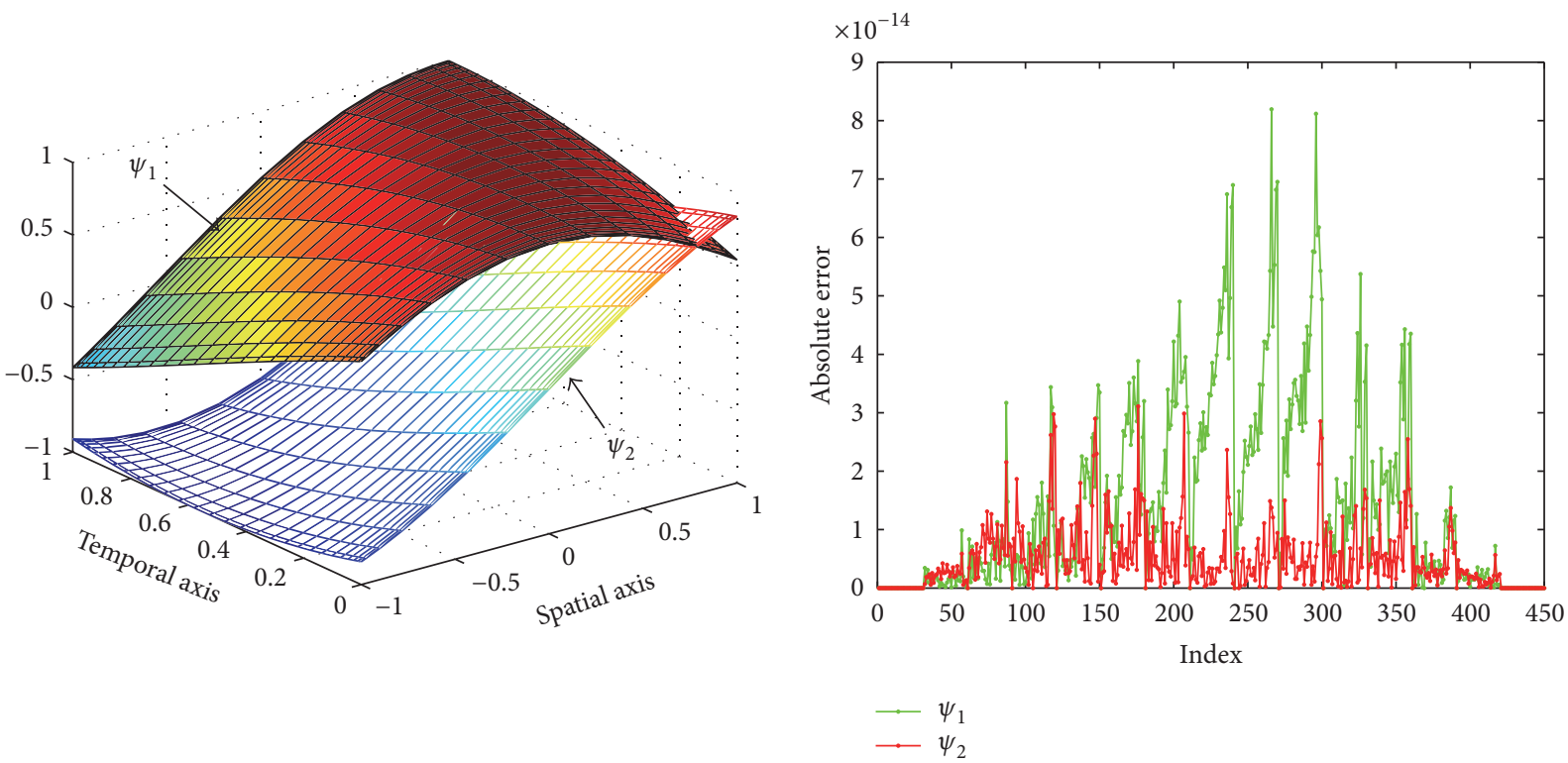

FIGURE 7: Solution and error components for (35).
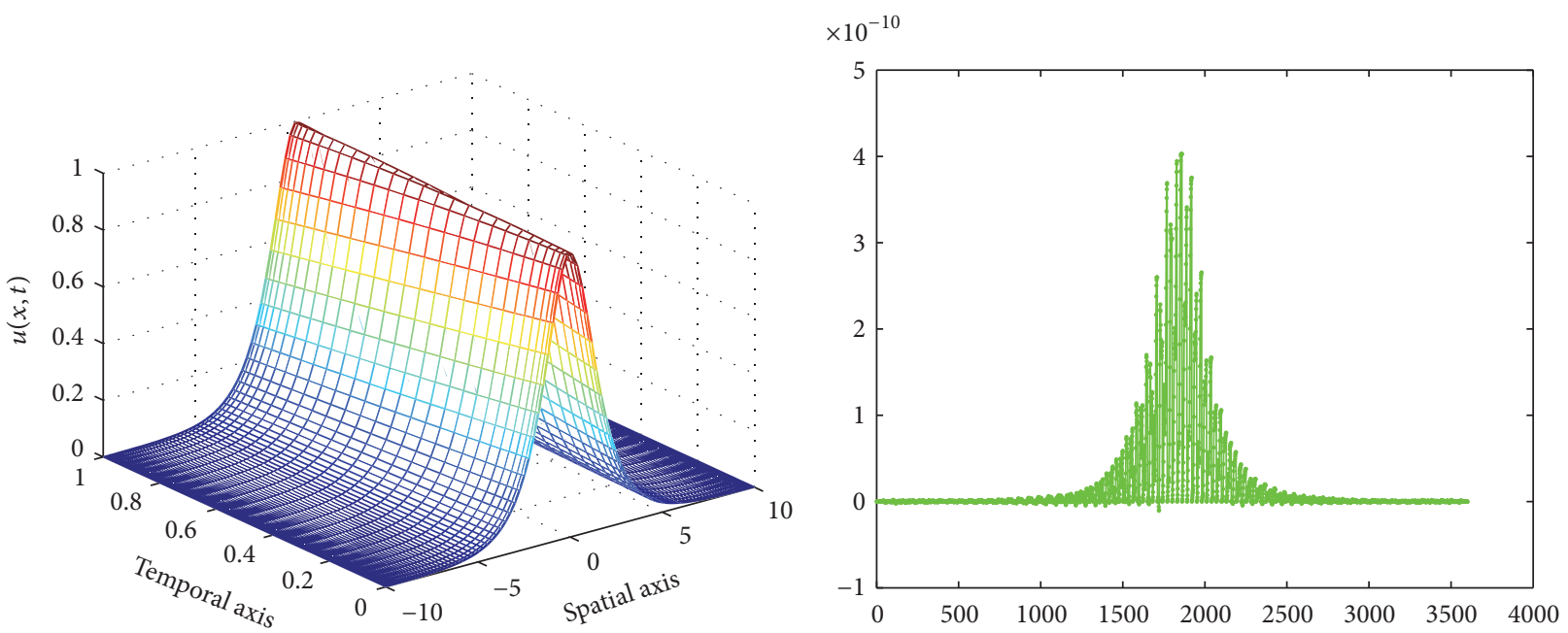

FIGURE 8: Solution and error components for nonlinear Klein Gordon equation.

solution and the error components for $\theta=-1 / 2, \phi=-1 / 2$, $n_{x}=120$, and $n_{t}=20$ are plotted in Figure 8.

5.2.6. 2D Nonlinear Wave Equation. The $2 \mathrm{D}$ nonlinear wave equation is

$$
\begin{gathered}
u_{t t}(x, y, t)-c^{2}\left(u_{x x}(x, y, t)+u_{y y}(x, y, t)\right)+f(u) \\
=p(t, x, y), \quad(x, y, t) \in D_{x} \times D_{y} \times D_{t},
\end{gathered}
$$

where

$$
\begin{aligned}
D_{x} & =\left[a_{x}, b_{x}\right], \\
D_{y} & =\left[a_{y}, b_{y}\right], \\
D_{t} & =\left[0, t_{f}\right],
\end{aligned}
$$

with the initial and boundary conditions

$$
\begin{aligned}
& u\left(a_{x}, y, t\right)=\varrho_{11}(y, t), \\
& u\left(b_{x}, y, t\right)=\varrho_{12}(y, t), \\
& u\left(x, a_{y}, t\right)=\varrho_{21}(x, t), \\
& u\left(x, b_{y}, t\right)=\varrho_{22}(x, t), \\
& u(x, y, 0)=\zeta(x, y) .
\end{aligned}
$$


TABLE 13: Norm of the error in the Klein Gordon equation as a function of $m$ for several values for $\theta$ and $\phi$ and grids.

\begin{tabular}{|c|c|c|c|c|}
\hline & $\| \mathbf{u}-\mathbf{u}_{\text {analyt }}$ & & & \\
\hline$m$ & & & Grid size & \\
\hline$m$ & & $30 \times 30$ & $60 \times 30$ & $120 \times 30$ \\
\hline 1 & & $3.80 e-01$ & $4.10 e-01$ & $4.09 e-01$ \\
\hline 2 & & $7.37 e-02$ & $8.39 e-02$ & $8.34 e-02$ \\
\hline 3 & Jerendre nolynomials $(\theta-\phi)-(0,0)$ & $1.18 e-02$ & $8.73 e-03$ & $8.65 e-03$ \\
\hline 4 & Legenare porynomials $(\theta, \phi)=(0,0)$ & $8.53 e-03$ & $1.42 e-05$ & $1.40 e-06$ \\
\hline 5 & & $8.53 e-03$ & $1.42 e-05$ & $4.37 e-09$ \\
\hline 6 & & $8.53 e-03$ & $1.42 e-05$ & $4.56 e-09$ \\
\hline 1 & & $3.98 e-01$ & $4.10 e-01$ & $4.07 e-01$ \\
\hline 2 & & $7.64 e-02$ & $8.40 e-02$ & $8.28 e-02$ \\
\hline 3 & $(\theta, \phi)-(1 / 20)$ & $1.01 e-02$ & $8.76 e-03$ & $8.55 e-03$ \\
\hline 4 & $(0, \varphi)-(1 / 2,0)$ & $7.67 e-03$ & $3.55 e-05$ & $1.38 e-06$ \\
\hline 5 & & $7.67 e-03$ & $3.55 e-05$ & $1.60 e-09$ \\
\hline 6 & & $7.67 e-03$ & $3.55 e-05$ & $1.45 e-09$ \\
\hline 1 & & $3.81 e-01$ & $4.10 e-01$ & $4.09 e-01$ \\
\hline 2 & & $7.41 e-02$ & $8.39 e-02$ & $8.34 e-02$ \\
\hline 3 & Chebvshey polvnomials of second Kind $(\theta \alpha)=(1 / 21 / 2)$ & $1.12 e-02$ & $8.73 e-03$ & $8.65 e-03$ \\
\hline 4 & & $7.85 e-03$ & $1.29 e-05$ & $1.41 e-06$ \\
\hline 5 & & $7.85 e-03$ & $1.29 e-05$ & $2.38 e-09$ \\
\hline 6 & & $7.85 e-03$ & $1.29 e-05$ & $2.23 e-09$ \\
\hline 1 & & $3.78 e-01$ & $4.10 e-01$ & $4.09 e-01$ \\
\hline 2 & & $7.33 e-02$ & $8.38 e-02$ & $8.34 e-02$ \\
\hline 3 & Chebychey nolynomials of firct kind $(\theta-\phi)-(-1 / 2-1 / 2)$ & $1.25 e-02$ & $8.73 e-03$ & $8.65 e-03$ \\
\hline 4 & 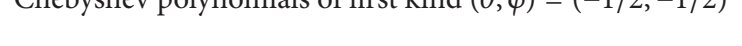 & $9.28 e-03$ & $1.58 e-05$ & $1.41 e-06$ \\
\hline 5 & & $9.28 e-03$ & $1.58 e-05$ & $3.63 e-10$ \\
\hline 6 & & $9.28 e-03$ & $1.58 e-05$ & $3.40 e-10$ \\
\hline
\end{tabular}

By discretization using J-GL-C methods we obtain

$$
\begin{aligned}
\mathbf{F}(\mathbf{u})= & \left(\mathbf{S}_{t t} \otimes \mathbf{I}_{x} \otimes \mathbf{I}_{y}\right) \mathbf{u} \\
& -c^{2}\left(\mathbf{I}_{t} \otimes \mathbf{S}_{x x} \otimes \mathbf{I}_{y}+\mathbf{I}_{t} \otimes \mathbf{I}_{x} \otimes \mathbf{S}_{y y}\right) \mathbf{u} \\
& +f(\mathbf{u})-\mathbf{p}=\mathbf{0}, \\
\mathbf{F}^{\prime}(\mathbf{u})= & \mathbf{S}_{t t} \otimes \mathbf{I}_{x} \otimes \mathbf{I}_{y} \\
& -c^{2}\left(\mathbf{I}_{t} \otimes \mathbf{S}_{x x} \otimes \mathbf{I}_{y}+\mathbf{I}_{t} \otimes \mathbf{I}_{x} \otimes \mathbf{S}_{y y}\right) \\
& +\operatorname{diag}\left(f^{\prime}(\mathbf{u})\right),
\end{aligned}
$$

where $\mathbf{u}=\left[u_{(1,1,1)}, u_{(1,1,2)}, \ldots, u_{\left(1,1, n_{t}\right)}, \ldots, u_{\left(n_{x}, n_{y}, 1\right)}, u_{\left(n_{x}, n_{y}, 2\right)}\right.$, $\left.\ldots, u_{\left(n_{x}, n_{y}, n_{z}\right)}\right]^{T}, \mathbf{S}_{t t}=\left(\left(2 / t_{f}\right) \mathbf{Q}_{t}\right)^{2}, \mathbf{S}_{x x}=\left(\left(2 /\left(b_{x}-a_{x}\right)\right) \mathbf{Q}_{x}\right)^{2}$, and $\mathbf{S}_{y y}=\left(\left(2 /\left(b_{y}-a_{y}\right)\right) \mathbf{Q}_{y}\right)^{2}$. The solution of (39) with $c=1$, $f(u)=u^{3}$ and appropriate initial and boundary conditions is $e^{-t} \sin (x+y)$. We took a domain $(x, y, t) \in[-1,1] \times[-1,1] \times$ $[0,2]$ and solved the system of nonlinear equations using a single application of NMIM with initial guess $\mathbf{u}=\mathbf{0}$ with the initial and boundary conditions introduced. Table 14 gives the norm of the error as a function of $m$ for several pairs of values

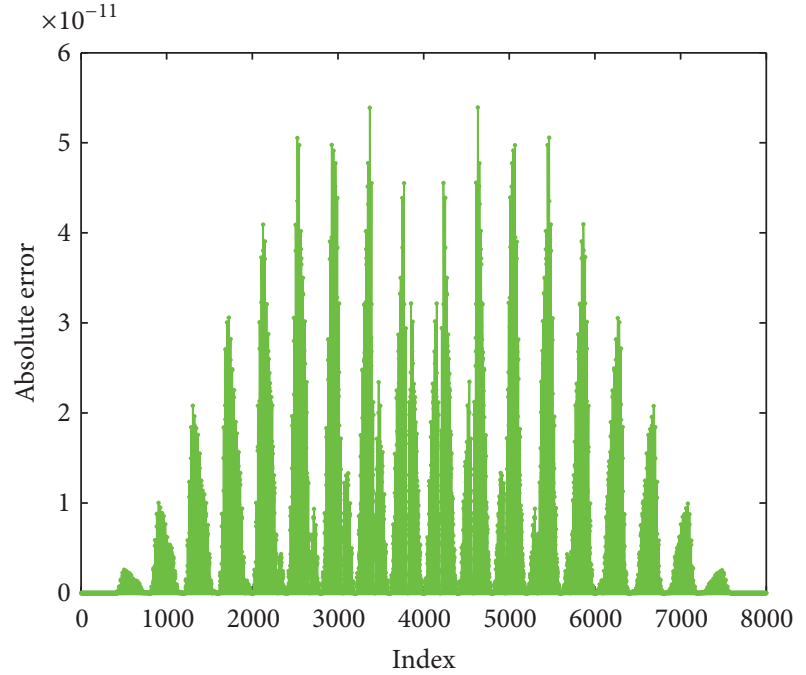

FIGURE 9: Error components for the nonlinear 2D wave equation.

for $\theta$ and $\phi$ and several grids. The collocation method with $20 \times 20 \times 20$ which worked best was the Chebyshev collocation method of first kind. Figure 9 gives the error components for that collocation method and that grid. 
TABLE 14: Norm of the error for the 2D nonlinear wave equation as a function of $m$ for several pair of values for $\theta$ and $\phi$ and several grids.

\begin{tabular}{|c|c|c|c|c|}
\hline \multicolumn{5}{|c|}{$\mid \mathbf{u}-\mathbf{u}_{\text {analytical }} \|_{\infty}$} \\
\hline$m$ & & & Grid size & \\
\hline$m$ & & $5 \times 5 \times 20$ & $10 \times 10 \times 20$ & $20 \times 20 \times 20$ \\
\hline 1 & \multirow{4}{*}{ Legendre polynomials $(\theta, \phi)=(0,0)$} & $4.21 e-02$ & \multirow{4}{*}{ Divergent } & \multirow{4}{*}{ Divergent } \\
\hline 2 & & $3.85 e-03$ & & \\
\hline 3 & & $2.03 e-04$ & & \\
\hline 9 & & $1.09 e-04$ & & \\
\hline 1 & \multirow{5}{*}{$(\theta, \phi)=(1 / 2,0)$} & $4.45 e-02$ & $5.12 e-02$ & $5.14 e-02$ \\
\hline 2 & & $3.50 e-03$ & $3.74 e-03$ & $1.53 e-02$ \\
\hline 5 & & $7.34 e-04$ & $5.74 e-08$ & $2.16 e-04$ \\
\hline 7 & & $7.34 e-04$ & $1.40 e-09$ & $3.76 e-06$ \\
\hline 9 & & $7.34 e-04$ & $1.40 e-09$ & $6.56 e-08$ \\
\hline 1 & \multirow{5}{*}{ Chebyshev polynomials of second kind $(\theta, \phi)=(1 / 2,1 / 2)$} & $4.40 e-02$ & $5.13 e-02$ & \multirow{5}{*}{ Divergent } \\
\hline 2 & & $3.43 e-03$ & $3.81 e-03$ & \\
\hline 3 & & $5.98 e-04$ & $1.62 e-04$ & \\
\hline 4 & & $4.39 e-04$ & $8.01 e-09$ & \\
\hline 5 & & $4.39 e-04$ & $7.64 e-10$ & \\
\hline 1 & \multirow{6}{*}{ Chebyshev polynomials of first kind $(\theta, \phi)=(-1 / 2,-1 / 2)$} & $3.88 e-02$ & $5.02 e-02$ & $5.13 e-02$ \\
\hline 2 & & $3.91 e-03$ & $3.85 e-03$ & $3.80 e-03$ \\
\hline 3 & & $5.94 e-04$ & $1.54 e-04$ & $1.79 e-03$ \\
\hline 4 & & $6.60 e-04$ & $4.54 e-09$ & $4.28 e-05$ \\
\hline 5 & & $6.60 e-04$ & $6.78 e-10$ & $7.94 e-07$ \\
\hline 9 & & $6.60 e-04$ & $6.78 e-10$ & $5.39 e-11$ \\
\hline
\end{tabular}

5.2.7. 3D Nonlinear Wave Equation. The 3D nonlinear wave equation is

$$
\begin{aligned}
& u_{t t}(x, y, z, t)-c^{2}\left(u_{x x}(x, y, z, t)+u_{y y}(x, y, z, t)\right. \\
& \left.+u_{z z}(x, y, z, t)\right)+f(u)=p(x, y, z), \\
& (x, y, z, t) \in D_{x} \times D_{y} \times D_{z} \times D_{t},
\end{aligned}
$$

$$
\begin{aligned}
& D_{z}=\left[a_{z}, b_{z}\right], \\
& D_{t}=\left[0, t_{f}\right],
\end{aligned}
$$

with the initial and boundary conditions

$$
\begin{aligned}
& u\left(a_{x}, y, z, t\right)=\varrho_{11}(y, z, t), \\
& u\left(b_{x}, y, z, t\right)=\varrho_{12}(y, z, t),
\end{aligned}
$$

$$
\begin{aligned}
& u\left(x, a_{y}, z, t\right)=\varrho_{21}(x, z, t), \\
& u\left(x, b_{y}, z, t\right)=\varrho_{22}(x, z, t), \\
& u\left(x, y, a_{z}, t\right)=\varrho_{31}(x, y, t), \\
& u\left(x, y, b_{z}, t\right)=\varrho_{32}(x, y, t), \\
& u(x, y, z, 0)=\zeta(x, y, z) .
\end{aligned}
$$

By discretization using J-GL-C methods we obtain

$$
\begin{aligned}
& \mathbf{F}(\mathbf{u})=\left(\mathbf{S}_{t t} \otimes \mathbf{I}_{x} \otimes \mathbf{I}_{y} \otimes \mathbf{I}_{z}\right) \mathbf{u}-c^{2}\left(\mathbf{I}_{t} \otimes \mathbf{S}_{x x} \otimes \mathbf{I}_{y} \otimes \mathbf{I}_{z}\right. \\
& \left.+\mathbf{I}_{t} \otimes \mathbf{I}_{x} \otimes \mathbf{S}_{y y} \otimes \mathbf{I}_{z}+\mathbf{I}_{t} \otimes \mathbf{I}_{x} \otimes \mathbf{I}_{y} \otimes \mathbf{S}_{z z}\right) \mathbf{u}+f(\mathbf{u}) \\
& \quad-\mathbf{p}=\mathbf{0}, \\
& \mathbf{F}^{\prime}(\mathbf{u})=\left(\mathbf{S}_{t t} \otimes \mathbf{I}_{x} \otimes \mathbf{I}_{y} \otimes \mathbf{I}_{z}\right)-c^{2}\left(\mathbf{I}_{t} \otimes \mathbf{S}_{x x} \otimes \mathbf{I}_{y} \otimes \mathbf{I}_{z}\right. \\
& \left.\quad+\mathbf{I}_{t} \otimes \mathbf{I}_{x} \otimes \mathbf{S}_{y y} \otimes \mathbf{I}_{z}+\mathbf{I}_{t} \otimes \mathbf{I}_{x} \otimes \mathbf{I}_{y} \otimes \mathbf{S}_{z z}\right) \\
& \quad+\operatorname{diag}\left(f^{\prime}(\mathbf{u})\right)
\end{aligned}
$$

where $\mathbf{u}=\left[u_{(1,1,1,1)}, u_{(1,1,1,2)}, \ldots, u_{\left(1,1,1, n_{t}\right)}, \ldots, u_{\left(n_{x}, n_{y}, n_{z}, 1\right)}\right.$, $\left.u_{\left(n_{x}, n_{y}, n_{z}, 2\right)}, \ldots, u_{\left(n_{x}, n_{y}, n_{z}, n_{t}\right)}\right]^{T}, \mathbf{S}_{t t}=\left(\left(2 / t_{f}\right) \mathbf{Q}_{t}\right)^{2}, \mathbf{S}_{x x}=$ $\left(\left(2 /\left(b_{x}-a_{x}\right)\right) \mathbf{Q}_{x}\right)^{2}, \mathbf{S}_{y y}=\left(\left(2 /\left(b_{y}-a_{y}\right)\right) \mathbf{Q}_{y}\right)^{2}$, and 
TABLE 15: Norm of the error for the 3D nonlinear wave equation as a function of $m$ for several values for $\theta$ and $\phi$ and grids.

\begin{tabular}{|c|c|c|c|c|}
\hline \multirow{2}{*}{\multicolumn{5}{|c|}{$\mathbf{u}-\mathbf{u}_{\text {analytical }} \|_{\infty}$}} \\
\hline$m$ & & & & \\
\hline$m$ & & $5 \times 5 \times 5 \times 12$ & $6 \times 6 \times 6 \times 12$ & $7 \times 7 \times 7 \times 12$ \\
\hline 1 & \multirow{5}{*}{ Legendre polynomials $(\theta, \phi)=(0,0)$} & $9.95 e-01$ & $1.00 e+00$ & $1.14 e+00$ \\
\hline 3 & & $1.14 e-02$ & $8.53 e-03$ & $1.09 e-02$ \\
\hline 5 & & $1.25 e-01$ & $1.12 e-01$ & $1.73 e-01$ \\
\hline 7 & & $5.53 e-06$ & $4.12 e-07$ & $1.73 e-06$ \\
\hline 8 & & $5.54 e-06$ & $7.54 e-08$ & $4.24 e-09$ \\
\hline 1 & \multirow{5}{*}{$(\theta, \phi)=(1 / 2,0)$} & $1.00 e+00$ & $1.00 e+00$ & \multirow{5}{*}{ divergent } \\
\hline 2 & & $1.00 e-01$ & $8.68 e-02$ & \\
\hline 4 & & $1.31 e+00$ & $1.29 e+00$ & \\
\hline 7 & & $2.24 e-05$ & $1.99 e-06$ & \\
\hline 8 & & $2.24 e-05$ & $2.11 e-06$ & \\
\hline 1 & \multirow{5}{*}{ Chebyshev polynomials of second kind $(\theta, \phi)=(1 / 2,1 / 2)$} & $9.98 e-01$ & $9.99 e-01$ & $1.32 e+00$ \\
\hline 2 & & $1.02 e-01$ & $8.06 e-02$ & $1.08 e-01$ \\
\hline 5 & & $1.22 e-01$ & $1.18 e-01$ & $2.46 e-01$ \\
\hline 7 & & $1.81 e-05$ & $1.84 e-06$ & $3.72 e-05$ \\
\hline 8 & & $1.81 e-05$ & $1.76 e-06$ & $4.87 e-08$ \\
\hline 1 & \multirow{5}{*}{ Chebyshev polynomials of first kind $(\theta, \phi)=(-1 / 2,-1 / 2)$} & $9.95 e-01$ & \multirow{5}{*}{ divergent } & $1.09 e+00$ \\
\hline 2 & & $9.98 e-02$ & & $1.04 e-01$ \\
\hline 5 & & $1.31 e-01$ & & $1.51 e-01$ \\
\hline 7 & & $1.92 e-05$ & & $4.37 e-07$ \\
\hline 8 & & $1.92 e-05$ & & $3.27 e-08$ \\
\hline
\end{tabular}

$\mathbf{S}_{z z}=\left(\left(2 /\left(b_{z}-a_{z}\right)\right) \mathbf{Q}_{z}\right)^{2}$. The solution of (43) with $c=0.8$, $f(u)=u^{2}$ and appropriate initial and boundary conditions is $e^{-t} \sin (x+y+z)$. We took a domain $(x, y, z, t) \in[0,1] \times[0,1] \times$ $[0,1] \times[0,1]$ and solved the system of nonlinear equations using a single application of NMIM with initial guess $\mathbf{u}=\mathbf{0}$ and with initial and boundary conditions introduced. Table 15 gives the norm of the error as a function of $m$ for several pairs of values for $\theta, \phi$ and several grids. The collocation method which worked best for the grid $7 \times 7 \times 7 \times 12$ was the Legendre collocation method. Figure 10 gives the error components for that collocation method and that grid.

5.2.8. 3D Nonlinear Poisson Equation. The 3D nonlinear Poisson equation is

$$
\begin{gathered}
u_{x x}(x, y, z)+u_{y y}(x, y, z)+u_{z z}(x, y, z)+f(u) \\
=p(x, y, z), \quad(x, y, z) \in D_{x} \times D_{y} \times D_{z},
\end{gathered}
$$

where

$$
\begin{aligned}
& D_{x}=\left[a_{x}, b_{x}\right], \\
& D_{y}=\left[a_{y}, b_{y}\right], \\
& D_{z}=\left[a_{z}, b_{z}\right],
\end{aligned}
$$

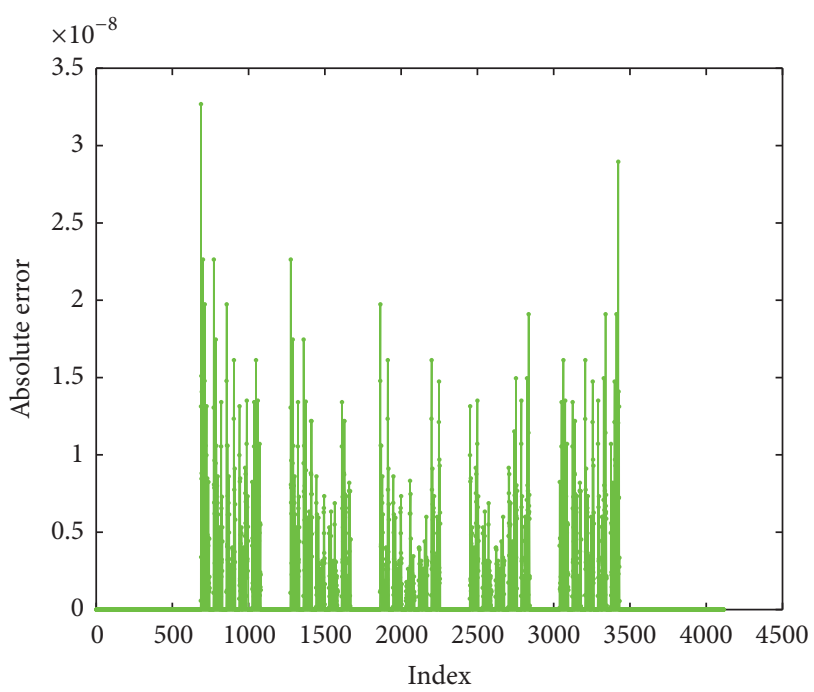

FIGURE 10: Error components for the nonlinear 3D wave equation.

with the initial and boundary conditions

$$
\begin{aligned}
& u\left(a_{x}, y, z\right)=\varrho_{11}(y, z), \\
& u\left(b_{x}, y, z\right)=\varrho_{12}(y, z), \\
& u\left(x, a_{y}, z\right)=\varrho_{21}(x, z),
\end{aligned}
$$




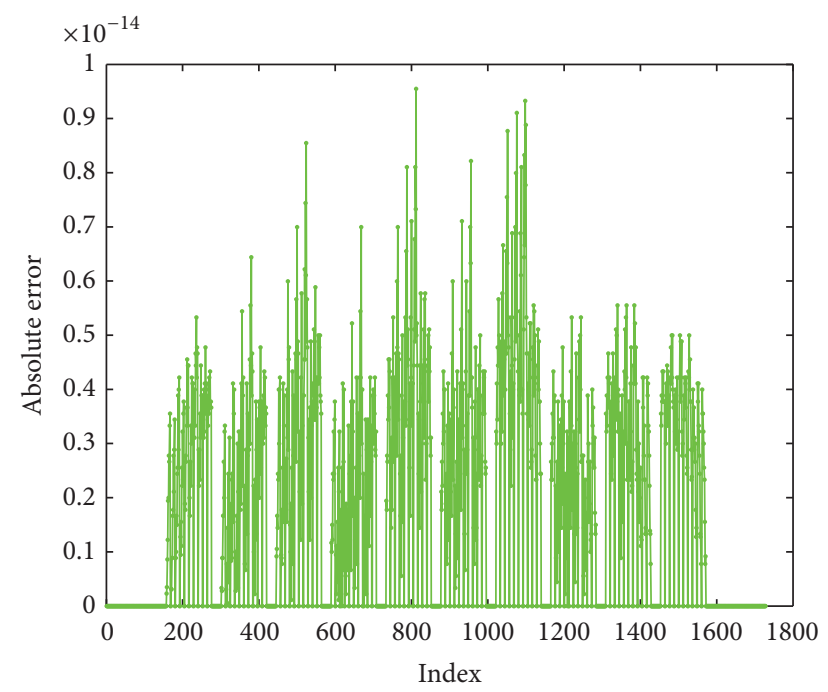

FIGURE 11: Error components for the nonlinear 3D Poisson equation.

$$
\begin{aligned}
& u\left(x, b_{y}, z\right)=\varrho_{22}(x, z), \\
& u\left(x, y, a_{z}\right)=\varrho_{31}(x, y), \\
& u\left(x, y, b_{z}\right)=\varrho_{32}(x, y) .
\end{aligned}
$$

By discretization using J-GL-C methods we obtain

$\mathbf{F}(\mathbf{u})$

$$
\begin{aligned}
= & \left(\mathbf{S}_{x x} \otimes \mathbf{I}_{y} \otimes \mathbf{I}_{z}+\mathbf{I}_{x} \otimes \mathbf{S}_{y y} \otimes \mathbf{I}_{z}+\mathbf{I}_{x} \otimes \mathbf{I}_{y} \otimes \mathbf{S}_{z z}\right) \mathbf{u} \\
& +f(\mathbf{u})-\mathbf{p}=\mathbf{0},
\end{aligned}
$$

$\mathbf{F}^{\prime}(\mathbf{u})$

$$
\begin{aligned}
= & \left(\mathbf{S}_{x x} \otimes \mathbf{I}_{y} \otimes \mathbf{I}_{z}+\mathbf{I}_{x} \otimes \mathbf{S}_{y y} \otimes \mathbf{I}_{z}+\mathbf{I}_{x} \otimes \mathbf{I}_{y} \otimes \mathbf{S}_{z z}\right) \\
& +\operatorname{diag}\left(f^{\prime}(\mathbf{u})\right)
\end{aligned}
$$

where $\mathbf{u}=\left[u_{(1,1,1)}, u_{(1,1,2)}, \ldots, u_{\left(1,1, n_{z}\right)}, \ldots, u_{\left(n_{x}, n_{y}, 1\right)}, u_{\left(n_{x}, n_{y}, 2\right)}\right.$, $\left.\ldots, u_{\left(n_{x}, n_{y}, n_{z}\right)}\right]^{T}, \mathbf{S}_{x x}=\left(\left(2 /\left(b_{x}-a_{x}\right)\right) \mathbf{Q}_{x}\right)^{2}, \mathbf{S}_{y y}=\left(\left(2 /\left(b_{y}-\right.\right.\right.$ $\left.\left.\left.a_{y}\right)\right) \mathbf{Q}_{y}\right)^{2}$, and $\mathbf{S}_{z z}=\left(\left(2 /\left(b_{z}-a_{z}\right)\right) \mathbf{Q}_{z}\right)^{2}$. The solution of (47) with $f(u)=u^{4}$ with appropriate initial and boundary conditions is $\sin (x+y+z)$. We took a domain $(x, y, z) \in$ $[0,1] \times[0,1] \times[0,1]$ and solved the system of nonlinear equations using a single application of NMIM with initial guess $\mathbf{u}=\mathbf{0}$ and with initial and boundary conditions introduced. Table 16 gives the norm of the error as a function of $m$ for several pairs of values for $\theta$ and $\phi$ and several grids. The Legendre collocation method offers best accuracy over different grids. Figure 11 gives the error components for that collocation method and that grid.

5.2.9. The Nonlinear Complex Generalized Zakharov System. The nonlinear complex Zakharov system has importance in plasma physics [3]. The system includes two coupled nonlinear PDEs which can be written as

$$
\begin{aligned}
& i \partial_{t} \psi(x, t)+\delta_{1} \partial_{t t} \psi(x, t)-\delta_{2} \psi(x, t) w(x, t) \\
&+\delta_{3}|\psi(x, t)|^{2} \psi(x, t)= 0, \\
& \partial_{t t} w(x, t)-c_{s}^{2} \partial_{x x} w(x, t)-\delta_{4} \partial_{x x}|\psi(x, t)|^{2}=0, \\
& \quad(x, t) \in\left(a_{x}, b_{x}\right) \times\left(a_{t}, b_{t}\right),
\end{aligned}
$$

subject to the initial and boundary conditions

$$
\begin{aligned}
& \psi\left(a_{x}, t\right)=\psi_{1}(t), \\
& \psi\left(b_{x}, t\right)=\psi_{2}(t), \\
& \psi(x, 0)=\psi_{0}(x), \\
& w\left(a_{x}, t\right)=w_{1}(t), \\
& w\left(b_{x}, t\right)=w_{2}(x), \\
& w(x, 0)=w_{3}(x),
\end{aligned}
$$

$$
(x, t) \in\left[a_{x}, b_{x}\right] \times\left[a_{t}, b_{t}\right] .
$$

Several numerical methods have been proposed recently for approximating the solution of (51) and (52) such as the homotopy method [24], the finite difference method $[25,26]$, and the variational iteration method [27]. Also, Bao et al. [28] suggested some high-accurate numerical methods for solving numerically (51) and (52). Bao and Sun [29] applied a new technique based on time-splitting discretization for approximating the solution of a variant of (51) and (52).

One can split (51) using the real and imaginary parts of $\psi(x, t), u(x, t)$, and $v(x, t)$, as

$$
\begin{aligned}
& \partial_{t} u(x, t)+\delta_{1} \partial_{x x} v(x, t)-\delta_{2} v(x, t) w(x, t) \\
& \quad+\delta_{3}\left(u^{2}(x, t)+v^{2}(x, t)\right) v(x, t)=0 \\
& -\partial_{t} v(x, t)+\delta_{1} \partial_{x x} u(x, t)-\delta_{2} u(x, t) w(x, t) \\
& \quad+\delta_{3}\left(u^{2}(x, t)+v^{2}(x, t)\right) u(x, t)=0 \\
& \partial_{t t} w(x, t)-c_{s}^{2} \partial_{x x} w(x, t)-2 \delta_{4}\left(u(x, t) \partial_{x x} u(x, t)\right. \\
& \left.\quad+\left(\partial_{x} u(x, t)\right)^{2}+v(x, t) \partial_{x x} v(x, t)+\left(\partial_{x} v(x, t)\right)^{2}\right) \\
& \quad=0
\end{aligned}
$$

with the initial and boundary conditions

$$
\begin{aligned}
& u\left(a_{x}, t\right)=\alpha_{1}(t), \\
& u\left(b_{x}, t\right)=\alpha_{2}(t), \\
& v\left(a_{x}, t\right)=\alpha_{3}(t), \\
& v\left(b_{x}, t\right)=\alpha_{4}(t), \\
& w\left(a_{x}, t\right)=\alpha_{5}(t), \\
& w\left(b_{x}, t\right)=\alpha_{6}(t),
\end{aligned}
$$


TABLE 16: Norm of the error for the 3D Nonlinear Poisson equation as a function of $m$ for several values for $\theta$ and $\phi$ and grids.

\begin{tabular}{|c|c|c|c|c|}
\hline & $\| \mathbf{u}-\mathbf{u}_{\text {anal }}$ & & & \\
\hline$m$ & & & Grid size & \\
\hline$m$ & & $8 \times 8 \times 8$ & $10 \times 10 \times 10$ & $12 \times 12 \times 12$ \\
\hline 1 & & $4.29 e-02$ & $4.44 e-02$ & $4.52 e-02$ \\
\hline 4 & Jeoendre polynomials $(\theta-\phi)-(0-0)$ & $5.54 e-08$ & $6.03 e-08$ & $6.30 e-08$ \\
\hline 5 & Legentire porynuminals $(0, \psi)-(0,0)$ & $2.85 e-11$ & $8.77 e-12$ & $9.27 e-12$ \\
\hline 6 & & $2.95 e-11$ & $1.51 e-14$ & $6.44 e-15$ \\
\hline 1 & & $4.37 e-02$ & $4.51 e-02$ & $4.57 e-02$ \\
\hline 3 & $(\theta-\phi)=(1 / 20)$ & $6.80 e-04$ & $7.09 e-04$ & $7.24 e-04$ \\
\hline 5 & $(0,4)-(1 / 2,0)$ & $1.33 e-09$ & $9.88 e-12$ & $9.75 e-12$ \\
\hline 6 & & $1.33 e-09$ & $9.39 e-13$ & $7.66 e-15$ \\
\hline 1 & & $4.33 e-02$ & $4.46 e-02$ & $4.53 e-02$ \\
\hline 2 & Chebychey nolynominls of cecond kind $(\theta-\phi)-(1 / 21 / 2)$ & $5.39 e-03$ & $5.63 e-03$ & $5.76 e-03$ \\
\hline 4 & 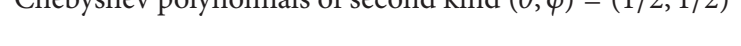 & $5.58 e-08$ & $6.10 e-08$ & $6.35 e-08$ \\
\hline 6 & & $9.59 e-10$ & $6.78 e-13$ & $1.88 e-14$ \\
\hline 1 & & $4.23 e-02$ & $4.41 e-02$ & $4.50 e-02$ \\
\hline 3 & Chebyshey polynomials of first kind $(\theta, \phi)=(-1 / 2,-1 / 2)$ & $6.33 e-04$ & $6.78 e-04$ & $7.01 e-04$ \\
\hline 5 & & $5.63 e-10$ & $8.53 e-12$ & $9.17 e-12$ \\
\hline 6 & & $5.63 e-10$ & $3.26 e-13$ & $8.55 e-15$ \\
\hline
\end{tabular}

$$
\begin{gathered}
u\left(x, a_{t}\right)=\beta_{1}(x), \\
v\left(x, a_{t}\right)=\beta_{2}(x), \\
w\left(x, a_{t}\right)=\beta_{3}(x), \\
w_{t}\left(x, a_{t}\right)=\beta_{4}(x),
\end{gathered}
$$$$
(x, t) \in\left[a_{x}, b_{x}\right] \times\left[a_{t}, b_{t}\right] .
$$

The matrix form of the nonlinear system (53) is

$$
\begin{gathered}
{\left[\begin{array}{ccc}
\partial_{t} & \delta_{1} \partial_{x x} & 0 \\
\delta_{1} \partial_{x x} & -\partial_{t} & 0 \\
0 & 0 & \partial_{t t}-c_{s}^{2} \partial_{x x}
\end{array}\right]\left[\begin{array}{l}
u \\
v \\
w
\end{array}\right]+\left[\begin{array}{l}
q_{1}(u, v, w) \\
q_{2}(u, v, w) \\
q_{3}(u, v, w)
\end{array}\right]} \\
\quad=\left[\begin{array}{l}
0 \\
0 \\
0
\end{array}\right],
\end{gathered}
$$

where

$$
\begin{aligned}
q_{1} & =-\delta_{2} v(x, t) w(x, t)+\delta_{3}\left(u^{2}(x, t)+v^{2}(x, t)\right) \\
& \cdot v(x, t), \\
q_{2} & =-\delta_{2} u(x, t) w(x, t)+\delta_{3}\left(u^{2}(x, t)+v^{2}(x, t)\right) \\
& \cdot u(x, t), \\
q_{3} & =-2 \delta_{4}\left(u(x, t) \partial_{x x} u(x, t)+\left(\partial_{x} u(x, t)\right)^{2}\right. \\
& \left.+v(x, t) \partial_{x x} v \text { problem }(x, t)+\left(\partial_{x} v(x, t)\right)^{2}\right),
\end{aligned}
$$

where the constants $\delta_{1}, \delta_{2}, \delta_{3}, \delta_{4}$, and $c_{s}$ are given and the functions $\alpha_{i}(t), 1 \leq i \leq 6$ and $\beta_{j}(x), 1 \leq j \leq 4$ are known. We use J-GL-C methods for discretizing (55) subject to the initial and boundary conditions (54) to reduce (55) to a system of nonlinear algebraic equations.

5.2.10. Complex Generalized Zakharov Equation I. The first complex generalized Zakharov problem [3] is

$$
\begin{aligned}
i \partial_{t} \psi-\partial_{x x} \psi-\psi w & =0, \\
\partial_{t t} w-\partial_{x x} w+\partial_{x x}|\psi|^{2} & =0,
\end{aligned}
$$

with domain $[-1,1] \times[0,3.0]$. The nonlinear problem $(57)$ has the analytical solution

$$
\begin{aligned}
\psi(x, t) & =u(x, t)+i v(x, t) \\
& =\sqrt{3} e^{i(x+t)} \tanh \left(\frac{1}{\sqrt{2}}(x+2 t)\right), \\
w(x, t) & =1-\tanh ^{2}\left(\frac{1}{\sqrt{2}}(x+2 t)\right) .
\end{aligned}
$$

We define

$$
\begin{gathered}
E=\max \left\{\left\|\mathbf{U}-\mathbf{U}_{\text {analytical }}\right\|_{\infty},\left\|\mathbf{V}-\mathbf{V}_{\text {analytical }}\right\|_{\infty},\right. \\
\left.\left\|\mathbf{W}-\mathbf{W}_{\text {analytical }}\right\|_{\infty}\right\}
\end{gathered}
$$

to check the numerical accuracy in the computed solutions. Problem (57) is discretized by using Chebyshev collocation method of first kind. We took the initial guess $\mathbf{0}$ with initial and boundary conditions introduced and a grid $50 \times 20$. The achieved numerical accuracy is shown in Table 17. Our computed solutions are better than those in [3] because the maximum accuracy in [3] is of the order of $10^{-7}$. The solutions and error components are plotted in Figures 12, 13, and 14. 

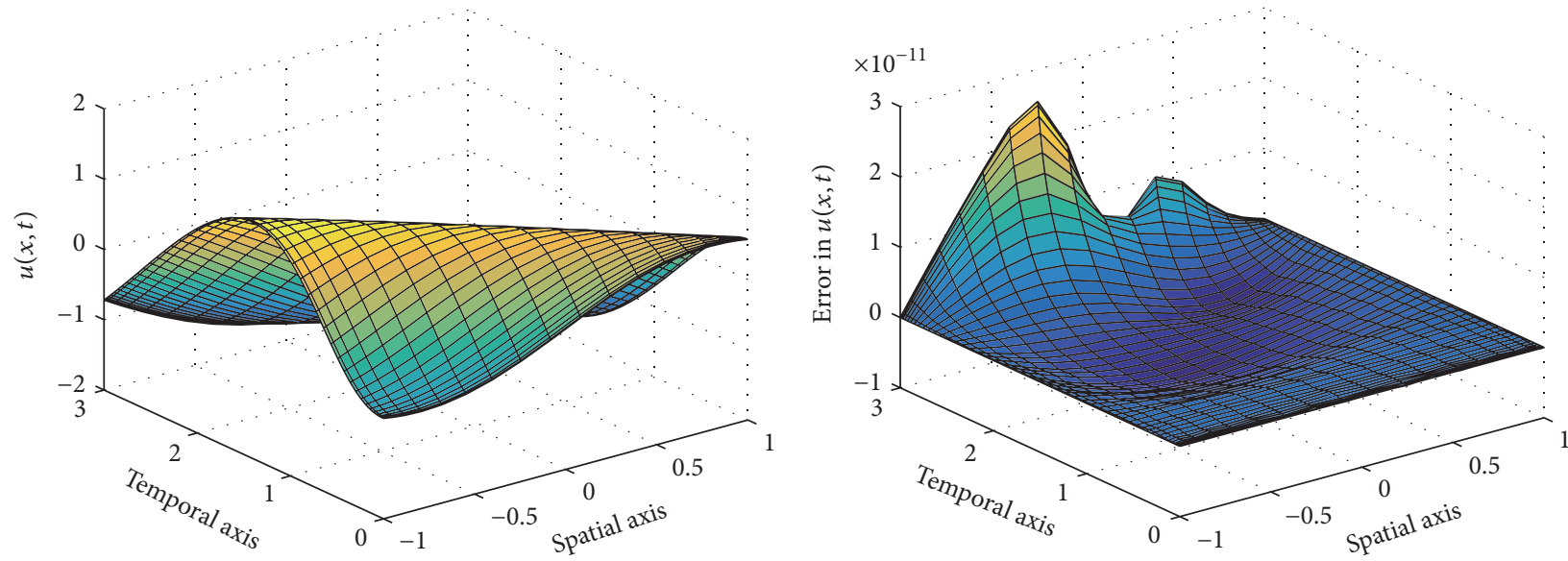

FIGURE 12: Solution and error components for complex generalized Zakharov equation (57).
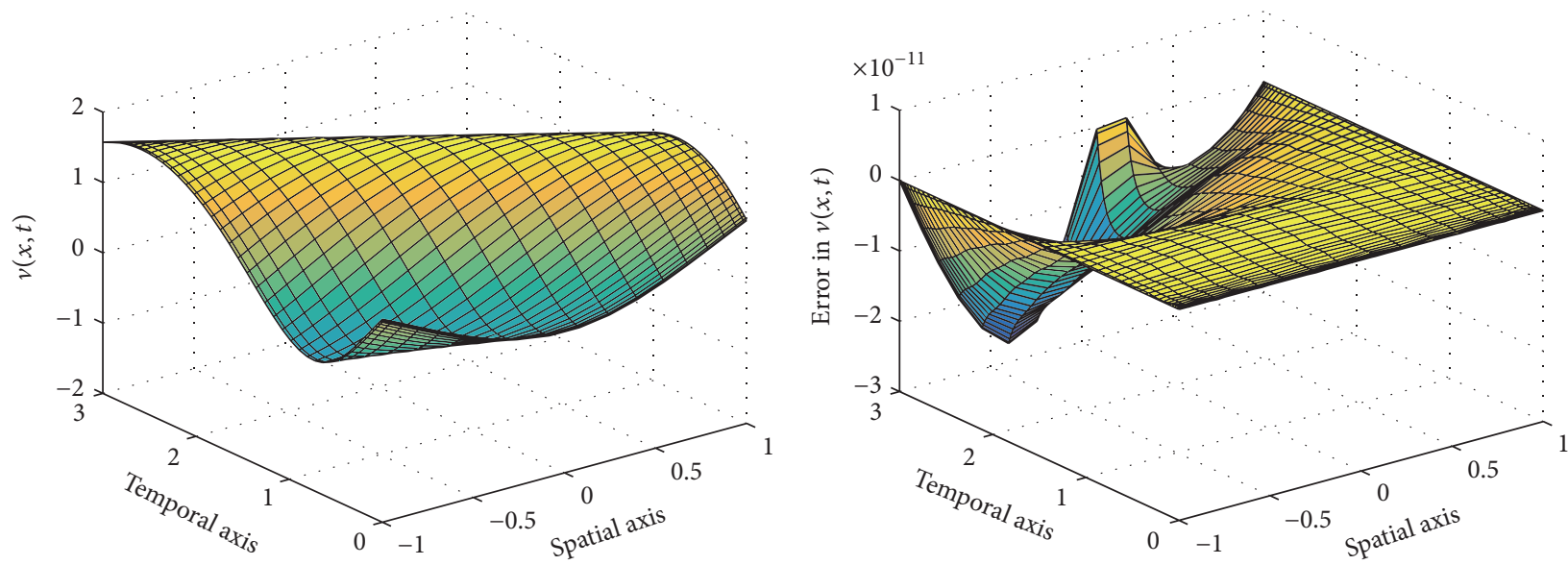

FIGURE 13: Solution and error components for complex generalized Zakharov equation (57).

5.2.11. Complex Generalized Zakharov Equation II. The second complex generalized Zakharov equation [3] is

$$
\begin{aligned}
i \partial_{t} \psi+\partial_{x x} \psi+2 \psi w-2|\psi|^{2} & =0, \\
\partial_{t t} w-\partial_{x x} w+\partial_{x x}|\psi|^{2} & =0
\end{aligned}
$$

with the domain $[-1,1] \times[0,1]$. That problem has the analytical solution

$$
\begin{aligned}
\psi(x, t) & =u(x, t)+i v(x, t) \\
& =\frac{\sqrt{3}}{2} e^{-i(x+3 t)} \tanh (x+2 t), \\
w(x, t) & =-\frac{1}{4} \tanh ^{2}(x+2 t) .
\end{aligned}
$$

Problem (60) is discretized by using Chebyshev collocation method of first kind. We took the initial guess $\mathbf{0}$ with initial and boundary conditions introduced and a grid $21 \times$ 28. The achieved numerical accuracy is shown in Table 18.
Our computed solutions are better than those in [3] because the ones in [3] achieved a maximum accuracy of the order of $10^{-6}$. The solutions and error components are plotted in Figures 15, 16, and 17.

\subsubsection{Murray Equation. Murray equation [2] is}

$$
\begin{aligned}
u_{t}=u_{x x}+\lambda_{1} u u_{x}+\lambda_{2} u-\lambda_{3} u^{2}, & \\
& (x, t) \in[-1,1] \times[0,1] .
\end{aligned}
$$

The initial and boundary condition for Murray equation can be computed from its analytical solution

$$
\begin{aligned}
& u(x, t)=\frac{\lambda_{2}}{2 \lambda_{3}}(1 \\
& \left.\quad+\tanh \left(\frac{\lambda_{2}}{8 \lambda_{3}^{2}}\left(2 \lambda_{1} \lambda_{3} x+\left(\lambda_{1}^{2}+4 \lambda_{3}^{2}\right) t\right)\right)\right) .
\end{aligned}
$$



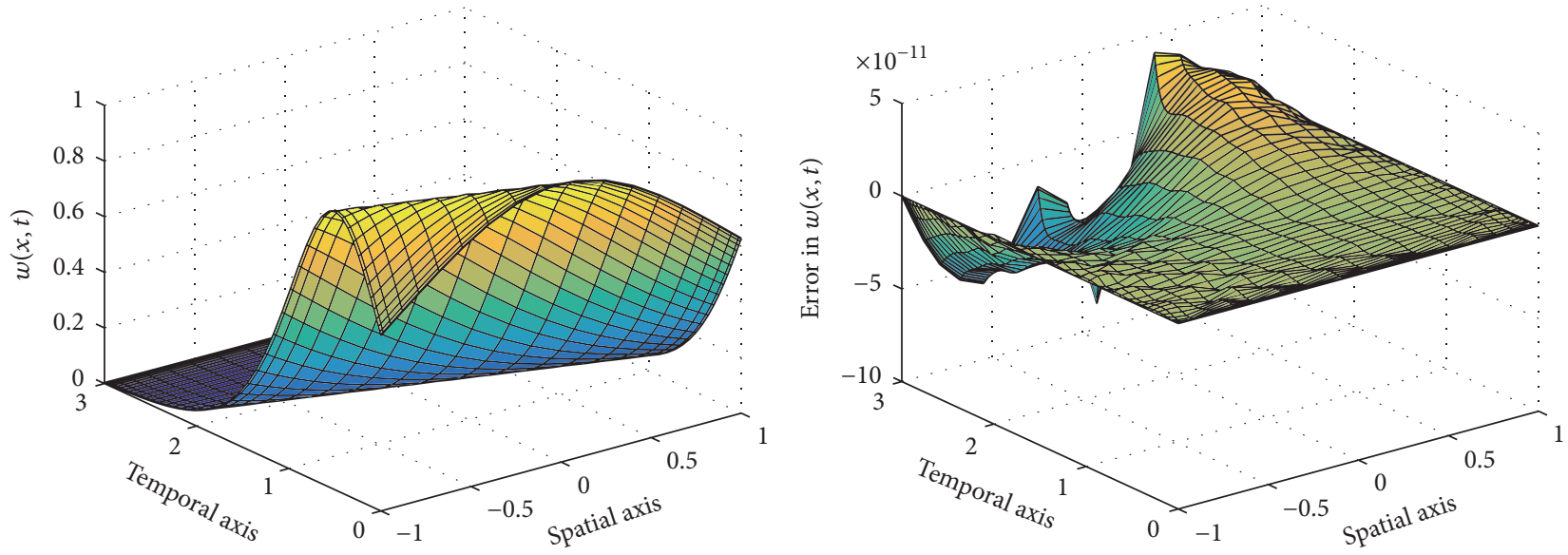

FIGURE 14: Solution and error components for complex generalized Zakharov equation (57).
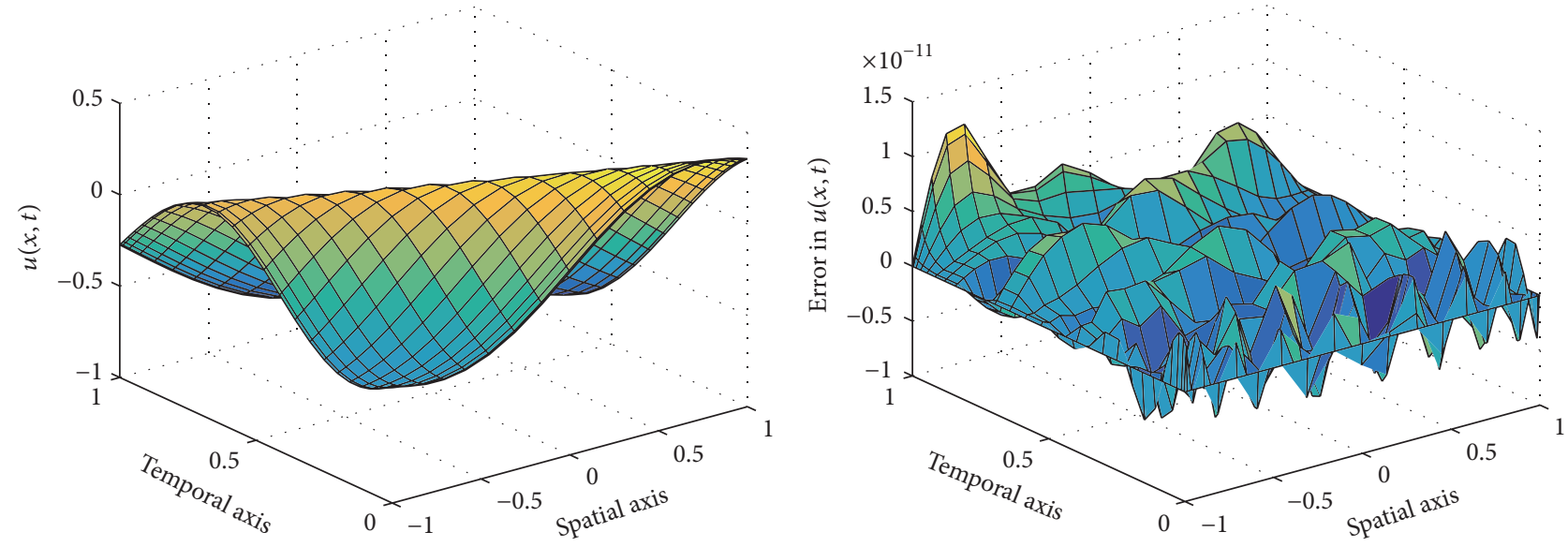

FIGURE 15: Solution and error components for complex generalized Zakharov equation (60).
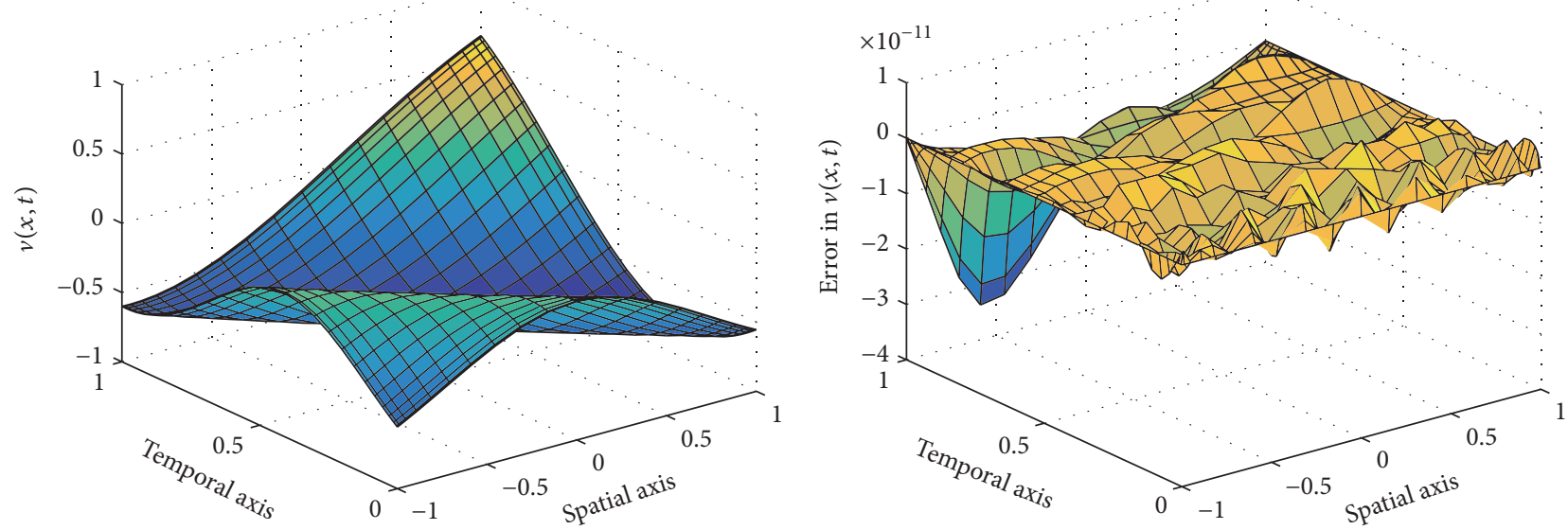

FIGURE 16: Solution and error components for complex generalized Zakharov equation (60). 

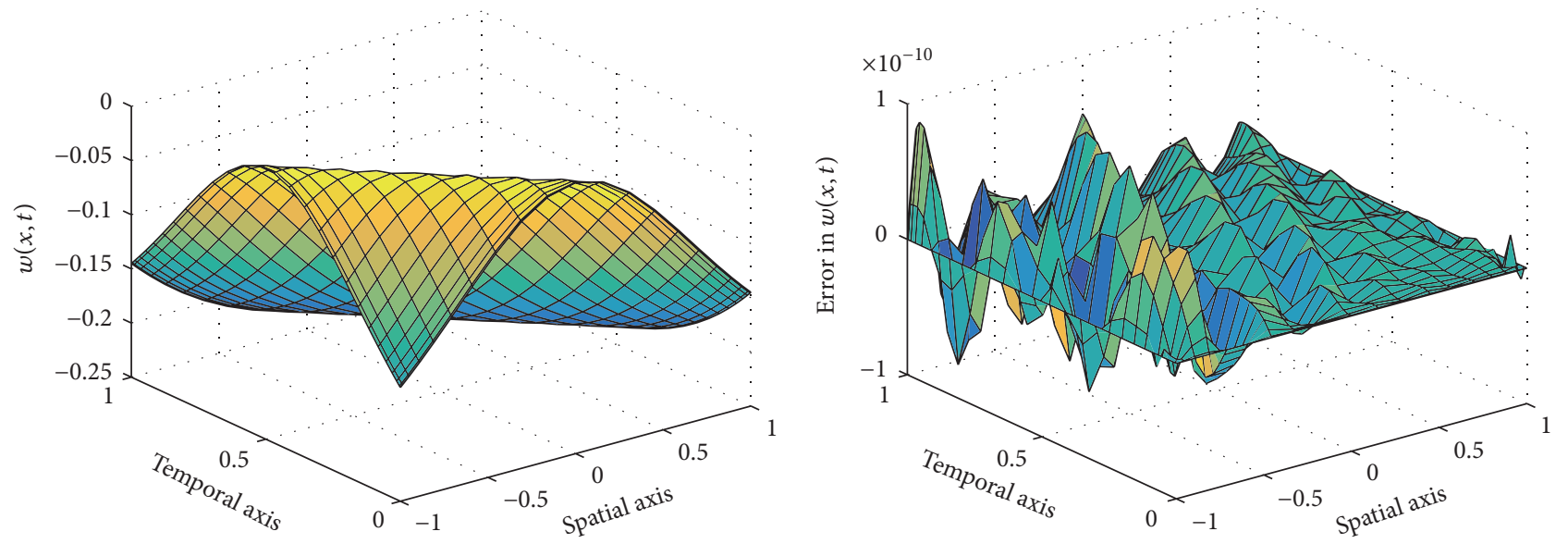

FIGURE 17: Solution and error components for complex generalized Zakharov equation (60).
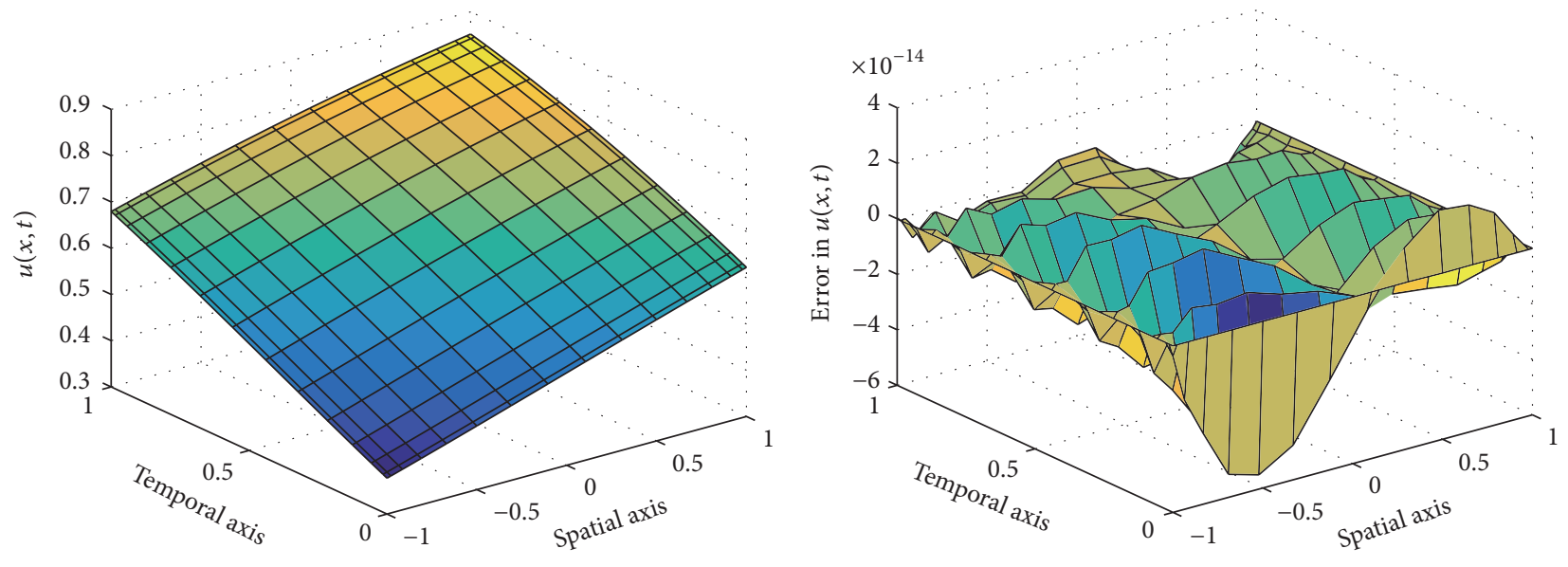

FIGURE 18: Solution and error components for Murray equation.

The Chebyshev collocation method of first kind is used to discretize Murray equation over the grid $16 \times 14$. The achieved numerical accuracy is shown in Table 19. The computed accuracy in [2] is of the order of $10^{-8}$. The solution and error components are shown in Figure 18.

\subsubsection{Nonlinear Reaction and Diffusion Equation. Nonlinear reaction and diffusion equation is}

$$
u_{t}=u_{x x}+u\left(1+u-u^{2}\right), \quad(x, t) \in[-1,1] \times[0,1] .
$$

The analytical solution is

$$
u(x, t)=\frac{1}{2}+\frac{\sqrt{5}}{2} \tanh \left(\frac{10}{4} x+\frac{5}{4} t\right) .
$$

The Chebyshev collocation method of first kind is used to discretize nonlinear reaction and diffusion equation over the grid $22 \times 14$. The initial guess is $\mathbf{0}$ with initial and boundary conditions introduced. The achieved numerical accuracy is shown in Table 20. The computed accuracy in [2] is of the order of $10^{-8}$. The solution and error components are shown in Figure 19.
5.2.14. Reaction-Diffusion Equation with Fischer-Kolmogorov Reaction Term and Density Dependent Diffusion. Reactiondiffusion equation with Fischer-Kolmogorov reaction term and density dependent diffusion is

$$
u_{t}=\left(u^{2}\right)_{x x}+u(1-u), \quad(x, t) \in[-1,1] \times[0,1] .
$$

The analytical solution is

$$
u(x, t)=\frac{2}{1+\tanh ((x+t) / 4)} .
$$

The Chebyshev collocation method of first kind is used to discretize reaction-diffusion equation with Fischer-Kolmogorov reaction term and density dependent diffusion over the grid $30 \times 30$. The initial guess is $\mathbf{0}$ with initial and boundary conditions introduced. The achieved numerical accuracy after 1 and 2 application of NMIM is shown in Table 21. The computed accuracy in [2] is only of the order of $10^{-9}$. The solution and error components are shown in Figure 20. 

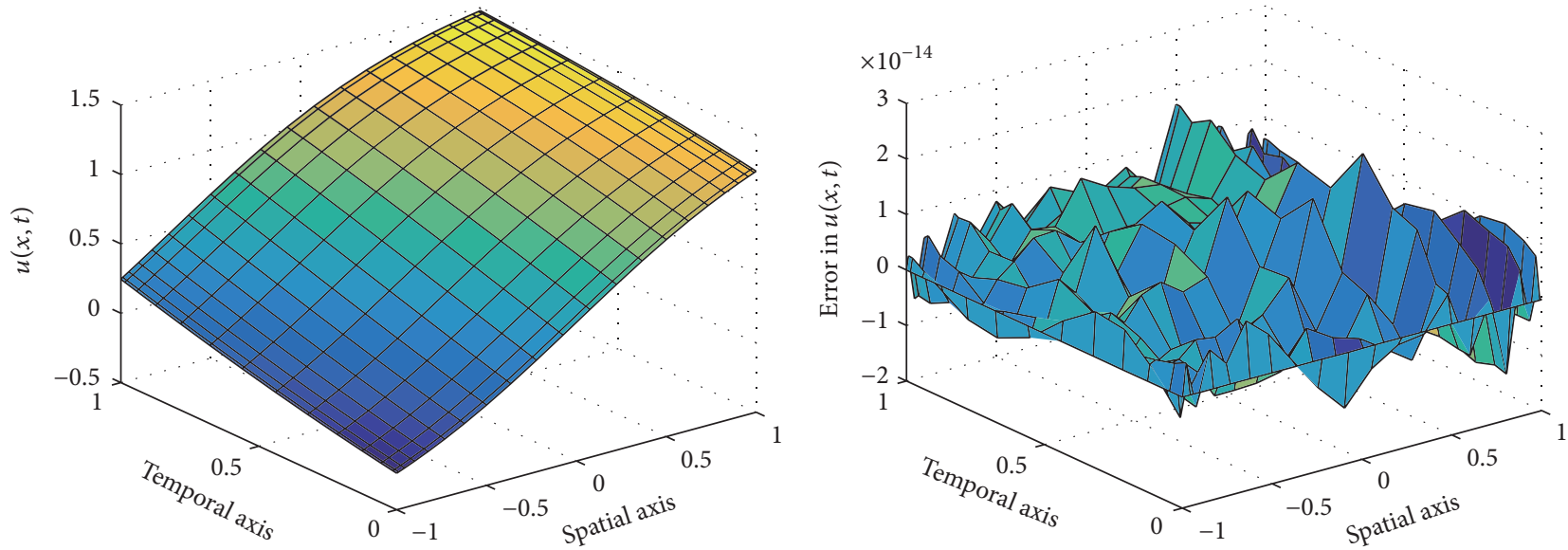

FIGURE 19: Solution and error components for nonlinear reaction and diffusion equation.
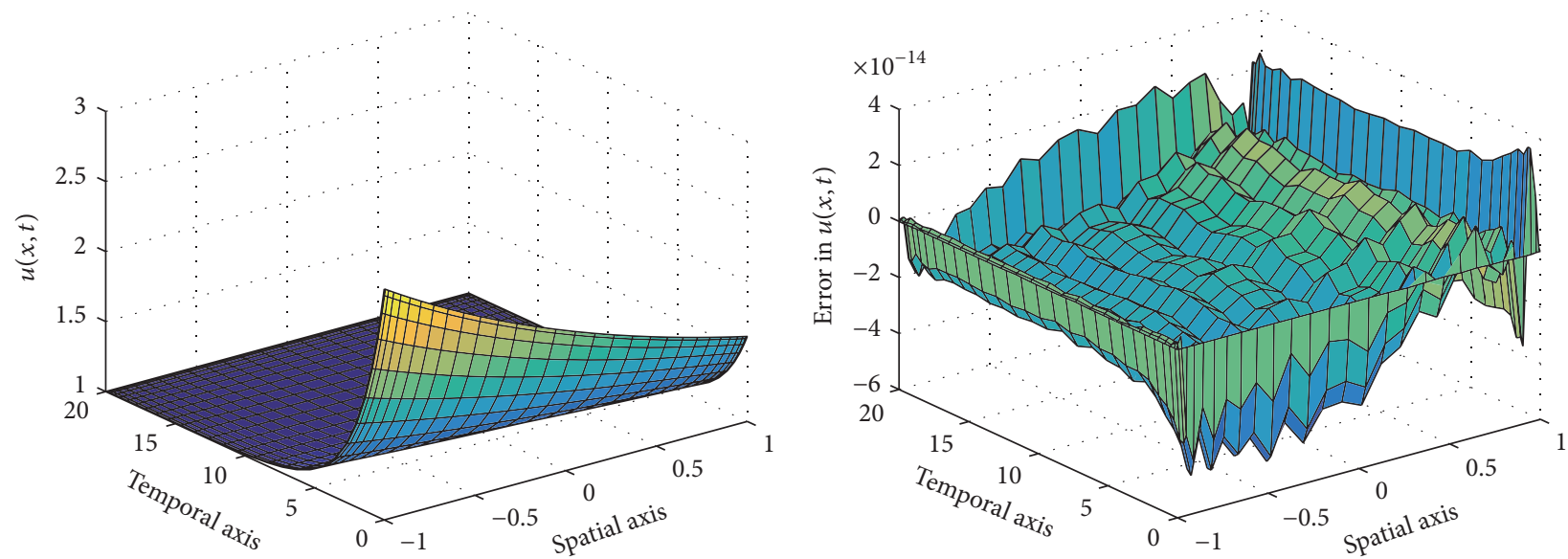

FIGURE 20: Solution and error components for reaction-diffusion equation with Fischer-Kolmogorov reaction term and density dependent diffusion.

5.2.15. Two-Dimensional Nonlinear Reaction-Diffusion Equation. The two-dimensional nonlinear reaction-diffusion equation is

$$
\begin{aligned}
u_{t}=\frac{1}{2} \nabla^{2} u+u^{2}(u-1), & \\
& (x, y, t) \in[-1,1] \times[-1,1] \times[0,20] .
\end{aligned}
$$

Its analytical solution is

$$
u(x, y, t)=\frac{1}{1+e^{(1 / \sqrt{2})(x+y-(1 / \sqrt{2}) t)}} .
$$

The Chebyshev collocation method of first kind is used to discretize the two-dimensional nonlinear reaction-diffusion equation over grid $30 \times 30$. The initial guess is $\mathbf{0}$ with initial and boundary conditions introduced. The achieved numerical accuracy is shown in Table 22. The computed accuracy in [2] is only of the order of $10^{-9}$. The error components are shown in Figure 21.

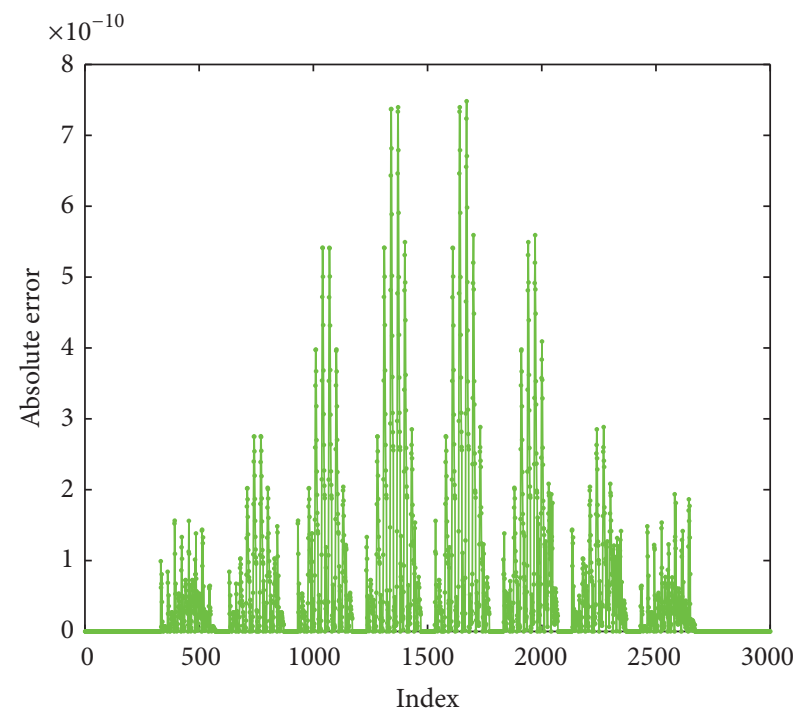

FIGURE 21: Error components for the two-dimensional nonlinear reaction-diffusion equation. 
TABLE 17: Maximum absolute error in the solution of the system of nonlinear equations for complex generalized Zakharov equation and varying $m$.

\begin{tabular}{|c|c|}
\hline$m$ & $E$ \\
\hline 1 & $8.56 e-1$ \\
\hline 2 & $2.72 e 0$ \\
\hline 3 & $4.16 e 0$ \\
\hline 4 & $8.92 e 0$ \\
\hline 5 & $1.12 e 1$ \\
\hline 6 & $3.40 e 1$ \\
\hline 7 & $2.04 e 1$ \\
\hline 8 & $2.83 e 1$ \\
\hline 9 & $4.05 e 0$ \\
\hline 10 & $2.34 e 0$ \\
\hline 11 & $7.77 e-1$ \\
\hline 12 & $3.25 e-1$ \\
\hline 13 & $1.14 e-1$ \\
\hline 14 & $6.20 e-2$ \\
\hline 15 & $2.79 e-2$ \\
\hline 16 & $1.85 e-2$ \\
\hline 17 & $8.11 e-3$ \\
\hline 18 & $4.44 e-3$ \\
\hline 19 & $1.79 e-3$ \\
\hline 20 & $8.86 e-4$ \\
\hline 21 & $3.91 e-4$ \\
\hline 22 & $1.66 e-4$ \\
\hline 23 & $5.98 e-5$ \\
\hline 24 & $1.97 e-5$ \\
\hline 25 & $5.32 e-6$ \\
\hline 26 & $1.20 e-6$ \\
\hline 27 & $4.12 e-7$ \\
\hline 28 & $1.36 e-7$ \\
\hline 29 & $6.95 e-8$ \\
\hline 30 & $2.95 e-8$ \\
\hline 31 & $9.29 e-9$ \\
\hline 32 & $3.42 e-9$ \\
\hline 33 & $1.43 e-9$ \\
\hline 34 & $5.57 e-10$ \\
\hline 35 & $2.62 e-10$ \\
\hline 36 & $1.26 e-10$ \\
\hline 37 & $8.65 e-11$ \\
\hline
\end{tabular}

5.2.16. Ostrovsky-Hunter Equation. Ostrovsky-Hunter equation is

$$
\left(u_{t}-\beta u_{x x x}+\alpha u u_{x}\right)_{x}=\gamma u
$$

where $\alpha$ is a nonlinear coefficient and $\beta \in \mathbb{R}, \gamma>0$ are the dispersion coefficients. We assume a periodic boundary
TABLE 18: Maximum absolute error in the solution of the system of nonlinear equations for complex generalized Zakharov equation and varying $m$.

\begin{tabular}{lc}
\hline$m$ & $E$ \\
\hline 1 & $9.2329 e-1$ \\
2 & $7.0559 e-1$ \\
3 & $5.726 e-1$ \\
4 & $6.3466 e-2$ \\
5 & $4.3852 e-3$ \\
6 & $2.3221 e-4$ \\
7 & $1.5501 e-5$ \\
8 & $4.2396 e-7$ \\
9 & $2.8906 e-8$ \\
10 & $7.7742 e-10$ \\
11 & $8.6019 e-11$ \\
\hline
\end{tabular}

TABLE 19: Maximum absolute error in the solution of the system of nonlinear equations for Murray equation and varying $m$.

\begin{tabular}{lc}
\hline$m$ & $E$ \\
\hline 1 & $2.63 e-01$ \\
2 & $1.33 e-01$ \\
3 & $3.85 e-02$ \\
4 & $1.47 e-04$ \\
5 & $7.43 e-07$ \\
6 & $2.15 e-09$ \\
7 & $7.53 e-12$ \\
8 & $5.59 e-14$ \\
\hline
\end{tabular}

TABLE 20: Maximum absolute error in the solution of the system of nonlinear equations for nonlinear reaction and diffusion equation and varying $m$.

\begin{tabular}{lc}
\hline$m$ & $E$ \\
\hline 1 & $7.28 e-02$ \\
2 & $2.12 e-02$ \\
3 & $4.99 e-03$ \\
4 & $1.09 e-06$ \\
5 & $9.20 e-10$ \\
6 & $6.53 e-13$ \\
7 & $2.24 e-14$ \\
\hline
\end{tabular}

condition for this problem; that is,

$$
\begin{gathered}
u(0, t)=u(2 \pi, t), \\
u_{x}(0, t)=u_{x}(2 \pi, t), \\
u_{x x}(0, t)=u_{x x}(2 \pi, t), \\
u_{x x x}(0, t)=u_{x x x}(2 \pi, t) .
\end{gathered}
$$

The Chebyshev collocation method of first kind is used to discretize Ostrovsky-Hunter equation over a grid $30 \times 100$. The initial guess is $\mathbf{0}$ with initial and boundary conditions 
TABLE 21: Maximum absolute error in the solution of the system of nonlinear equations for reaction-diffusion equation with FischerKolmogorov reaction term and density dependent diffusion and varying $m$.

\begin{tabular}{lc}
\hline$m$ (1 Iter.) & $E$ \\
\hline 1 & $1.49 e+00$ \\
2 & $1.12 e+00$ \\
3 & $1.07 e+00$ \\
4 & $4.92 e-01$ \\
5 & $3.73 e-01$ \\
\hline$m(2$ Iter.) & $E$ \\
\hline 1 & $1.51 e-02$ \\
2 & $7.84 e-03$ \\
3 & $6.72 e-04$ \\
4 & $5.17 e-07$ \\
5 & $1.04 e-09$ \\
6 & $2.35 e-12$ \\
7 & $5.04 e-14$ \\
\hline
\end{tabular}

TABLE 22: Maximum absolute error in the solution of the system of nonlinear equations for two-dimensional nonlinear reactiondiffusion equation and varying $m$.

\begin{tabular}{lc}
\hline$m$ & $E$ \\
\hline 1 & $7.82 e-02$ \\
2 & $1.19 e-02$ \\
3 & $3.63 e-03$ \\
4 & $4.04 e-05$ \\
5 & $9.89 e-07$ \\
6 & $2.65 e-08$ \\
7 & $7.48 e-10$ \\
\hline
\end{tabular}

introduced. The residue is shown in Table 23. Our solution for the grid $\{0,6 \pi / 11, \pi, 16 \pi / 11\} \times\{0.1,0.2,0.3,0.4\}$ is given in Table 24. We notice that our results do not agree with those presented in [4]. Our solution is plotted in Figure 22.

\section{Conclusions}

Frozen Jacobian multistep iterative methods are computationally efficient to solve systems of nonlinear equations. This is because the LU factors of the Jacobian can be reused to solve additional linear systems at a very small additional cost. We have developed a new frozen Jacobian multistep iterative method with increased order of convergence for a given number of function evaluations. The claimed order of convergence of NMIM has been confirmed computationally by solving several small systems of nonlinear equations. NMIM has been used to solve fifteen nonlinear IVPs and BVPs with different collocation methods. As a whole, the Chebyshev collocation method of the first kind seems to provide the best numerical accuracy in the set of fifteen IVPs and BVPs considered in the paper.

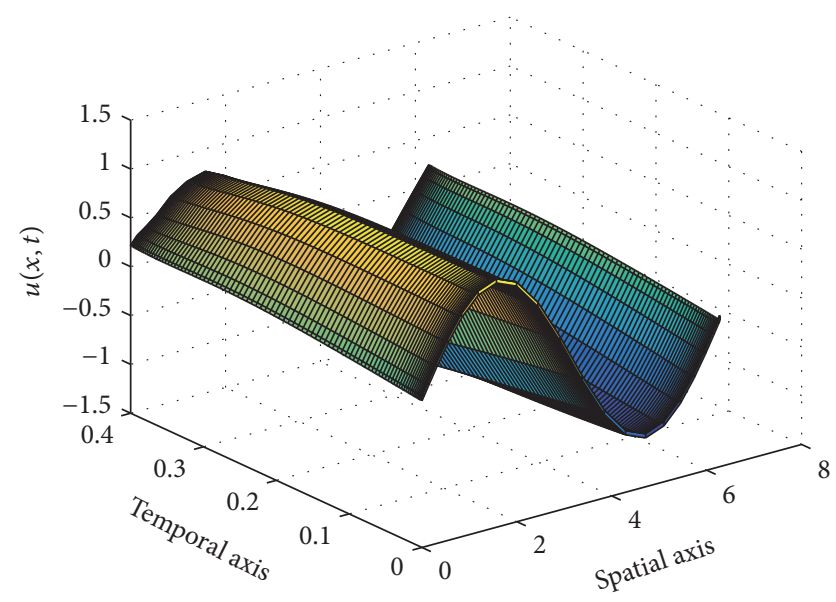

FIGURE 22: Solution for Ostrovsky-Hunter equation.

TABLE 23: Residue of the system of nonlinear equations for Ostrovsky-Hunter equation and varying $m$.

\begin{tabular}{lc}
\hline$m$ & $\|\mathbf{F}(\mathbf{x})\|_{\infty}$ \\
\hline 1 & 2 \\
2 & 2.5963 \\
3 & 2.797 \\
4 & 0.72034 \\
5 & 0.15165 \\
6 & 0.025873 \\
7 & 0.002996 \\
8 & 0.00020443 \\
9 & $8.7968 e-06$ \\
10 & $1.4609 e-07$ \\
11 & $1.3542 e-08$ \\
12 & $8.8853 e-10$ \\
13 & $8.5753 e-11$ \\
\hline
\end{tabular}

TABLE 24: Solution for Ostrovsky-Hunter equation.

\begin{tabular}{|c|c|c|c|c|}
\hline \multirow{2}{*}{$x$} & \multicolumn{4}{|c|}{$t$} \\
\hline & 0.1 & 0.2 & 0.3 & 0.4 \\
\hline 0 & 0.094587 & 0.17222 & 0.20233 & 0.21608 \\
\hline $6 \pi / 11$ & 0.96556 & 0.94696 & 0.8702 & 0.73399 \\
\hline$\pi$ & -0.10438 & -0.22921 & -0.41929 & -0.67358 \\
\hline $16 \pi / 11$ & -0.98195 & -0.96996 & -0.97341 & -0.97748 \\
\hline
\end{tabular}

\section{Competing Interests}

The authors declare that there is no conflict of interests regarding the publication of this paper.

\section{References}

[1] E. H. Doha, A. H. Bhrawy, M. A. Abdelkawy, and R. A. Van Gorder, "Jacobi-Gauss-Lobatto collocation method for the 
numerical solution of $1+1$ nonlinear Schrödinger equations," Journal of Computational Physics, vol. 261, pp. 244-255, 2014.

[2] A. H. Bhrawy, E. H. Doha, M. A. Abdelkawy, and R. A. Van Gorder, "Jacobi-Gauss-Lobatto collocation method for solving nonlinear reaction-diffusion equations subject to Dirichlet boundary conditions," Applied Mathematical Modelling. Simulation and Computation for Engineering and Environmental Systems, vol. 40, no. 3, pp. 1703-1716, 2016.

[3] A. H. Bhrawy, "An efficient Jacobi pseudospectral approximation for nonlinear complex generalized Zakharov system," Applied Mathematics and Computation, vol. 247, pp. 30-46, 2014.

[4] M. Dehghan and F. Fakhar-Izadi, "The spectral collocation method with three different bases for solving a nonlinear partial differential equation arising in modeling of nonlinear waves," Mathematical and Computer Modelling, vol. 53, no. 9-10, pp. 1865-1877, 2011.

[5] E. H. Doha, A. H. Bhrawy, and S. S. Ezz-Eldien, "Efficient Chebyshev spectral methods for solving multi-term fractional orders differential equations," Applied Mathematical Modelling. Simulation and Computation for Engineering and Environmental Systems, vol. 35, no. 12, pp. 5662-5672, 2011.

[6] E. H. Doha, A. H. Bhrawy, and R. M. Hafez, "On shifted Jacobi spectral method for high-order multi-point boundary value problems," Communications in Nonlinear Science and Numerical Simulation, vol. 17, no. 10, pp. 3802-3810, 2012.

[7] E. Tohidi and S. Lotfi Noghabi, "An efficient legendre pseudospectral method for solving nonlinear quasi bang-bang optimal control problems," Journal of Applied Mathematics, Statistics and Informatics, vol. 8, no. 2, pp. 73-85, 2012.

[8] G. Szego, Orthogonal Polynomials, Colloquium Publications XXIII, American Mathematical Society, 1939.

[9] J. Shen, T. Tang, and L.-L. Wang, Spectral Methods: Algorithms, Analysis and Applications, vol. 41, Springer, 2011.

[10] J. F. Traub, Iterative methods for the solution of equations, Prentice-Hall Series in Automatic Computation, Prentice-Hall, Englewood Cliffs, NJ, USA, 1964.

[11] J. M. Ortega and W. C. Rheinboldt, Iterative solution of nonlinear equations in several variables, Academic Press, New York, NY, USA, 1970.

[12] A. Cordero, M. Kansal, V. Kanwar, and J. R. Torregrosa, "A stable class of improved second-derivative free ChebyshevHalley type methods with optimal eighth order convergence," Numerical Algorithms, vol. 72, no. 4, pp. 937-958, 2016.

[13] V. Arroyo, A. Cordero, and J. R. Torregrosa, "Approximation of artificial satellites' preliminary orbits: the efficiency challenge," Mathematical and Computer Modelling, vol. 54, no. 7-8, pp. 1802-1807, 2011.

[14] D. A. Budzko, A. Cordero, and J. R. Torregrosa, "New family of iterative methods based on the Ermakov-Kalitkin scheme for solving nonlinear systems of equations," Computational Mathematics and Mathematical Physics, vol. 55, no. 12, pp. 19471959, 2015.

[15] S. Qasim, Z. Ali, F. Ahmad, S. Serra-Capizzano, M. Z. Ullah, and A. Mahmood, "Solving systems of nonlinear equations when the nonlinearity is expensive," Computers \& Mathematics with Applications, vol. 71, no. 7, pp. 1464-1478, 2016.

[16] U. Qasim, Z. Ali, F. Ahmad, S. Serra-Capizzano, M. Z. Ullah, and M. Asma, "Constructing frozen Jacobian iterative methods for solving systems of nonlinear equations, associated with ODEs and PDEs using the homotopy method," Algorithms (Basel), vol. 9, no. 1, 18 pages, 2016.
[17] F. Ahmad, E. Tohidi, and J. A. Carrasco, "A parameterized multi-step Newton method for solving systems of nonlinear equations," Numerical Algorithms, vol. 71, no. 3, pp. 631-653, 2016.

[18] M. Z. Ullah, S. Serra-Capizzano, and F. Ahmad, "An efficient multi-step iterative method for computing the numerical solution of systems of nonlinear equations associated with ODEs," Applied Mathematics and Computation, vol. 250, pp. 249-259, 2015.

[19] F. Ahmad, E. Tohidi, M. Z. Ullah, and J. A. Carrasco, "Higher order multi-step Jarratt-like method for solving systems of nonlinear equations: application to PDEs and ODEs," Computers \& Mathematics with Applications. An International Journal, vol. 70, no. 4, pp. 624-636, 2015.

[20] E. S. Alaidarous, M. Z. Ullah, F. Ahmad, and A. S. Al-Fhaid, "An efficient higher-order quasilinearization method for solving nonlinear BVPs," Journal of Applied Mathematics, vol. 2013, Article ID 259371, 11 pages, 2013.

[21] M. Z. Ullah, F. Soleymani, and A. S. Al-Fhaid, "Numerical solution of nonlinear systems by a general class of iterative methods with application to nonlinear PDEs," Numerical Algorithms, vol. 67, no. 1, pp. 223-242, 2014.

[22] H. Montazeri, F. Soleymani, S. Shateyi, and S. S. Motsa, "On a new method for computing the numerical solution of systems of nonlinear equations," Journal of Applied Mathematics, vol. 2012, Article ID 751975, 15 pages, 2012.

[23] F. Soleymani, T. Lotfi, and P. Bakhtiari, "A multi-step class of iterative methods for nonlinear systems," Optimization Letters, vol. 8, no. 3, pp. 1001-1015, 2014.

[24] S. Abbasbandy, E. Babolian, and M. Ashtiani, "Numerical solution of the generalized Zakharov equation by homotopy analysis method," Communications in Nonlinear Science and Numerical Simulation, vol. 14, no. 12, pp. 4114-4121, 2009.

[25] Q. S. Chang and H. Jiang, "A conservative difference scheme for the Zakharov equations," Journal of Computational Physics, vol. 113, no. 2, pp. 309-319, 1994.

[26] Q. S. Chang, B. L. Guo, and H. Jiang, "Finite difference method for generalized Zakharov equations," Mathematics of Computation, vol. 64, no. 210, pp. 537-553, 1995.

[27] M. Javidi and A. Golbabai, "Exact and numerical solitary wave solutions of generalized Zakharov equation by the variational iteration method," Chaos, Solitons and Fractals, vol. 36, no. 2, pp. 309-313, 2008.

[28] W. Bao, F. Sun, and G. W. Wei, "Numerical methods for the generalized Zakharov system," Journal of Computational Physics, vol. 190, no. 1, pp. 201-228, 2003.

[29] W. Bao and F. Sun, "Efficient and stable numerical methods for the generalized and vector Zakharov system," SIAM Journal on Scientific Computing, vol. 26, no. 3, pp. 1057-1088, 2005. 


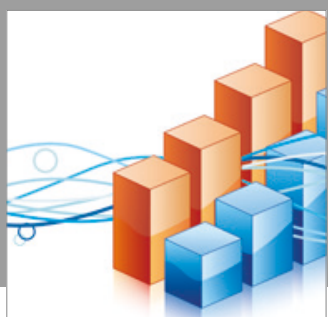

Advances in

Operations Research

vatersals

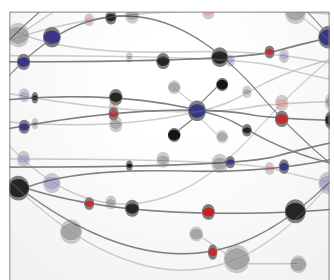

\section{The Scientific} World Journal
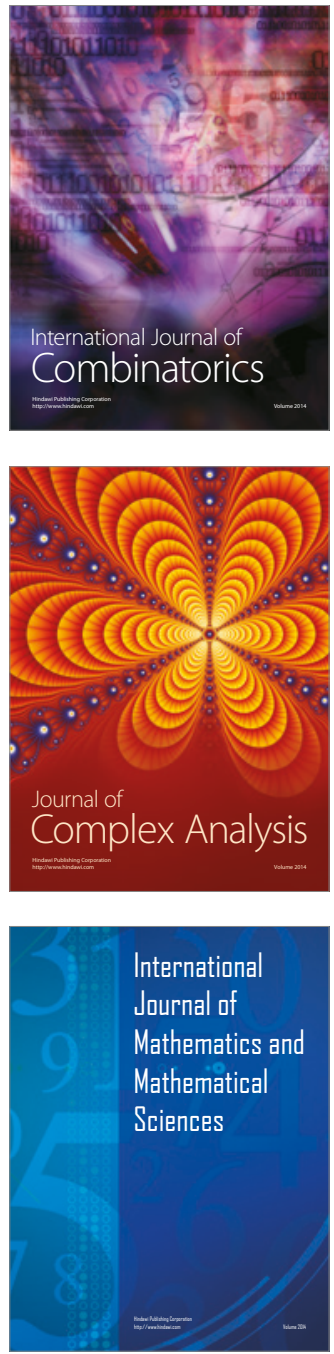
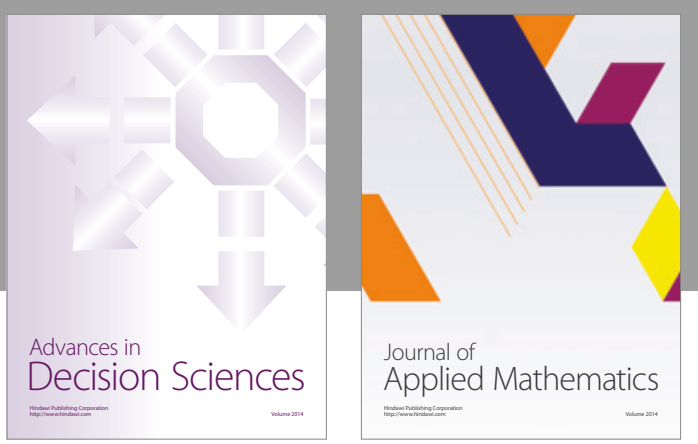

Algebra

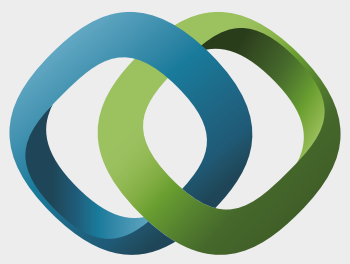

\section{Hindawi}

Submit your manuscripts at

https://www.hindawi.com
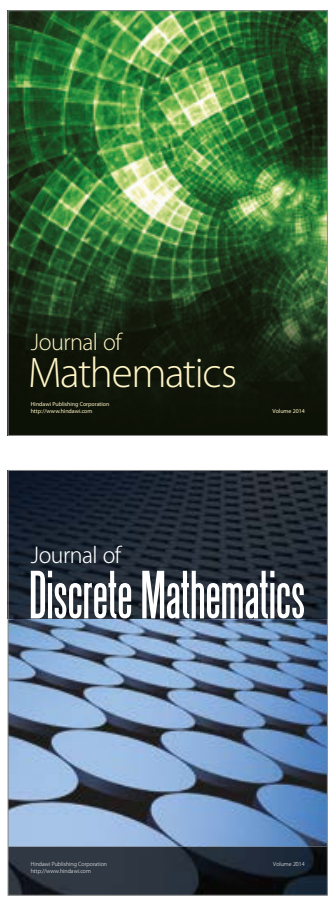

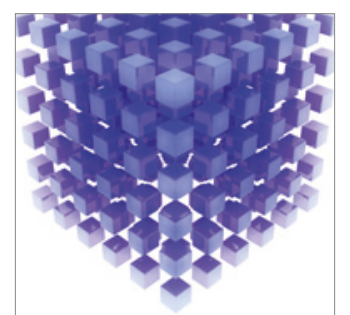

Mathematical Problems in Engineering
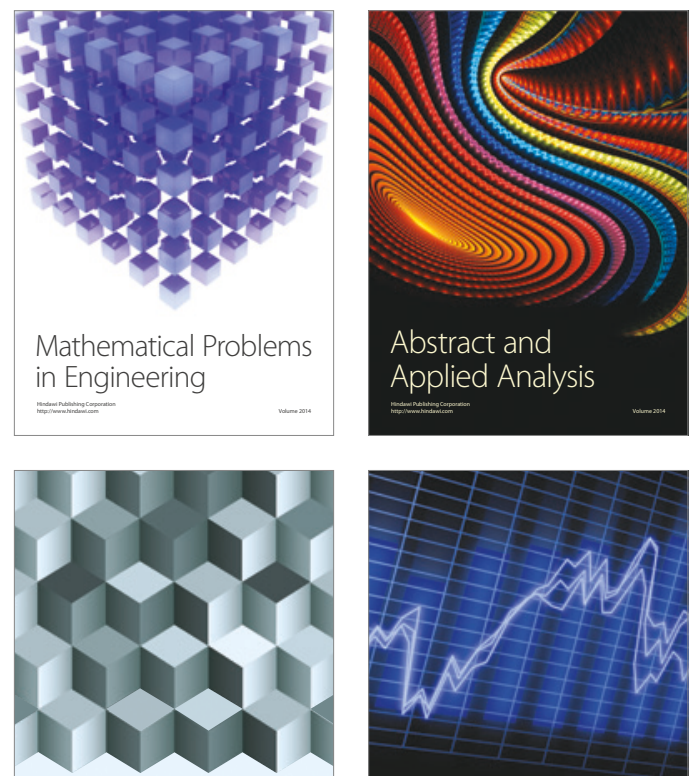

Journal of

Function Spaces

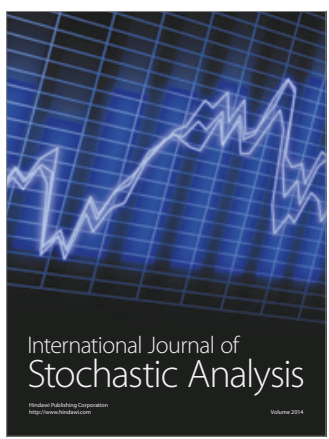

Probability and Statistics
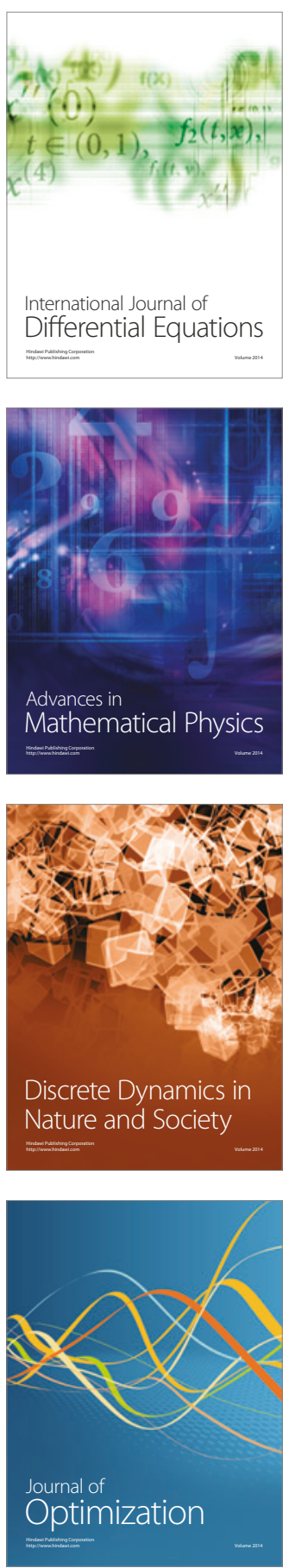